\title{
The interplay between curvature and composition in binary mixture lipid bilayers
}

\section{Dissertation}

\author{
for the award of the degree \\ "Doctor of Philosophy" Ph.D. Division of Mathematics \\ and Natural Sciences \\ of the Georg-August-Universität Göttingen \\ within the doctoral program PROPHYS \\ of the Georg-August University School of Science
}

Submitted by

\section{Israel Abraham Barragán Vidal}

from Distrito Federal, Mexico

Göttingen, 2016 


\section{Thesis Committee}

Marcus Müller Institut für Theoretische Physik, Georg-AugustUniversität

Annette Zippelius Institut für Theoretische Physik, Georg-AugustUniversität

\section{Members of the Examination Board}

Referee: Marcus Müller, Institut für Theoretische Physik, Georg-August-Universität

Co-Referee: Annette Zippelius, Institut für Theoretische Physik, Georg-August-Universität

Other Members: Jörg Enderlein, III Physikalisches Institut, GeorgAugust-Universität

Tim Salditt, Institut für Röntgenphysik, GeorgAugust-Universität

Stefan Klumpp, Institut für Nichtlineare Dynamik, Georg-August-Universität

Reiner Kree, Institut für Theoretische Physik, Georg-August-Universität

Date of the oral examination: 9.02.2016 


\section{Acknowledgments}

In the first place, I want to express my gratitude to Professor Marcus Müller, for giving me the opportunity of being part of his group and for his invaluable guidance for the realization of this work. I would also like to thank him for his patience, motivation and immense knowledge, but more than that, for being always disposed to explain things in a clear and concise way.

Beside my advisor, I want to thank other members of the group and the faculty for their support and company during these years.

Financial support by the German Science Foundation within the SFB 937 "Collective behavior of soft and biological matter" TP A7, the German Academic Exchange Service (DAAD) and the National Council of Science and Technology (CONACyT) grants are gratefully acknowledged. Computing in the HLRN Hannover/Berlin and the JSC Jülich, Germany is also gratefully acknowledged.

Finally I want to thank my parents, my sister, and Maria for always being there. 


\section{Abstract}

In this work we investigate, via computer simulations with an implicit-solvent coarse-grained model, the interplay between curvature and composition in selfassembled lipid structures comprised by two lipid species with different spontaneous curvatures. The different structures considered in this study are: i) planar bilayers with thermally induced shape fluctuations, which allow the investigation of low curvature regimes, ii) cylindrical bilayers with different midplane radius of curvature and iii) inverted-monolayer tubes immersed in an hydrophobic solvent. Beside these different self-assembled morphologies and curvature regimes, this study also considers different degrees of segregation between unlike lipid species.

To analyze the results from simulations with planar and cylindrical bilayers, a phenomenological model describing the thermodynamics of mixing in the two monolayers of lipid membranes was proposed. The novelty of this model is that beside incorporating contributions from the free energy of mixing on the two membrane leaflets, it also accounts for their difference in curvature. The later contribution becomes particularly important for membranes whose local curvature modulations are comparable to the inverse bilayer thickness. A salient prediction of the model is the existence of a saturation curvature for which the composition difference between the apposing leaflets becomes maximal. The occurrence of this saturation curvature will depend on system segregation. In particular, for low incompatibility between lipid species, the saturation curvature may lie beyond the inverse bilayer thickness, which sets a limit on the curvatures that are physically accessible to the system. In contrast, for high incompatibility the saturation curvature may be comparable to the thermally-induced curvature fluctuations observed in planar bilayers.

Another advantage of the proposed model is that many of its parameters can be directly measured from easily accessible experimental quantities, such as the power spectra of shape and composition fluctuations. Furthermore, the two parameters to fit from measurements of the local curvature and composition asymmetry across the bilayer, are directly related to the intrinsic curvature of the constituting lipids. 
Finally, spontaneous curvatures from the analysis with bilayer membranes were in good agreement with those obtained from a common experimental technique, where lipids with unknown curvature are inserted into an inverted structure whose self-assembled and spontaneous curvatures coincide. It has been seen, however, that this technique may only be used under low segregation conditions and that the anisotropy of this inverted structures together with the change of lipid conformations due to the interdigitation of the hydrophobic solvent inside of the tail region may induce errors in the determination of the spontaneous curvature of the guest lipids. 


\section{Kurzfassung}

Mittels Computersimulationen eines vergröberten Membranmodells mit implizitem Lösungsmittel habe ich in dieser Arbeit das Wechselspiel zwischen Krümmung und Zusammensetzung in selbst-assemblierten Lipid-Strukturen, welche aus zwei Lipidarten mit unterschiedlichen spontanen Krümmungen bestehen, untersucht. Verschiedene Strukturen wurden betrachtet: i) plane Doppelschichten mit thermischen Undulationsfluktuationen, welche es erlauben den Bereich schwacher Krümmung zu studieren, ii) zylindrische Membranröhren mit unterschiedlicher Krümmung, sowie iii) invertierte wurm-artige Mizellen (Röhren von Einzelschichten) in einem hydrophoben Lösungsmittel. Zusätzlich zu diesen unterschiedlichen, selbst-assemblierten Strukturen betrachtet diese Studie auch verschiedene Unverträglichkeiten zwischen den beiden Lipidarten.

Zur Analyse der Simulationsdaten planer Doppelschichten und Membranröhren schlage ich ein phänomenologisches Modell vor, welches die Thermodynamik der Lipidmischung in den beiden Monolagen der Membran erfasst. Die Neuheit dieses Models besteht darin, dass es neben der freien Energie der Mischung in den beiden Monolagen auch deren Krümmungsunterschied berücksichtigt. Letzter Beitrag wird besonders wichtig, wenn die lokale Membrankrümmung mit der inversen Doppelschichtdicke vergleichbar wird. Eine besondere Vorhersage des Modells ist die Existenz einer Sättigungskrümmung, bei welcher der Zusammensetzungsunterschied zwischen den beiden, gegenüberliegenden Monolagen maximal wird. Das Auftreten dieser Sättigungskrümmung hängt von der Unverträglichkeit der Lipidarten ab: Für niedrige Unverträglichkeit kann die Sättigungskrümmung über der inversen Membrandicke, welche die maximale, physikalisch erreichbare Krümmung kennzeichnet, liegen. Im entgegengesetzten Fall starker Unverträglichkeit kann die Sättigungskrümmung vergleichbar mit der durch thermische Fluktuationen induzieren lokalen Membrankrümmung werden.

Ein weiterer Vorteil des vorgeschlagenen Modells besteht darin, dass viele Modellparameter unmittelbar durch einfach zugängliche experimentelle Kenngrößen -wie etwa die Potenzspektren von Form- und Zusammensetzungsfluktuationen -messbar sind. Die weiteren zwei anpassbaren Modellparameter, wel- 
che die gemessenen Asymmetrien der lokalen Krümmung und Zusammensetzung zwischen den Monolagen quantifizieren, stehen in unmittelbarem Zusammenhang zu den intrinsischen spontanen Krümmungen der beiden Lipidarten.

Die spontanen Krümmungen, welche aus der Analyse der Doppelschichten erzielt wurden, stimmen gut mit denen, welche aus einer gebräuchlichen experimentellen Technik bestimmt wurden, überein. Dabei werden Lipide mit einer unbekannten spontanen Krümmung (als Gäste) zu einer invertierten Struktur hinzugefügt, bei der die spontane Krümmung der Einzelschicht mit der Krümmung der inversen Struktur übereinstimmt. Meine Simulationen zeigen jedoch, dass diese Technik nur für schwach unverträgliche Mischungen funktioniert und dass die Anisotropie der invertierten Struktur und die Änderung der Lipidkonformationen durch das Eindringen des hydrophoben Lösungsmittel in die Schwanzregion zu Fehlern in der Bestimmung der spontanen Krümmung des Gastlipids führen kann. 


\section{Contents}

$\begin{array}{lll}1 & \text { Introduction } & 1\end{array}$

1.1 Plasma membrane . . . . . . . . . . . . . . . . . . . . . 1

1.1 .1 Lipid self-assembly . . . . . . . . . . . . . . . . . . 3

1.1 .2 Bilayer heterogeneity $\ldots \ldots \ldots \ldots \ldots$

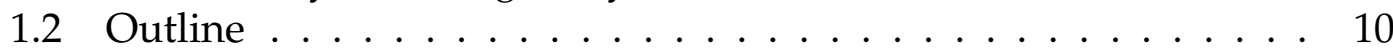

2 Model 13

2.1 Coarse-Graining . . . . . . . . . . . . . . . . . . . 13

2.2 Coarse-grained, solvent free model . . . . . . . . . . . . . . 17

2.2 .1 Bonded interactions . . . . . . . . . . . . . . . . 17

2.2 .2 Non-bonded interactions . . . . . . . . . . . . . . 18

2.3 Numerical implementation . . . . . . . . . . . . . . . . 25

2.3 .1 Model discretization . . . . . . . . . . . . . . . . 25

2.3 .2 Simulation ensembles . . . . . . . . . . . . . 26

2.4 Parallelization . . . . . . . . . . . . . . . . 30

3 Phenomenological membrane models 37

3.1 Early membrane models $\ldots \ldots \ldots \ldots$. . . . . . . . . . . . 38

3.1 .1 Elastic models . . . . . . . . . . . . . . . . . . . . 38

$3.1 .2 \quad$ Beyond purely elastic models . . . . . . . . . . . . . . 41

3.2 Composition-dependent free energy of mixing . . . . . . . . . 44

3.3 Curvature-dependent bending rigidity $\ldots \ldots \ldots \ldots$. . . . . 54

$4 \quad$ Interplay between curvature and composition in lipid bilayers $\quad 57$

4.1 Simulation setup $\ldots \ldots \ldots \ldots \ldots \ldots \ldots \ldots \ldots$

$4.1 .1 \quad$ Area per lipid . . . . . . . . . . . . . . . . . . . . 60

$4.1 .2 \quad$ Area compressibility . . . . . . . . . . . . . . . . . . 61

4.1 .3 Lateral pressure profile . . . . . . . . . . . . . . . 62

4.2 Non-free parameters for the phenomenological model . . . . . . . 64

4.2 .1 Susceptibility of composition fluctuations . . . . . . . . . . 64

4.2 .2 Density profile $\ldots \ldots \ldots \ldots \ldots \ldots$ 
$4.2 .3 \quad$ Power spectra of height fluctuations . . . . . . . . . . . . 71

4.2 .4 Summary . . . . . . . . . . . . . . . 73

4.3 Effective curvature-composition coupling . . . . . . . . . . . 74

4.3.1 Joint probability distribution for $\phi$ and $\mathrm{H} \ldots \ldots . . . . .74$

4.4 Cylindrical bilayers . . . . . . . . . . . . . . . 79

5 Spontaneous curvature from inverted lipid structures 83

5.1 Self-assembly of inverted structures $\ldots \ldots \ldots$. . . . . . . . . . . 83

5.2 Prevention of void-induced stretching . . . . . . . . . . . . . 86

5.3 Simulation of inverted cylinders $\ldots \ldots \ldots$. . . . . . . . 88

\begin{tabular}{lll}
\hline & Summary and outlook & 93
\end{tabular}

\begin{tabular}{lr}
\hline Bibliography & 99
\end{tabular} 


\section{List of Figures}

1.1 Schematic representation of the plasma membrane . . . . . . . . 2

1.2 Membrane lipids . . . . . . . . . . . . . . . . . . . . 4

1.3 Self-assembled structures . . . . . . . . . . . . . . . . 5

1.4 Lipid rafts . . . . . . . . . . . . . . . . 6

1.5 Liquid-order and liquid-disorder coexistence . . . . . . . . . . . 7

1.6 Composition asymmetry of lipid bilayers . . . . . . . . . . . . . . . . 8

1.7 Mixed and sorted bilayers $\ldots \ldots \ldots$. . . . . . . . . . . . . . . . .

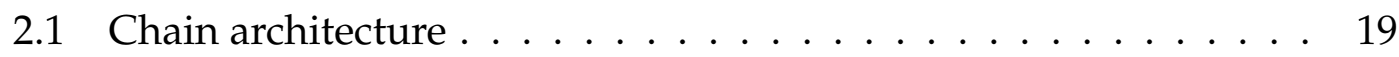

2.2 Equation of state for pure hydrophobic/hydrophilic substances . 21

2.3 Weighting functions for non-bonded interactions . . . . . . . . . . 23

2.4 Semi-grand canonical mutation . . . . . . . . . . . . . . . . . . . . . . . . . . . . . 39

2.5 Domain decomposition . . . . . . . . . . . . . . . . . 31

2.6 Domain decomposition algorithm for NVT and $\mathrm{N} \Delta \mu \mathrm{T}$ ensembles. 34

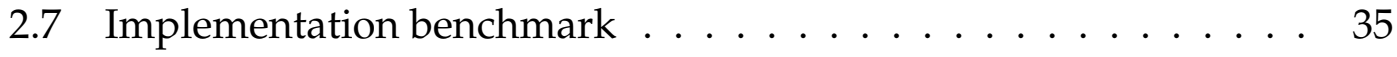

3.1 Monge parametrization . . . . . . . . . . . . . . . . . . . . . 40

3.2 Curved membrane patch . . . . . . . . . . . . . . . . . 46

3.3 Composition domain in the single- and joint-monolayers repre-

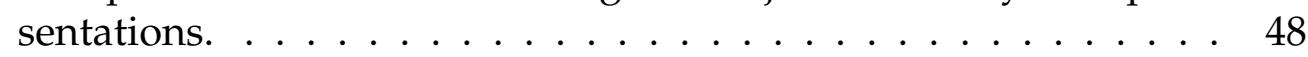

3.4 Implicit relation between composition difference and curvature . 51

3.5 Assembly of lipids with commensurate and incommensurate cur-

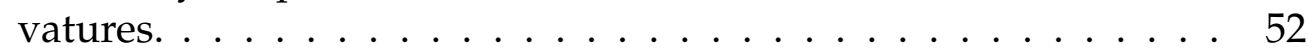

$\begin{array}{lll}3.6 & \text { Effective coupling between composition difference and curvature } 54\end{array}$

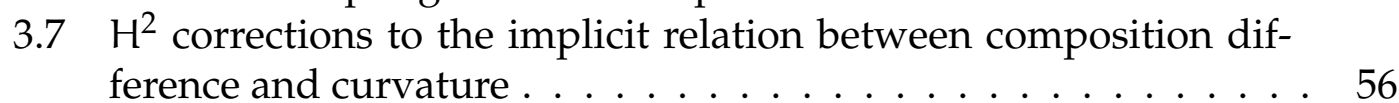

4.1 Self-assembly into planar bilayers . . . . . . . . . . . . . . . 60

4.2 Pressure profile $\ldots \ldots \ldots \ldots$. . . . . . . . . . . . . 63

4.3 Average composition as a function of the difference in chemical

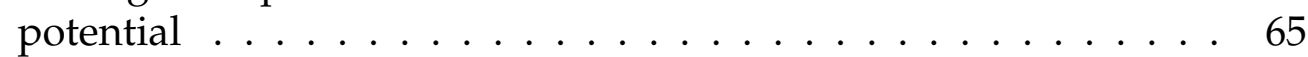


4.4 Susceptibility of composition fluctuations . . . . . . . . . . . . 67

4.5 Density profile . . . . . . . . . . . . . . . . . . 69

4.6 Spectra of height fluctuations . . . . . . . . . . . . . . 72

4.7 Grid mappings $\ldots \ldots \ldots \ldots$. . . . . . . . . . . . . . . 75

4.8 Joint probability distribution for curvature and composition dif-

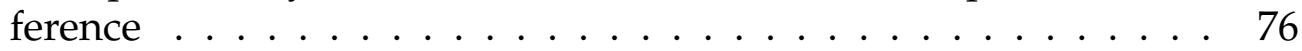

4.9 Effective curvature-composition coupling . . . . . . . . . . . 77

4.10 Comparison of the lateral sorting of lipid for low and high in-

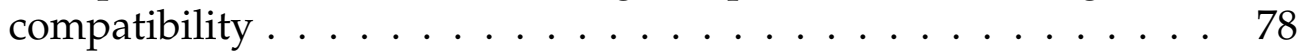

4.11 Cylinders . . . . . . . . . . . . . . . . . . . . 80

4.12 Comparison between results from planar and cylindrical configurations and the predictions from the mean-field model . . . . . 81

5.1 Lipid inverted phases . . . . . . . . . . . . . . . . . . 84

5.2 Anisotropic deformations of inverted hexagonal phase . . . . . . 85

5.3 Voids in the inverted hexagonal phase . . . . . . . . . . . . 85

5.4 Inverted monolayer immersed in an hydrophobic melt . . . . . . 87

5.5 Snapshots of the simulations with inverted cylinders . . . . . . . 89

5.6 Inverted cylinder radius vs height $\ldots \ldots$. . . . . . . . . . . . . . . . . 90

5.7 Parallel pressure vs cylinder length . . . . . . . . . . . . . . . . . . 91

6.1 Diffusion on mixed bilayer tubes . . . . . . . . . . . . . . . 98 


\section{List of Tables}

4.1 Self-assembled morphologies obtained from a random initial con-

\begin{tabular}{|c|c|}
\hline & figuration. The column headers on the right denote the molecu- \\
\hline & lar asymmetry, e.g., $10 / 6$ means $\mathrm{N}_{\mathrm{A}}=10$ and $\mathrm{N}_{\mathrm{B}}=6$. The mor- \\
\hline & phologies are abbreviated: $\mathrm{s}=$ spherical micelles, $\mathrm{c}=$ cylindrical mi- \\
\hline & celles, $\mathrm{w}=$ wormlike micelles, $\mathrm{b}=$ bilayers, $\mathrm{i}=$ inverted structures, \\
\hline & i.e., bilayers with hydrophilic inclusions. . . . \\
\hline 4.2 & Set of parameters used in this work for the study of the curvature- \\
\hline & composition coupling, $\Lambda_{\text {eff. }}$ \\
\hline 4.3 & Summary of bilayer properties used in the curvature-composition \\
\hline & coupling analysis. \\
\hline
\end{tabular}

5.1 Set of parameters obtained from the fits of Eq. 5.7|and Eq. $5.5 \mid$ to the \begin{tabular}{l|l} 
cylinder radius and parallel pressure measured from simulations. & 92
\end{tabular} 


\section{Chapter 1}

\section{Introduction}

\subsection{Plasma membrane}

One of the first approaches to explain the difference between living organisms and inorganic matter, relied on the existence of a vital spirit governing the processes of organic entities. Drawbacks to this approach first appeared when chemists successfully synthesized organic compounds from inorganic materials. ${ }^{1]}$ Since then, it has been shown that, although different in the complexity of their structure, both kinds of compounds are ruled by the same microscopic physical laws. However, knowing the properties of the these building blocks at a molecular level does not provide much information on the behavior of an entire cell, where a plethora of proteins, lipids and nucleic acids merge together and give rise to a new set of properties and collective phenomena. Examples of these are: the self-ensemble of phospholipids into complex structures like micelles, liposomes or bilayers, solute transport through ion channels, cellular movement and cell adhesion.

Until recently, these complex structures and processes were understood in a descriptive way, only in the context of biological sciences. To achieve a quantitative understanding of the fundamental laws ruling their behavior it is necessary to apply some methods and tools developed in a physics approach. In this context, particularly important are the tools used in soft and condensed matter physics to study the physical properties of objects (e.g., atoms, molecules, grains of sand, or soap bubbles) placed in sufficiently close proximity to each other, so that interactions between them cannot be ignored.

During the last decades an increasing amount of research has been focused on the physical mechanisms behind biological processes, such as the complex biochemical reactions taking place in lipid membranes. These membranes are molecular aggregates present in all kinds of living matter. Together with pro- 


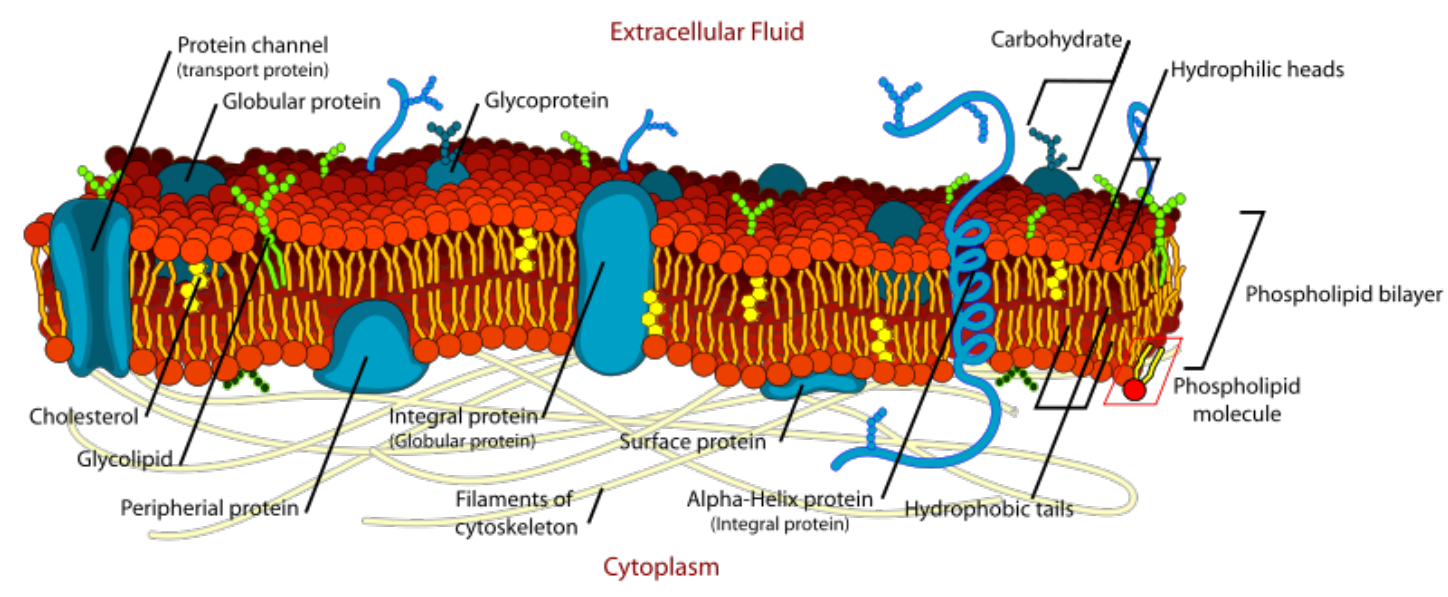

Figure 1.1: Schematic representation of the plasma membrane. Image by Mariana Ruiz Villareal.

teins, they constitute the plasma membrane surrounding the cell and many of its organelles, such as mitochondria, the Golgi apparatus and even the envelope of some virus species. A schematic representation of the plasma membrane is shown in Fig $1.1{ }^{2}$ Beside working as a barrier, isolating these cellular structures from their environment and defining their shape, the selective permeability of the plasma membrane plays an active role in the regulation of the processes taking place therein. ${ }^{\sqrt[34]{4}}$ Likewise, proteins embedded into the extracellular membrane regulate the transport of nutrients in and out of the cell. Furthermore, the presence of transmembrane receptors confer cells the ability to recognize signals from the exterior and activate specific processes in response. Examples of these include the production of specific proteins, the contraction of muscle cells and cellular division. 516

The role of the lipid membrane in these regulatory mechanisms comes about via lipid-protein interactions. Recent experimental evidence suggests that the expression of certain protein and antigen activities is only possible in the presence of specific phospholipids. ${ }^{7}$ This hypothesis is supported by the observed compartmentalization of proteins within composition inhomogeneities of the lipid membrane (also known as lipid rafts). ${ }^{8}$ These highly dynamic and transient structures are characterized by a sub-second time scale and their typical sizes are in the range of $10-200 \mathrm{~nm}$. The origin of these rafts, however, is still under debate. Possible mechanisms to explain their formation include:

- The limited spatial extent of rafts is related to the large but finite correlation length of composition fluctuations in the ultimate vicinity of the critical point of the multicomponent lipid membranes. ${ }^{9}$ 
- Ultra-low line tension due to the presence of lineactants in conjunction with thermal fluctuations results in a microemulsion-like structure. 1011

- Quenched disorder due to interactions with proteins of the cytoskeleton prevents macroscopic phase separation. ${ }^{12113}$

- The curvature-composition coupling imparts a microemulsion-like structure on the mixture with a characteristic length scale dictated by the elastic properties of the membrane. 14

In this work I devise computer simulation techniques that support this last hypothesis. Specifically, I investigate the correlation between the local curvature and composition in binary mixture membranes and quantify the spontaneous curvature of the individual lipid species. Furthermore, I point out that besides raft-formation, the coupling between curvature and composition may account for packing effects that are particularly important on highly-curved structures, such as membrane tubes or transition states that occur in transformation of membrane shapes (e.g., pore formation, fusion and fission).

\subsubsection{Lipid self-assembly}

The characteristic feature of membrane forming lipids is their amphiphilic nature. These lipids comprise an hydrophilic head-group, which favors the interaction with water and other polar substances, and an hydrophobic group, consisting of one or two long hydrocarbon chains, which can be saturated or unsaturated. Being non-polar, these hydrocarbon tails are typically confined into dense liquid regions when suspended in water. Schematics of lipids that self-assemble into bilayer membranes are shown in Fig 1.2 . It is the presence of these two opposite interactions with water, within a single molecule, what makes lipids an essential component for the formation of thin, albeit resistant and flexible, enclosures of individual entities, such as cells and many of their organelles. $\frac{15-18}{1-18}$

Besides the lipid-water and lipid-lipid interactions inherent to the amphiphilic architecture, the self-assembly of lipids into different morphologies is also dictated by their geometry. This can be reasoned in the following heuristics: starting from very diluted conditions, increasing the concentration of lipids in solution, will eventually result in the formation of an oil-water interface. Once this stage has been reached, further increments of the lipids concentration will result in reductions of the projected area per lipid. The reason for this is to minimize the unfavorable contact between the hydrophobic region and the solvent, thus resulting in an effective attraction of molecules at the interface. ${ }^{2021}$ This attraction will be characterized by a prominent negative peak in the profile of the 


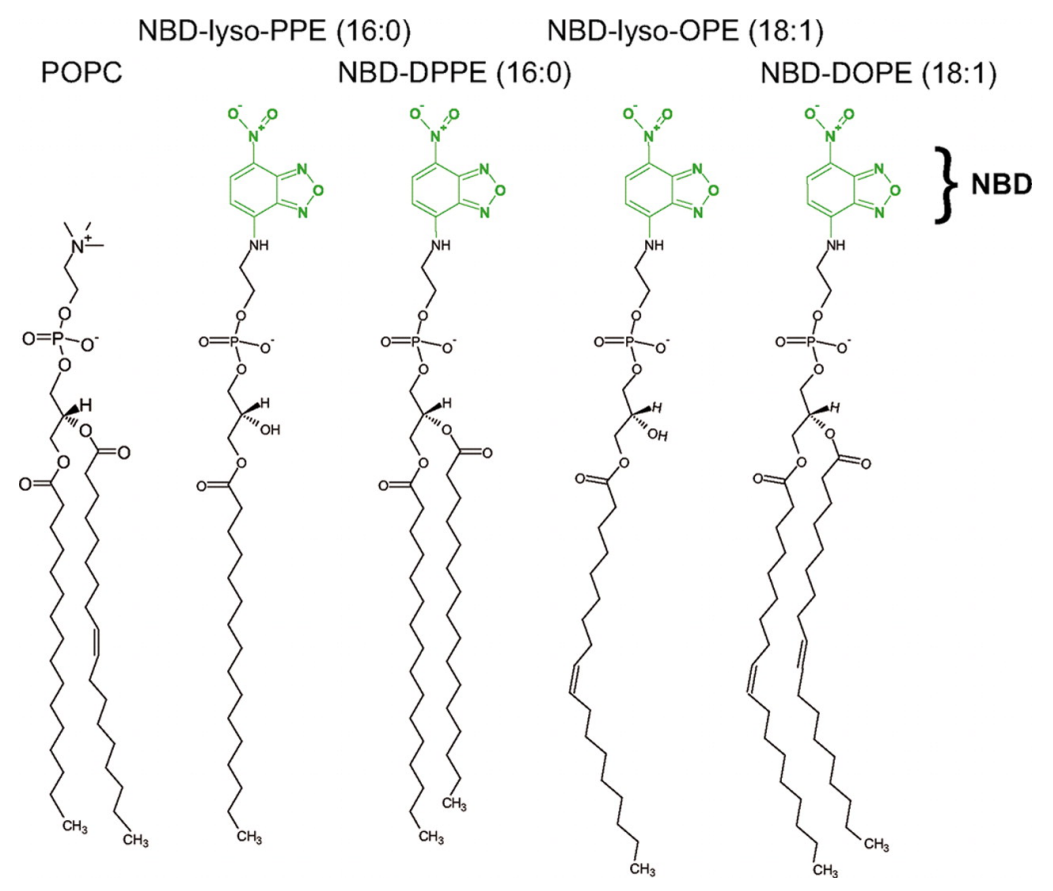

Figure 1.2: Schematic representation of common membrane lipids. Image taken from Kamala et al. [19].

pressure distribution, perpendicular to the interface. ${ }^{2223}$ At some point, however, the incompressibility conditions of the hydrophobic liquid will be met. This equilibrium state will be dictated by the force balance between the attraction at the interface and the steric repulsion between the tightly packed, yet highly-mobile lipid tails. Once this equilibrium state has been reached, both the area of the hydrophilic head-groups, $\mathrm{a}_{0}$, and the volume, $v_{0}$, of the hydrophobic tails will remain constant and further concentration increments will result in global deformations of the self assembled structure that satisfy the incompressibility requirements. The optimal values of $a_{0}$ and $v_{0}$, together with the critical tail length, $l_{0}$, (which sets a limit in the length of the chains) provide a crude, first estimation of the geometrical shape of the individual lipids and their final aggregates. With these quantities, it is possible to define the dimensionless packing parameter ${ }^{24}$

$$
\alpha=\frac{v_{0}}{\mathrm{a}_{0} \mathrm{l}_{0}} .
$$

This parameter quantifies the ratio of the tail volume to the volume projected by the optimal head-group area. Therefore, it can be thought of as a measure of the intrinsic or spontaneous lipid curvature (that is, the curvature in absence of external constraints or unbalanced torsional forces). For example, to self- 

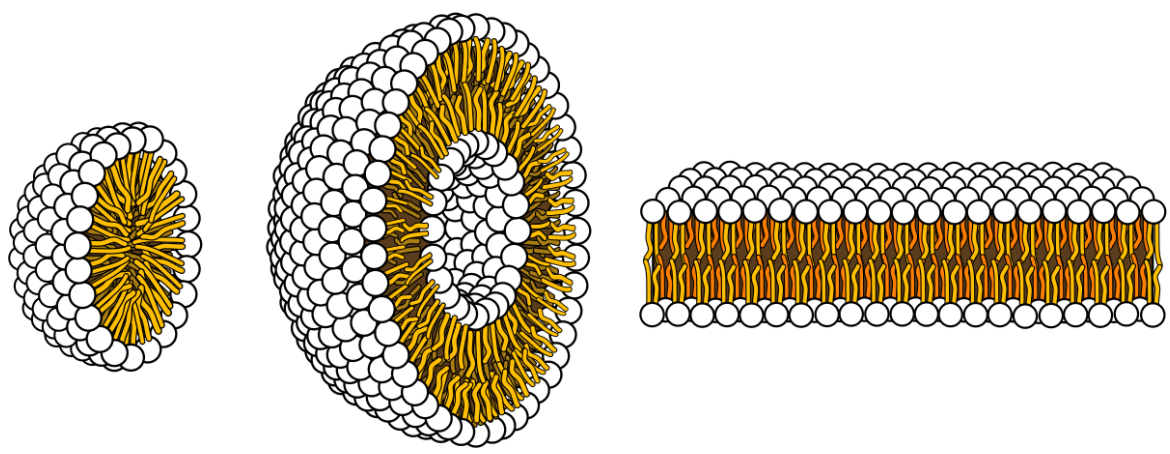

Figure 1.3: From left to right, schematic representation of self-assembled spherical micelles, vesicles and planar bilayers. Image by Mariana Ruiz Villareal.

assemble $\mathrm{N}$ lipids into a spherical micelle of total volume $\mathrm{V}$ and surface area $A$, the ratio between the current, $a_{c}$, and the optimal, $a_{0}$, area per lipid is given by

$$
\frac{\mathrm{a}_{\mathrm{c}}}{\mathrm{a}_{0}}=3 \frac{v_{0}}{\mathrm{ra}_{0}}=3 \frac{\mathrm{l}_{0}}{\mathrm{r}} \alpha,
$$

where $r$ is the micelle radius. Since $r$ is bounded by $l_{0}$, this expression implies that as long as the the packing parameter is smaller than $1 / 3$, it is possible to self-assemble the hypothesized cone-like lipids, into spherical micelles (leftmost schematics in Fig, 1.3). In the same manner, wedge-like lipids with packing parameters $1 / 3<\alpha<1 / 2$ will self-assemble into cylindrical micelles. Lipid architectures with packing parameters between $1 / 2$ and 1 will result in vesicles and planar bilayers, whereas architectures with $\alpha>1$ will no longer be soluble in water and at high concentrations will organize into structures with liquid crystalline order, such as the inverted hexagonal or inverted cubic phases.

\subsubsection{Bilayer heterogeneity}

Biological membranes typically comprise a mixture of hundreds of different lipid species. Although the packing parameter of most of these lipids is close to 1 , there are remarkable examples where deviations from the cylindrical geometry are considerable. Sphingolipids, for instance, are an entire family of membrane lipids with cone-like geometry and whose packing parameters can be as low as $\alpha \sim 0.4$. These lipids are usually found in the extracellular monolayer of mammalian cells, in mol fractions between $10-20 \%$. When inserted into an homogeneous flat bilayer, these lipids will induce a torque on each monolayer $\left(\mathrm{T} \sim \int \Pi z \mathrm{~d} z\right.$, with $\Pi$ the pressure profile across the bilayer). When torques remain unbalanced, their net effect is to bend the individual monolayers along their normal direction. If these "wrong" lipids are distributed symmetrically in 


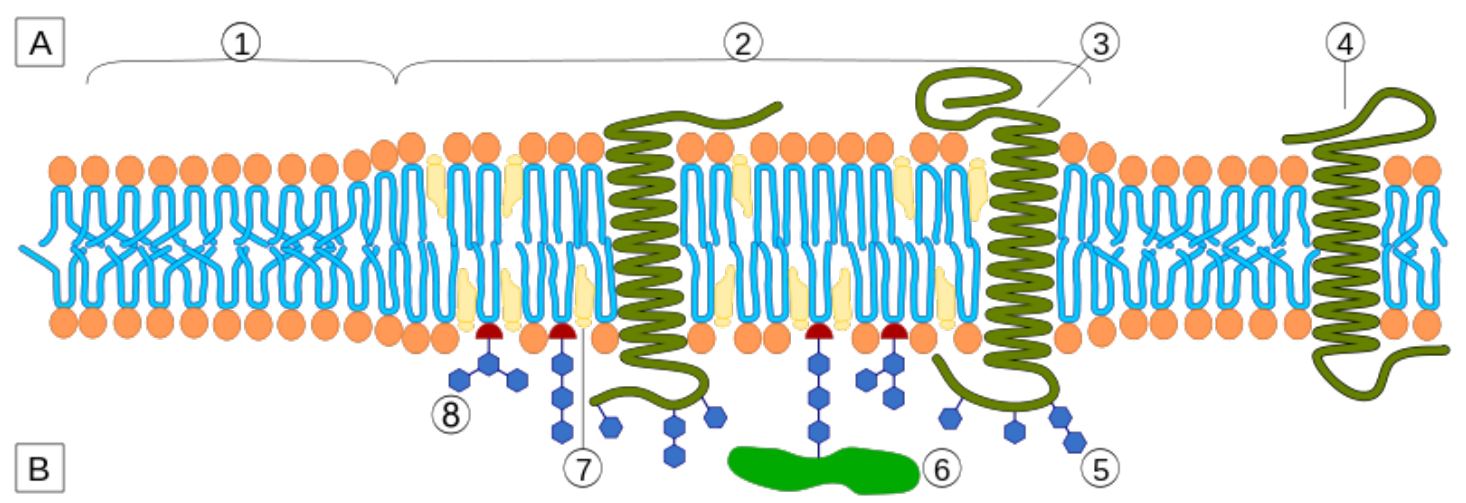

Figure 1.4: Ordered-disordered lipid rafts induced by the shape readjustment of non-cylindrical lipids. Labels denote the following: A) intracellular space or cytosol, B) extracellular space or vesicle/Golgi apparatus lumen, 1) non-raft membrane, 2) lipid raft, 3) lipid raft associated transmembrane protein, 4) monraft membrane protein, 5) glycosylation modifications (on glycoproteins and glycolipids), 6) GPI-anchored protein, 7) cholesterol and 8) glycolipid. Image by Artur Jan Fijalkowski.

both monolayers, the torques will act in opposite directions, therefore attempting to tear the bilayer apart. Creating a void region within the hydrophobic interior, however, would be a very energetically unfavorable process. ${ }^{[25}$ Therefore, up to a critical concentration, the inserted sphingolipids will have to reshape in order to fit into the matrix of cylindrical lipids. This shape readjustment will result in a macroscopic elastic stress stored in the flat bilayer. ${ }^{26}$

Shape deformations induced by the inclusion of non-cylindrical lipids into stress-free, flat bilayers, will be constrained by the incompressibility condition of the hydrophobic interior: if the hydrophobic volume per lipid has to remain constant, the transformation of cone-shaped lipids into cylindrical ones will induce (in general) an overall compression of the tails cross-sectional area and a concomitant stretching along the axial direction. Such deformations will come along with a drastic reduction of the tail configurational entropy, therefore promoting the emergence of regions where the lipid tails are highly order along the membrane normal (see region 2 of Fig,1.4). The lateral diffusion of these elongated lipids, however, is still comparable to that of the cylindrical (unstretched) lipids in the fluid-disordered state (denoted as $\mathrm{L}_{\alpha}$ ). ${ }^{2728}$ For this, this compression-induced lipid arrangement is usually named a liquid-ordered phase $\left(\mathrm{L}_{\beta}\right)$.

In recent years, the coexistence of these ordered and disordered phases has been extensively investigated in ternary model systems. 19129-31] Usually, these systems comprise a mixture of sphingolipids and cholesterol (which has a small 

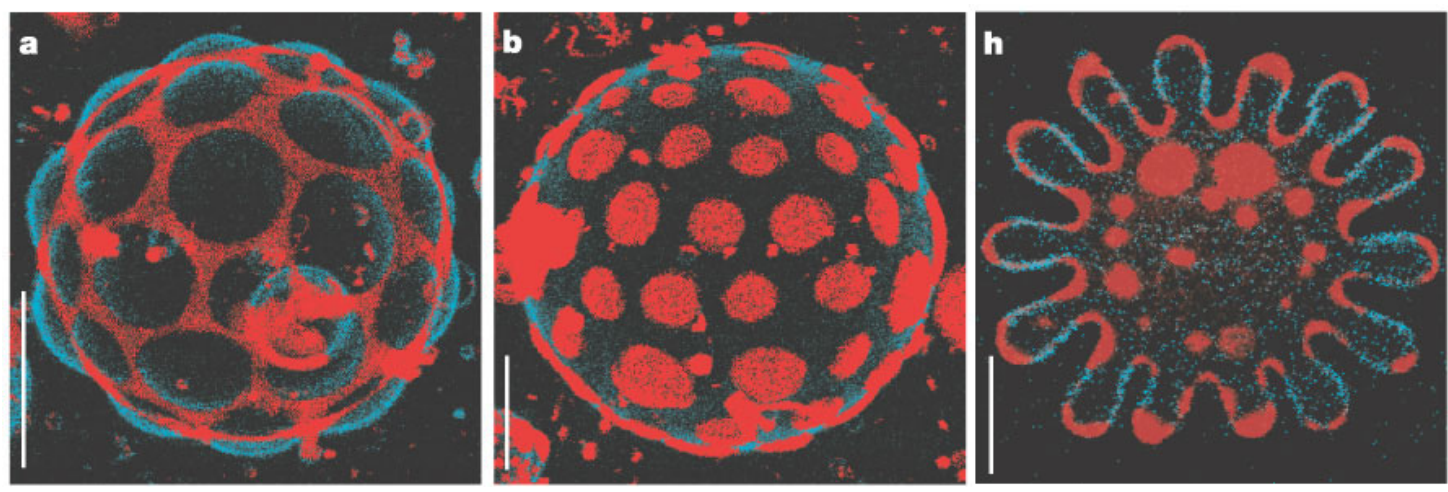

Figure 1.5: Two-photon microscopy images of GUVs where $L_{\beta}$ and $L_{\alpha}$ phases are shown in blue and red, respectively. The two leftmost images show the arrangement of the $L_{\beta}$ phase into hexagonal and inverted hexagonal structures. The rightmost image is the transversal projection of a osmotically deflated vesicle, exhibiting the preference of the $L_{\beta}$ to accommodate into low-curvature regions. The scale bar is $5 \mu \mathrm{m}$. Image reproduced from Baumgart et al. [32].

polar head-group and a rigid ringed structure, that fits into the voids between sphingolipids, hence functioning as a spacer) together with fairly cylindrical lipids, such as dioleoylphosphatidylcholine (DOPC). These studies have shown the phase separation of $\mathrm{L}_{\alpha}$ and $\mathrm{L}_{\beta}$ phases into domains characterized by length scales in the order of few micrometers (see images $a$ and $b$ in Fig 1.5 . $8-1114$ However, domains observed on these model systems are orders of magnitude larger than the nanometer-scale lipid rafts occurring in real cells. This length scale discrepancy, has been attributed to the absence of membrane proteins and the underlying cytoskeleton, entities which have been hypothesized to impose additional constraints in the limited size of lipid rafts.

Another interesting feature observed in this model system is the confinement of phase-separated domains within regions of the membrane with well defined curvature. For example, experiments with osmotically deflated vesicles collapsing into branched structures, like the one shown in the rightmost panel of Fig. 1.5 , have exhibited the preference for $\mathrm{L}_{\alpha}$ and $\mathrm{L}_{\beta}$ domains to accommodate into low and high curvature regions, respectively. ${ }^{32} \mathrm{~A}$ similar behavior has been observed in micropipette aspiration experiments, where tight bilayer tubes are pulled out from large, phase separated vesicles. The absence of the disordered phase within the highly-curved tubes confirms the preference of the stiffer $\mathrm{L}_{\beta}$ domains for flatter regions. ${ }^{33}$ In addition, segregation of phase-separated domains has also been observed in planar bilayers supported on patterned surfaces with high and low curvature regions. ${ }^{34}$

So far, we have presented some examples where externally imposed geo- 

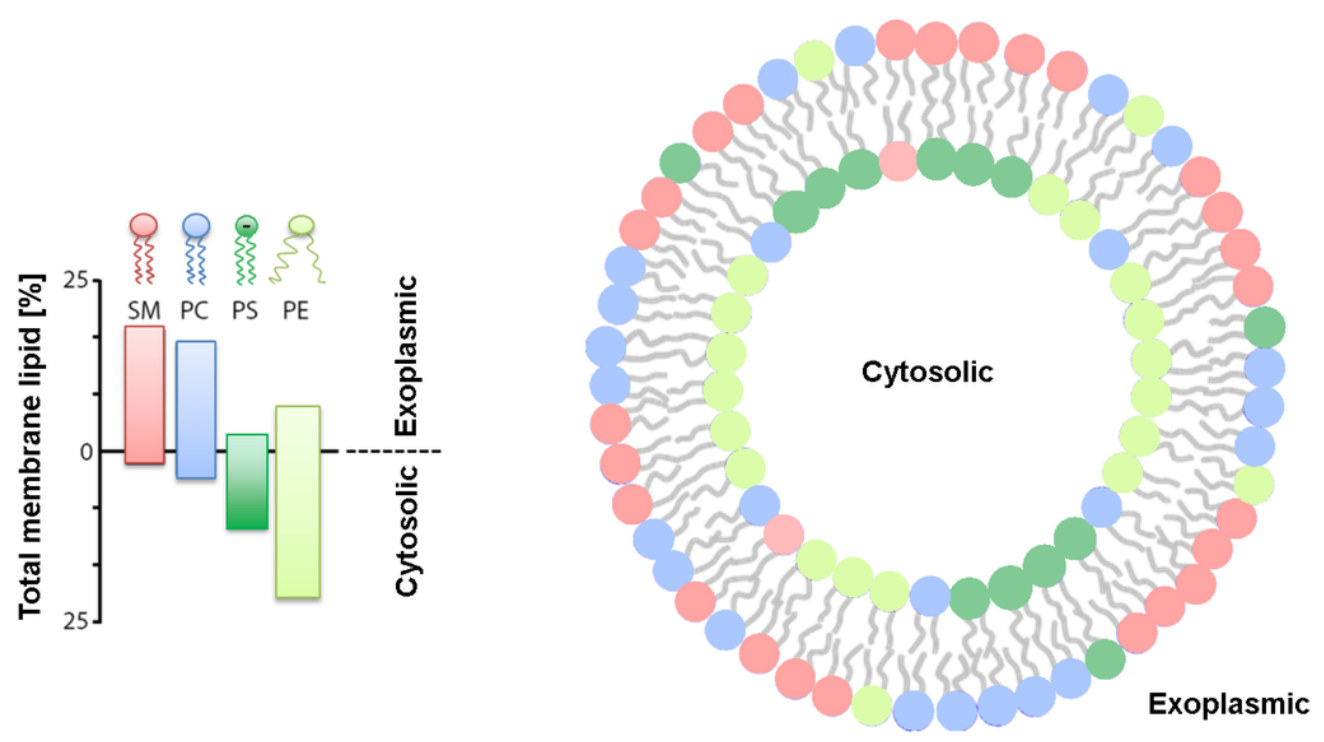

Figure 1.6: Schematic of the composition asymmetry between the inner and outer monolayers of mammalian's plasma membrane. Image reproduced from Marquardt et al. [35].

metric constraints modulate the lateral organization of $\mathrm{L}_{\alpha}-\mathrm{L}_{\beta}$ phase-separated, model membranes. A common feature in all these examples is that the composition of the two apposing bilayer leaflets are symmetric. However, it is well known that the inner and outer monolayers of biological membranes, have a rather asymmetric lipid composition. For instance, the plasma membrane of mammalian cells has an inner leaflet rich in phosphatidylserine (PS) and phosphatidylethanolamine (PE). These lipid families usually display an invertedcone geometry with the head group at the vertex. In contrast, the outer leaflet is usually enriched by sphingolipids and phosphatidylcholine (PC), which results in lipids with conical geometry (n schematic representation of the mammalian's plasma membrane is shown in Fig 1.6 . ${ }^{35}$ Since the monolayer spontaneous curvature is (to a large extent) an additive quantity of the intrinsic curvature of its individual lipids, an asymmetric lipid composition across the mammalian plasma membrane, will result in monolayers with opposite (complementary) spontaneous curvatures.

The complementary spontaneous curvatures between the apposing monolayers in an asymmetric membrane will help to relieve the stress induced by the isochoric deformation of the lipid tails, needed to prevent the formation of voids in the hydrophobic interior. In an optimal situation (and also neglecting the curvature shift due to the bilayer thickness), these curvatures will have the same magnitude and opposite signs (let's say $C_{\mathfrak{u}}=C$ and $C_{l}=-C$ for 


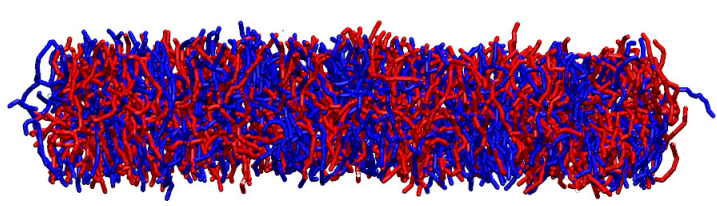

(a) Mixed bilayer

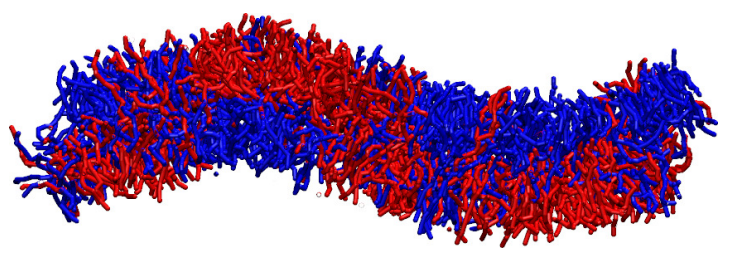

(b) Sorted bilayer

Figure 1.7: Shape-modulated bilayer due to the lateral sorting of lipids. Coneshaped lipids are shown in red and inverted-cone ones are shown in blue.

the upper and lower monolayers, respectively), therefore resulting in a tensionless bilayer with a finite spontaneous curvature, $\mathrm{C}$. This particular occurrence of monolayer spontaneous curvatures resulting in a curved, tensionless bilayer will be referred to as "commensurate".

If the monolayers spontaneous curvatures are not commensurate, there will be a residual stress to be distributed between the two leaflets. This stress redistribution will ultimately induce deviations on $\mathrm{C}_{\mathfrak{u}}$ and $\mathrm{C}_{l}$ from their tensionless values. This situation is most likely to occur in closed bilayers, such as vesicles or membranes tubes, where the composition of the two leaflets is highly asymmetric, that is, if the outer monolayer is almost entirely populated by coneshaped lipids whereas the inner monolayer is occupied by inverted-cone ones. This scenario corresponds to the simplest and perhaps most intuitive case of saturation between the two leaflets of the membrane. In the third chapter, however, we will see that, in a more general sense, the saturation state of the two monolayers will be dictated by the overall composition of the system.

Far from saturation, the stress can be further relieved via a coordinated insertion of the "wrong" lipid species into otherwise homogeneous domains in the two monolayers (for example insertion of cone-shaped lipids into a domain of inverted-cone ones in the lower monolayer coinciding with a complementary scenario in the upper monolayer). The net result of this process will be a local modulation of the membrane shape, where the curvature of the two apposing monolayers are commensurate (as shown in Fig.1.7b). Thus, this interplay between the compositional asymmetry across the bilayer and the local curvature, provides a mechanism for the formation of lipid rafts. Furthermore, it accounts for the composition asymmetry between the inner and outer leaflets of many naturally occurring biological membranes. 


\subsection{Outline}

Several models have been proposed to describe the lateral sorting of lipids and its interplay with membranes curvature in model bilayer mixtures comprised of two or three lipid species together with cholesterol. Although most of these models explicitly distinguish the composition on each bilayer leaflet (subject to normalization constrains), only few of them consider their difference in curvature. This simplification is valid for planar bilayers in the absence of strong shape fluctuations and curved surfaces with radius of curvature much larger than the bilayer thickness. However, important contributions to the lateral sorting of lipids may arise when this assumption is not met, examples of these are membrane tubes, necks, stalks, pore rims, etc. This work introduces a simple phenomenological model that explicitly takes this curvature difference into account, resulting in further contributions to the free energy of mixing. Furthermore, this new model offers a straightforward way to measure the spontaneous curvature of the individual lipids species, a task that nowadays remains an experimental challenge. The model is validated with molecular dynamics simulations of binary-mixture, self-assembled structures with different geometries and under different segregation conditions.

This dissertation is arranged as follows: in the next chapter, the physical background and the computational details needed to carry out our simulations, are discussed. It starts by introducing the coarse-graining concept and the two qualitatively different approaches to achieve the dynamical description of a systems with a reduced number of degrees of freedom. There, the relevant interactions to be accounted for, when developing lipid bilayers models, are pointed out. The second section introduces the implicit-solvent, coarsegrained model by Hömberg et al., together with a generalization aimed to describe multicomponent systems. In the third section, the integration schemes needed to carry out the simulation in different thermodynamic ensembles (microcanonical, canonical, semi-grand canonical and tensionless), are described. The last section describes the domain-decomposition parallelization strategy implemented in this work.

The first section of Chapter 3 gives a short review of the phenomenological, mean-field models aiming to describe the free energy of the membrane. Following a fairly chronological order, it starts with the models by Canham and Helfrich, which pointed out the importance of the elastic properties and the curvature of the bilayer, to duly describe the geometrical conformations of closed vesicles as well as the power spectra of shape fluctuations of extended membranes. The section continues with the seminal models by Leibler and Andelman, accounting for the heterogeneity of the membrane via a coupling term between the local curvature and density (or composition) fields. Finally, 
the section closes with subsequent generalizations that incorporate the bilayer structure of the membrane into the density field.

The second section of Chapter 3 introduces a new model which, beside incorporating contributions from the free energy of mixing on each monolayer, also accounts for their difference in curvature. As will be seen, this generalization will account for packing effects that are particularly important in systems whose local curvature is comparable to the inverse bilayer thickness. Finally, in section 3 , the influence of a possible curvature dependence on the elastic properties of the system is investigated.

Chapter 4 concerns itself with the results of simulations of bilayer structures with different geometries and segregation conditions. The first section of this chapter deals with the setup of the coarse-grained model introduced in Chapter 2 and presents some preliminary measurements used to validate the chosen parametrization. The second section investigates the elastic and demixing properties of planar bilayers and, from these studies, many of the parameters of the mean-field model introduced in Chapter 3 are evaluated. The third section investigates the correlation between the local curvature and composition in planar bilayers, with thermally induced shape fluctuations. From this study, the spontaneous curvatures of the two lipid species in the system are evaluated. Finally, the chapter closes with an extension of the findings with planar bilayers to highly-curved membrane tubes, where the composition of the two monolayers are close to saturation conditions. The results obtained from these simulations show that the proposed phenomenological model duly accounts for the important packing effects emerging at high curvatures.

Chapter 5 introduces an alternative method to measure the spontaneous curvature of lipid monolayers. This method is based on the idea that the insertion of lipids with unknown curvature into an otherwise tensionless and unconstraint inverted structure (where the intrinsic lipids curvature coincides with the geometrical curvature of the inverted aggregate), will induce expansions or contractions of the inverted phase. These deformations are proportional to the concentration of the lipids with unknown curvature. The chapter starts with a brief introduction to self-assembled inverted structures and the idea of lipid curvature additivity in these inverted phase, pointing out important effects due to the stretching of lipids at the intersection between three or more inverted aggregates. In the second section, a method to prevent these stretching effects is provided and the thermodynamics of the suggested systems is investigated, resulting in a new method to determine the intrinsic curvature of the species from pressure measurements. The chapter closes with the simulation results in these systems and the comparison with the results obtained in Chapter 4.

The thesis closes with a summary of the most important findings and an 
outlook for possible extensions. 


\section{Chapter 2}

\section{Model}

This chapter introduces the physical background and computational details required to carry out the simulations. In the first section, we present an overview of the coarse-graining techniques, making a clear distinction between the systematic and minimalistic approaches. Within the minimalistic description, we provide a brief guide of the relevant features to be taken into account when developing models for lipid bilayers. In the second section, we present a detailed description a generalization to the implicit-solvent, coarse-grained model by Hömberg and Müller ${ }^{36}$. This generalization is required to study the structural and dynamical properties of bilayers that contain more than one lipid species. The third section concerns itself with the algorithms needed for the computational implementation of the model in different thermodynamic ensembles. Finally the chapter closes with the description of the parallelization strategy adopted to speedup the simulations.

\subsection{Coarse-Graining}

Even when quantum effects can be completely neglected, the classical study of mesoscopic systems can be carried out at different levels of description. At the most fundamental level, every single atom, in each molecule of the system, is included. This description comes with a finely tunned parametrization of the interaction force fields between particles. Depending on the chemical complexity of the molecules, these interactions may contain terms accounting for specific chemical bonds, such as bond-bending, dihedral and torsion angle potentials, as well as non-bonded interactions, including van der Waals forces and other electrostatic interactions. ${ }^{2137+42}$ Since the aim of this description level is to reproduce local properties of the system in great detail (for instance, typically atomistic simulations of lipid bilayers include few hundred lipids spanning an area 
$\sim 1-10 \mathrm{~nm}^{2}$ and over time scales on the order of few nanoseconds ${ }^{43}$, 45 , the correct parametrization of the force field is crucial. Usually, these phenomenological parameters are tuned to fit the results of quantum calculations of small fragments of the molecules. $\frac{46-48}{48}$ Another characteristic feature of the atomistic description is that, since it includes the positions and momenta of all particles in the system, its dynamics is completely deterministic (for closed systems), i.e., it is solely dictated by Hamilton equations of motion.

Evidently, the large number of particles needed to study mesoscopic systems, such as small lipid vesicles or planar bilayers exhibiting the formation of composition domains (typically consisting of $10^{4}-10^{6}$ lipids and characterized by time scales in the order of $10^{-9}-10^{-6}$ seconds), makes unfeasible the implementation of such a detailed description.

On the opposite side of this spectrum of coarse-grained models is the thermodynamical level. Here, instead of particle positions and momenta, the relevant variables are the dynamical invariants of the system, such as mass, momentum and energy. In contrast to the atomistic description, there are no equations of motion describing the evolution of the system: i.e., the relevant variables settle in the equilibrium state. Consequently, the characteristic time scale becomes infinite.

In between these two levels, lies the so-called coarse-grained description, where the system is represented by a smaller number of degrees of freedom than the ones it actually has. Of course, such a representation is not unique. In general, different representations will be characterized by different length and time scales. $\frac{42 / 49-53}{53}$ Within the systematic or bottom-up approach to coarsegraining, the relevant properties from a more detailed level of description are used to parametrize a new model at a coarse-grained scale. For instance, in the united atom model for polymers, each carbon atom is grouped with its bounded hydrogen atoms into a single interaction center. The sole fact of reducing the number of particles in the system, makes the new representation more computationally efficient than its atomistic counterpart, while still preserving the main architectural features of the original molecule. Furthermore, by coarse-graining the carbon-hydrogen bonds the characteristic time scales associated with them (the fastest fluctuating modes in the atomistic description) have been also eliminated. This is an additional contribution to the computational efficiency of the coarse-grained model, which allows to use longer integration steps during simulations. However, this immediately leads to a question on the dynamics of the new system: how does the Hamiltonian description has to be modified after coarse-graining? Instead of addressing this question directly, pioneers of the systematic description (Kirkwood ${ }^{54}$, Green ${ }^{[55}$, Zwanzig ${ }^{[56157}$ and Mori ${ }^{[58}$ among others) searched for criteria that guarantee the existence and thermodynamic 
stability of coarse-grained models.

The basic idea behind the systematic approach consists in evaluating the multidimensional potential of mean force, effective Hamiltonian or free energy surface, $\mathrm{U}_{\mathrm{CG}}$, of the reduced degrees of freedom, $\underline{49}\{\mathbf{R}\}$,

$$
\exp \left(-\frac{\mathrm{U}_{\mathrm{CG}}[\{\mathbf{R}\}]}{\mathrm{k}_{\mathrm{B}} \mathrm{T}}\right)=\int \mathcal{D}[\{\mathbf{r}\}] \exp \left(-\frac{\mathcal{H}_{\mathrm{AA}}[\{\mathbf{r}\}]}{\mathrm{k}_{\mathrm{B}} \mathrm{T}}\right) \delta(\mathbf{R}-\mathbf{R}[\{\mathbf{r}\}]),
$$

where $\mathcal{H}_{\mathrm{AA}}$ is the Hamiltonian in the detailed, all-atom description, characterized by the set of coordinates $\{\mathbf{r}\}$. This definition for the effective Hamiltonian results in an identical probability distributions for both the coarse-grained and the detailed systems. Therefore, the coarse-grained description should ultimately yield the correct thermodynamics. There is, however, a major drawback that usually prevents a direct use of this exact result: there is no guarantee that $\mathrm{U}_{\mathrm{CG}}$ can adopt any particular functional form. In general, it will consist of a complicated collection of multi-body interactions. 59

In order to obtain a tractable and computationally efficient description, several methods have been developed to approximate the expressions for $\mathrm{U}_{\mathrm{CG}}$ in terms of pairwise interactions. The idea behind these methods is to reproduce specific properties from reference simulations with the detailed, all-atom model. For example, in the Boltzmann inversion ${ }^{60}-62$ approach (which relies on the uniqueness theorem by Henderson ${ }^{63}$ ), $\mathrm{U}_{\mathrm{CG}}$ is iteratively approximated by matching the structure of the original system, via the radial distribution function $g_{\text {AA }}(\{\mathbf{r}\})$. This approach is suited to parametrize the potential of mean force in diluted or semi-diluted systems, where high-order density correlations are unimportant. A drawback of this methods is that the resulting $\mathrm{U}_{\mathrm{CG}}$ is particularly sensitive to properties of the reference system, such as temperature, pressure and composition and, therefore, it is hardly transferable to study other systems. An alternative approach that partially avoids this inconvenience is the force matching ${ }^{6465}$ method. The aim of this method is to match the average force on the coarse-grained interaction centers to the one expected from the all-atom system. The matching procedure starts with a given functional for the effective Hamiltonian. It usually consists of a long-rang Coulomb contribution, parametrized by the charge of the pseudo atoms, whereas the short-range interactions are accounted for by cubic splines, parametrized by knot values, which are realizations of the interaction potential at predetermined, discrete distances. Then, the effective Hamiltonian is obtained by finding the optimal values of the pseudo-charges and knot values, which minimize the sum of squared deviations between the atomistic forces and those obtained from the coarse-grained description. By explicitly including different geometries and different temperatures, the transferability issue is overcome. This systematic approach to coarsegraining has been extensively used over the last three decades to explore a 
plethora of phenomena in different scientific areas and, in particular, it has a long-standing tradition within the polymer physics community. $[3766-68$

A completely different philosophy for the development of coarse-grained models is the so-called minimalistic or top-down approach. In contrast to the systematic scheme, this paradigm does not attempt to derive a new level of description from an already well established and finely described one. Instead, it directly proposes an Ansatz for the minimal set of relevant interactions and length scales, needed for the emergence of the desired mesoscopic phenomena. An immediate consequence of this approach is the loose identification of the chemical components, which can not be tracked back to an specific atomistic description. Nevertheless, the relevant interactions may be designed to reproduce the essential properties of a whole group of chemical compounds. An

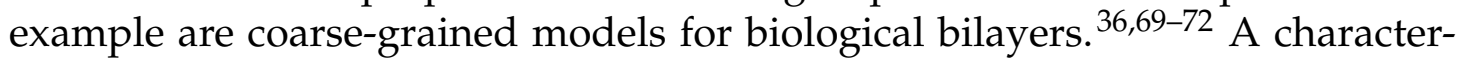
istic property of these bilayers is the amphiphilic nature of their constituting lipids, that usually have a hydrophobic tail, comprising two long fatty acid chains and an hydrophilic, polar head-group. When in solution, tails line up against one another in order to avoid the unfavorable contact with water, leaving the head-groups exposed at both sides of the bilayer. The key ingredient in this description seems to be the effective repulsion between the hydrophilic and hydrophobic groups, instead of the underlaying, specific mechanisms originating this repulsion. Another essential feature for this amphiphilic nature is the connectivity of molecules within a single chain, which prevents the macroscopic phase separation of the hydrophilic and hydrophobic blocks. $\frac{15}{18}$

Another aspect related to the amphiphilic architecture (but not to the chemical specificity) which has a large influence on the morphological aggregation of lipidic systems, is the volumetric ratio between the hydrophobic and hydrophilic blocks. ${ }^{73-77}$ The experimental evidence shows that lipids whose tail and head-groups have a similar size, tend to aggregate into bilayer structures (such as flat membranes or cylindrical tethers) whereas lipids with small headgroups and large tails, aggregate into inverted phases, where head-groups accommodate on the surface of cylindrical or spherical geometries, with tails pointing in the outward direction. Conversely, lipids with short tails and large heads also aggregate with heads over spherical or cylindrical geometries, but with tails pointing inwards. These two remarks emphasize the importance of preserving the amphiphilic structure when accounting for the lipid architecture in the coarse-grained description.

Within the aggregation mechanism described above, the interaction of the hydrophobic and hydrophilic blocks with solvent particles, seems to play a major role. Indeed, the proper characterization of the head-solvent interactions is crucial when hydrogen bonds have to be included. However, in a more coarse- 
grained description, it may be advantageous to completely remove the solvent and effectively incorporate its effects within the interactions among heads and tails. $16136169778-81$ This approach will result in an enormous speedup of simulations, since the number of solvent molecules is a quantity that scales with the system size. However, the development of such models requires a high degree of physical intuition and their validity can only be inferred after comparing their predictions with experiments. Fortunately, during the last decade, the development of implicit-solvent models is rapidly increasing and nowadays there is a general picture of the relevant interactions needed to reproduce the correct physics in lipid bilayers. In the following section we will introduce an implicitsolvent, coarse-grained model, which has been proved to reproduce the correct thermodynamic behavior and phase diagram of lipid bilayers. ${ }^{36}$ Furthermore, we will generalize this model to study the correlation between the geometrical conformations and the amphiphilic characteristics of mixed bilayers.

\subsection{Coarse-grained, solvent free model}

In this work, we extend the coarse-grained, solvent free model developed by Hömberg et al. ${ }^{36182}$ The aim of this generalization is to study static and dynamic properties of bilayer structures composed by a mixture of lipid species differing by the type and size of their polar head-groups. The model incorporates two contributions: i) bonded interactions, acting solely between closely connected monomers within a single chain (i.e., statistically independent chains) and ii) non-bonded interactions, potentially acting on any pair of beads and having a large influence on the packing properties of the system. This section presents a detailed description of these interactions.

\subsubsection{Bonded interactions}

The bonded Hamiltonian consist of two terms. The first term considers lipids as a linear (single-tailed) collection of $\mathrm{N}$ beads, held together by identical harmonic springs with force constant $k_{s}$ and rest length $l_{0}$ (as shown in Fig.2.1),

$$
\frac{\mathcal{H}_{\mathrm{b}}^{\mathrm{spr}}}{\mathrm{k}_{\mathrm{B}} \mathrm{T}}=\frac{\mathrm{k}_{\mathrm{s}}}{2} \sum_{i=1}^{\mathrm{N}-1}\left(\mathrm{r}_{\mathrm{i}, \mathrm{i}+1}-\mathrm{l}_{0}\right)^{2},
$$

where $r_{i, i+1}=\left\|\mathbf{r}_{i+1}-\mathbf{r}_{i}\right\|, \mathbf{r}_{i}$ is the position vector of the $i$-th bead in the chain, $k_{B}$ is the Boltzmann constant and $T$ is the temperature of the system.

Analogously to the freely-joined model, this bead-spring description results in a Gaussian probability distribution function for the chain mean-square end-to- 
end distance, ${ }^{8384} R_{\mathrm{eo}}^{2}=\left\langle\left(\mathbf{r}_{\mathrm{N}}-\mathbf{r}_{1}\right)^{2}\right\rangle$. However, it is computationally more efficient, since it avoids the expensive evaluations associated with the constrained bond length in the freely-joined model.

Due to their relatively low molecular weight (i.e., degree of polymerization), biologically relevant, membrane-forming lipids are characterized by high orientation correlations along the tail. ${ }^{49}$ This makes it necessary to extend the beadspring description with an energy contribution that favors the orientation along the chain (i.e., enhances chain stiffness). Intuitively, the simplest way to include this is by means of the bond angle, even though there are more elaborated models including torsion angles and even cross-coupling terms between the bond stretching, bond angle and torsional components. $\frac{3840}{1 n}$ this model, incorporation of orientation correlations follows the simplest approach. However, instead of the bond angle, its cosine is computed, since this computational implementation is more efficient.

The aforementioned contributions result in the following expression for the bonded Hamiltonian of the model

$$
\frac{\mathcal{H}_{\mathrm{b}}}{\mathrm{k}_{\mathrm{B}} \mathrm{T}}=\frac{\mathrm{k}_{\mathrm{s}}}{2} \sum_{i=1}^{\mathrm{N}-1}\left(\mathrm{r}_{i, \mathrm{i}+1}-\mathrm{l}_{0}\right)^{2}+\mathrm{k}_{\mathrm{b}} \sum_{i=2}^{\mathrm{N}-1}\left(1-\cos \theta_{\mathrm{i}}\right),
$$

where $\theta_{i}$ is the angle between bonds at the $i$-th bead, i.e., $\cos \theta_{i}=\hat{\mathbf{r}}_{i-1, i} \cdot \hat{\mathbf{r}}_{i, i+1}$, with $\hat{\mathbf{r}}=\mathbf{r} / \mathrm{r}$, and $k_{\mathrm{b}}$ is the strength of the angular interaction.

\subsubsection{Non-bonded interactions}

The bonded interactions described in the previous section are intended to impose a gross constraint on the accessible configurations to closely connected beads. However, they do not affect far neighbors (which can come to close contact for sufficiently large chains), nor the monomers in different chains. Here, these contributions are accounted for by a phenomenological Hamiltonian, expressed in terms of local monomer densities, $\rho$. This Hamiltonian resembles a third-order virial expansion for the excess free energy, though it is assumed to be valid for all densities, not only in the limit where they become vanishingly small. ${ }^{[5]}$ This way, the non-bonded Hamiltonian reads:

$$
\frac{\mathcal{H}_{\mathrm{nb}}^{\prime}\left[\left\{\hat{\rho}_{\xi}\right\}\right]}{k_{\mathrm{B}} T}=\int \frac{\mathrm{d}^{3} \mathrm{r}}{\mathrm{R}_{\mathrm{eo}}^{3}} \hat{\rho}_{\alpha}(\mathbf{r})\left[\frac{\nu_{\alpha \beta}}{2} \hat{\rho}_{\beta}(\mathbf{r})+\frac{w_{\alpha \beta \gamma}}{3} \hat{\rho}_{\beta}(\mathbf{r}) \hat{\rho}_{\gamma}(\mathbf{r})\right],
$$

where the Einstein sum convention over repeated Greek sub-indexes (i.e. bead species) has been used. In order to keep simple notation, the following derivation will only consider a mixture of two lipid species. Although, it is straightforward to relax this constraint. Each lipid has two components: i) a hydrophobic 

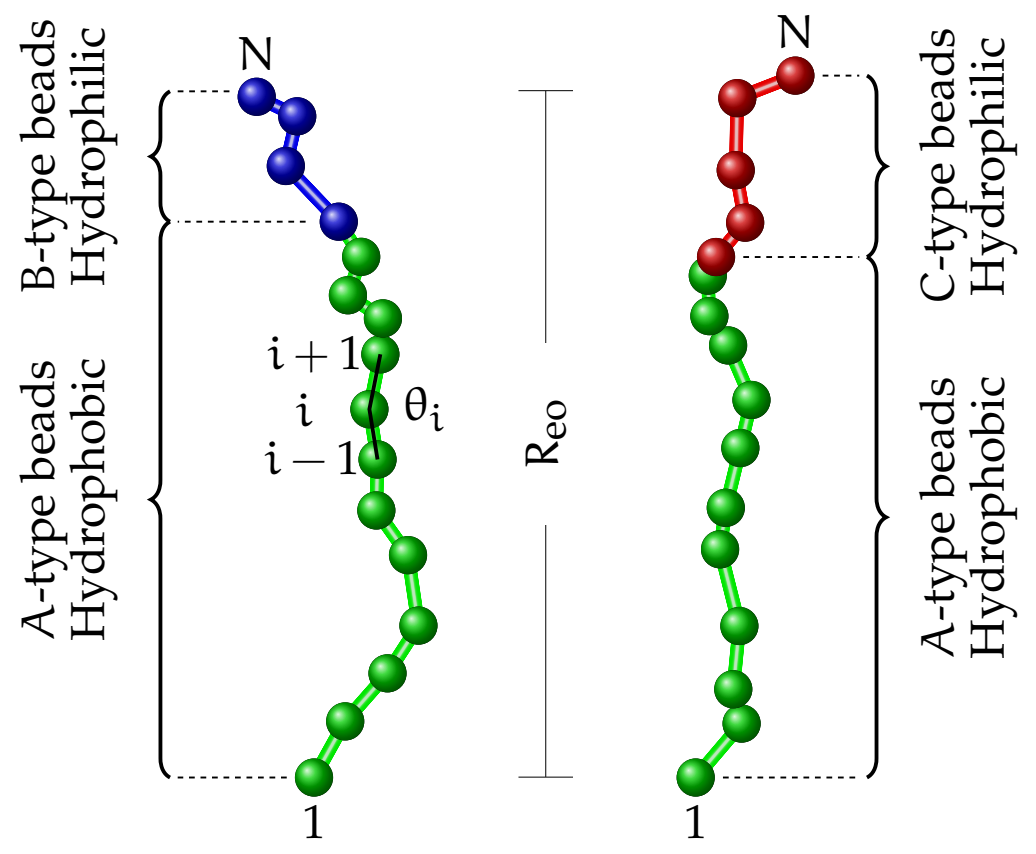

Figure 2.1: Chain architecture of the lipids considered in this study.

tail and ii) a hydrophilic head-group. The hydrophobic beads, labeled as $A$, will be common to all lipids. On the contrary, the hydrophilic beads will be different for each species, and labeled as B and C. Both architectures comprise the same total number of beads, $N$, though the relative length of head-group, $N_{\alpha}$, and tail, $\mathrm{N}_{\mathrm{A}_{\alpha}}=\mathrm{N}-\mathrm{N}_{\alpha}$, blocks, may differ among the species, as depicted in Fig.2.1. The densities appearing in this expression are re-defined as:

$$
\hat{\rho}_{\alpha}=\frac{R_{\mathrm{eo}}^{3}}{N} \sum_{i=1}^{n N} \delta\left(\mathbf{r}_{i}-\mathbf{r}\right) \delta_{\alpha t_{i}},
$$

where $n$ is the total number of chains in the system and $t_{i}$ stands for the species of the $i$-th bead. These functions have been normalized to account for the average number of $\alpha$-type beads within a unit volume $R_{e o}^{3}$. Furthermore, with this re-definition of densities, the non-bonded Hamiltonian becomes an explicit function of the particles coordinates, a necessary requirement for particle-based simulations. Nevertheless, the sharp delta function in this expression makes infeasible the computational implementation of systems described by such a Hamiltonian. Later on, these densities will be further re-defined in order to obtain a computationally tractable description of the system. 


\section{Expansion coefficients}

Now is time to address the expansion coefficients in the phenomenological Hamiltonian, Eq 2.4. As will be seen, these coefficients have a major influence on the thermodynamical properties of the system. We begin this discussion appealing to the hydrophobic nature of the lipid tails: when in solution, tails will melt into a dense liquid, in order to avoid the unfavorable contact with water. In a solvent free model, however, this scenario might be better described as a thermodynamic state where the hydrophobic melt coexists with its infinitely diluted vapor, i.e., at a zero coexistence pressure. This way, we can use the single component version of Eq.2.4 to find an approximate expression for the equation of state of the hydrophobic interior, yielding:

$$
\mathrm{P} \approx \frac{\mathrm{k}_{\mathrm{B}} \mathrm{T}}{\mathrm{R}_{\mathrm{eo}}^{3}}\left[\frac{v_{\mathrm{AA}}}{2} \rho_{\mathrm{A}}^{2}+\frac{2 w_{\mathrm{AAA}}}{3} \rho_{A}^{3}\right]
$$

A second expression relating these coefficients is obtained from the dimensionless, inverse compressibility or bulk modulus,

$$
\kappa N \equiv\left(R_{\text {eo }}^{3} \rho / k_{B} T\right)(\partial P / \partial \rho)_{T}=v_{A A} \rho+2 w_{A A A} \rho^{2} .
$$

Evaluating these expressions at the coexistence density, $\rho_{\text {coex }}=-3 v_{A A} / 4 w_{A A A}$, we find a way to relate the expansion coefficients $v_{A A}$ and $w_{A A A}$, with macroscopic, equilibrium properties of the lipid species:

$$
v_{\mathrm{AA}}=-2 \frac{\mathrm{\kappa N}+3}{\rho_{\mathrm{coex}}} \quad \text { and } \quad w_{\mathrm{AAA}}=\frac{3}{2}\left(\frac{\kappa \mathrm{N}+2}{\rho_{\text {coex }}^{2}}\right) .
$$

An important aspect to notice is the opposite sign of these coefficients. This is an immediate consequence of the coexistence requirement and justifies the choice of a third-order expansion for the non-bonded Hamiltonian. $85[86$ At low densities (i.e., large intermolecular distances) a slight increase of density will result in a pressure drop $\left(v_{\mathrm{AA}}<0\right.$, as shown in Fig 2.2). This reflects the large-distance dominance of attractive interactions, which further promote the aggregation of the melted phase. On the other hand, at high densities, the excluded volume interactions come into play and prevent the system from collapsing. A typical example of this, is the harsh repulsion of Lennard-Jones potentials at short intermolecular distances. Macroscopically, this is manifest by a positive slope of the $P$ vs $\rho$ curve (i.e., $w_{\text {AAA }}>0$ ), in the high density regime.

In contrast to tails, head-group beads should be highly soluble in water. It is therefore reasonable to assume they will be in good-solvent conditions, where the close contact with solvent molecules is favored over other headgroup beads. $\frac{2021}{2}$ In this case, interactions with solvent are zero and the goodsolvent assumption immediately sets the sign of both expansion coefficients: 
they have to be non-negative. This also implies that the qualitative behavior of the equation of state will be left unchanged if we set one of them to zero. In this work, we chose $w_{\mathrm{BBB}}=w_{\mathrm{CCC}}=0$, in agreement with the high-solubility picture (in which low density interactions play the main role), that provides the lowest pressure mismatch when the hydrophilic densities approach the coexistence density of the hydrophobic interior (see Fig 2.2). In order to further reduce this mismatch, it is necessary that the second order coefficients become small when compared to $w_{\text {AAA }}$.

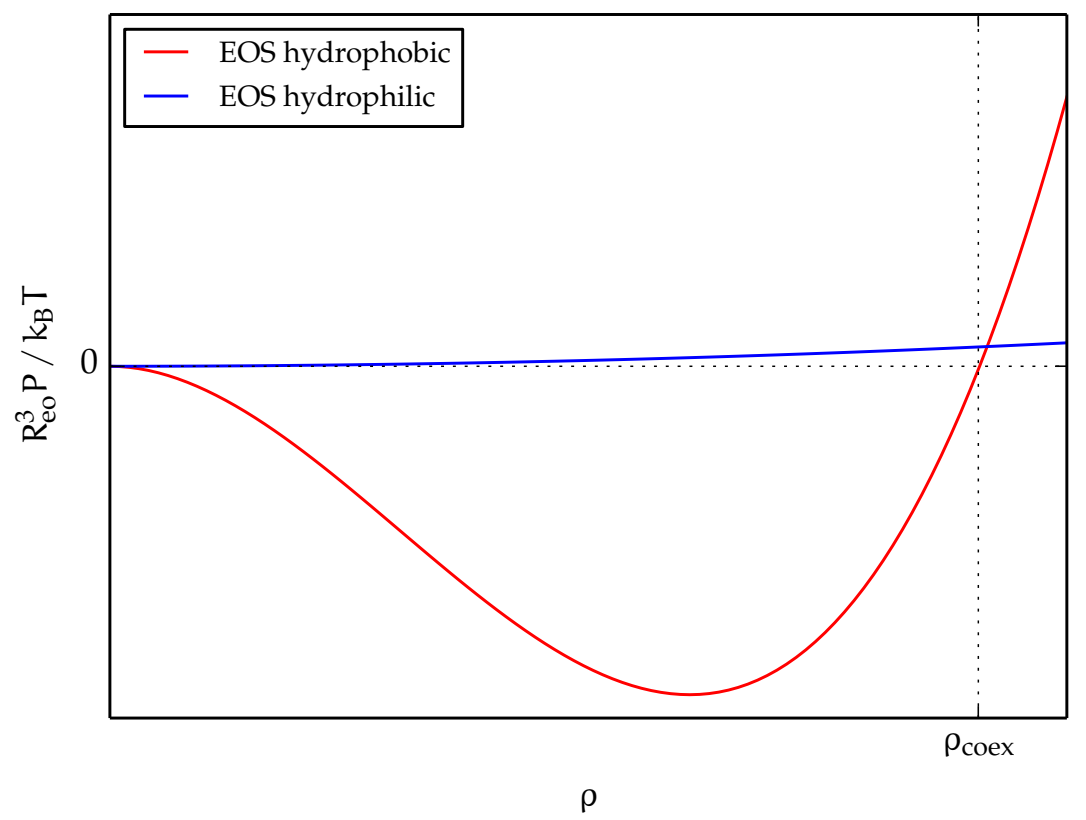

Figure 2.2: Equation of state of pure hydrophobic and hydrophilic beads at coexistence.

The interaction between beads of different species, $v_{\alpha \beta}$, is associated with the incompatibility or Flory-Huggins parameter, ${ }^{8788} \chi_{\alpha \beta} \mathrm{N}$, for each pair of different bead species $(\alpha, \beta) \cdot{ }^{85189}$ For the hydrophobic-hydrophilic interactions we have:

$$
\chi_{A B} N \equiv \rho_{\text {coex }}\left[v_{A B}-\frac{1}{2}\left(v_{A A}+v_{B B}\right)\right] .
$$

This parameter quantifies how different are the $\alpha-\beta$ interactions from those between pure substances, $\alpha-\alpha$ and $\beta$ - $\beta$. If $\chi_{\alpha \beta} N<0$, the species will mix well, whereas, if $\chi_{\alpha \beta} N>0$, it will be energetically more favorable to phaseseparate, which should be the case for the hydrophobic-hydrophilic interactions. We choose our parameters so that $\chi_{A B} N=\chi_{A C} N$ and, from now on, we 
denote them by $\chi_{\mathrm{th}} \mathrm{N} \geqslant 0$ (where the new subindex stands for tail-head). Concerning the hydrophilic-hydrophilic interactions, we will explore both the high and low incompatibility regimes and, to keep a consistent notation we replace $\chi_{\mathrm{BC}} \mathrm{N} \rightarrow \chi_{\mathrm{hh}} \mathrm{N}$.

For simplicity and since they do not change the qualitative behavior of the system, the rest of third-order coefficients, involving at least one hydrophobic species, are set equal to $w_{A A A}$, whereas those involving pure hydrophilic beads are set to zero. Then, the final mapping of the non-bonded expansion coefficients to macroscopic observables of lipid bilayers will be as follows:

$$
\begin{aligned}
v_{\mathrm{AA}} & =-2 \frac{\mathrm{kN}+3}{\rho_{\mathrm{coex}}}, \\
v_{\mathrm{AB}} & =v_{\mathrm{AC}}=\frac{\chi_{\mathrm{th}} \mathrm{N}}{\rho_{\mathrm{coex}}}+\frac{v_{\mathrm{AA}}+v_{\mathrm{BB}}}{2}, \\
v_{\mathrm{BC}} & =\frac{\chi_{\mathrm{hh}} \mathrm{N}}{\rho_{\mathrm{coex}}}+\frac{v_{\mathrm{BB}}+v_{\mathrm{CC}}}{2}, \\
v_{\mathrm{BB}} & =v_{\mathrm{CC}}=0.1, \\
w_{\mathrm{AAA}} & =w_{\mathrm{AAB}}=w_{\mathrm{ABB}}=\frac{3}{2}\left(\frac{\mathrm{kN}}{\rho_{\mathrm{coex}}^{2}}\right), \\
w_{\mathrm{ABC}} & =w_{\mathrm{AAC}}=w_{\mathrm{ACC}}=\frac{3}{2}\left(\frac{\mathrm{kN}}{\rho_{\mathrm{coex}}^{2}}\right), \\
w_{\mathrm{BBB}} & =w_{\mathrm{BBC}}=w_{\mathrm{BCC}}=w_{\mathrm{CCC}}=0 .
\end{aligned}
$$

\section{Weighted densities}

In the previous section, we discussed the role played by the expansion coefficients to set up, in broad terms, a meaningful thermodynamic behavior of the system. However, there is a key aspect of the model which still has to be modified in order to properly reproduce the important packing effects, observed in biological membranes. This aspect is the definition of densities in Eq2.5. As it stands, this definition is advantageous because of its explicit dependence on particles coordinates, which is a basic ingredient in molecular simulations. However, the impulsive forces originating from this definition of densities are hard to deal with, due to their short integration time requirements. More importantly, it completely overlooks the ability of real particles to allow certain degree of overlap when in crowded environments. In order to provide this feature to our model, the original molecular densities are convoluted with weighting functions, $w(r)$, that smooth and spread them over a characteristic region, $r_{c}$, which can be thought of as the radius of the associated coarse-grained segment. ${ }^{90191}$ Important requirements to be fulfilled by these weighting functions 
are: normalization, $\int \mathrm{d}^{3} w(\mathrm{r})=1$ (the total number of particles before and after reweighting must remain the same), vanish to zero at the cut-off distance $r_{c}$ and be differentiable everywhere (to avoid the emergence of impulsive forces).

In the previous discussion on the equation of state of the hydrophobic bead species, we identified third-order terms with the typical harsh repulsions at short intermolecular distances, whereas second-order terms account for attractive interactions at large scales. This suggest the use of different weighting functions for the second- and third-order densities. In this work we follow the same approach as Hömberg and Müller ${ }^{\sqrt{36182}}$ and set the second-order weighting function as follows:

$$
\vartheta_{2}(r)=A \begin{cases}\left(r_{c}-a\right)^{3}, & 0 \leqslant r<a \\ 2 r^{3}-3\left(a+r_{c}\right) r^{2}+6 a r_{c} r-3 a r_{c}^{2}+r_{c}^{3}, & a \leqslant r<r_{c} \\ 0, & r_{c} \leqslant r\end{cases}
$$

where $A$ is a normalization constant. Notice that the interpolation between the constant and zero potential regions is done via a cubic spline, which guarantees a smooth match at the end points, $a$ and $r_{c}$. The third-order weighting function

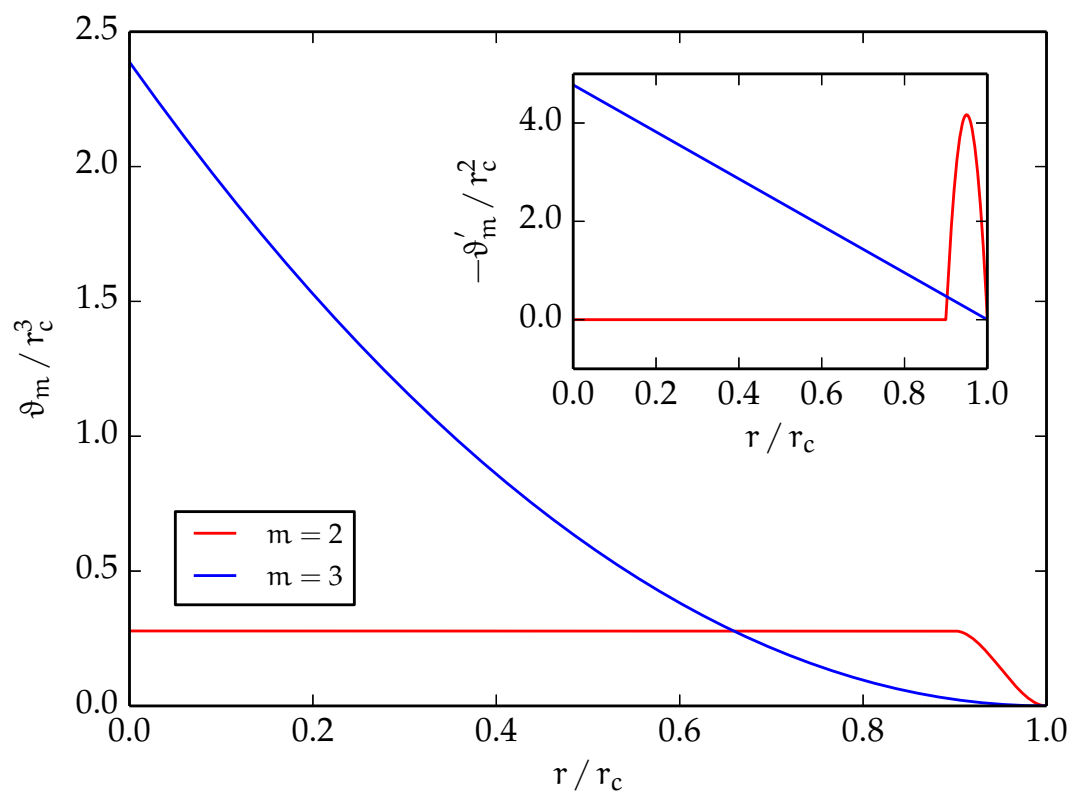

Figure 2.3: Main plot: weighting functions for the second- and third-order densities (denoted by the subindex $m=2,3$ ), for $a=0.9 r_{c}$. Inset: Negative derivatives of these functions, which are proportional to the force between particles. 
is defined as:

$$
\vartheta_{3}(r)=\frac{15}{2 \pi} \begin{cases}\left(r_{c}-r\right)^{2}, & r<r_{c}, \\ 0, & r_{c} \leqslant r .\end{cases}
$$

These functions, together with their negative derivatives (which are proportional to the force between particles) are shown in Fig 2.3. Notice that a has been chosen so that second-order interactions act only in the long-range limit, confining particles within the cutoff radius $r_{c}$.

Using these functions, we define the new weighted densities of the model,

$$
\tilde{\rho}_{\mathfrak{m} \alpha}(\mathbf{r})=\frac{R_{\mathrm{eo}}^{3}}{N} \sum_{i=1}^{n N} \vartheta_{\mathfrak{m}}\left(\left|\mathbf{r}_{i}-\mathbf{r}\right|\right) \delta_{\alpha t_{i}} .
$$

With these new densities, we can rewrite the non-bonded Hamiltonian of the system, $\mathrm{Eq}, 2.4$, in a computationally convenient way

$$
\frac{\mathcal{H}_{\mathrm{nb}}}{\mathrm{k}_{\mathrm{B}} \mathrm{T}}=\sum_{i} \delta_{\alpha \mathrm{t}(\mathrm{i})}\left[\frac{\nu_{\alpha \beta}}{2 \mathrm{~N}} \tilde{\rho}_{2 \beta}\left(\mathbf{r}_{i}\right)+\frac{w_{\alpha \beta \gamma}}{3 \mathrm{~N}} \tilde{\rho}_{3 \beta}\left(\mathbf{r}_{i}\right) \tilde{\rho}_{3 \gamma}\left(\mathbf{r}_{i}\right)\right] .
$$




\subsection{Numerical implementation}

Using the coarse-grained, solvent free model introduced in the previous section, we perform molecular dynamics (MD) simulations of lipid bilayer structures under different thermodynamical conditions and geometries. These constrains require the use of several statistical ensembles. In this section, we provide a description of the numerical implementation of these ensembles.

\subsubsection{Model discretization}

The force field of this model is obtained by direct differentiation of the bonded and non-bonded contributions to the Hamiltonian, Eq 2.3 and Eq2.14, with respect to the beads coordinates

$$
\begin{aligned}
& \mathbf{F}_{i}^{\mathrm{spr}}=-\mathrm{k}_{\mathrm{s}}\left[1-\frac{\mathrm{l}_{0}}{\mathrm{r}_{\mathrm{i}, \mathrm{i}+1}}\right] \mathbf{r}_{\mathrm{i}, \mathrm{i}+1}, \\
& \mathbf{F}_{i-1}^{\text {ang }}=-\frac{k_{b}}{r_{i-1, i}}\left[\hat{\mathbf{r}}_{i, i+1}-\hat{\mathbf{r}}_{i-1, i} \cos \theta_{i}\right] \text {, } \\
& \mathbf{F}_{i}^{\text {ang }}=\frac{k_{b}}{r_{i-1, i}}\left[\hat{\mathbf{r}}_{i, i+1}-\hat{\mathbf{r}}_{i-1, i} \cos \theta_{i}\right]-\frac{k_{b}}{r_{i, i+1}}\left[\hat{\mathbf{r}}_{i-1, i}-\hat{\mathbf{r}}_{i, i+1} \cos \theta_{i}\right] \text {, } \\
& \mathbf{F}_{i+1}^{\text {ang }}=\frac{k_{b}}{r_{i, i+1}}\left[\hat{\mathbf{r}}_{i-1, i}-\hat{\mathbf{r}}_{i, i+1} \cos \theta_{i}\right] \text {, } \\
& \mathbf{F}_{i}^{\mathrm{nbd}}=-\frac{k_{\mathrm{B}} T R_{\mathrm{eo}}^{3}}{\mathrm{~N}^{2}} \sum_{j} \hat{\mathbf{r}}_{i j}\left\{v_{\mathrm{t}(\mathrm{i}) \mathrm{t}(\mathrm{j})} \vartheta_{2}^{\prime}\left(\mathrm{r}_{\mathrm{ij}}\right)\right. \\
& \left.+\frac{2 w_{\mathrm{t}(\mathrm{i}) t(j) \alpha} R_{\mathrm{eo}}^{3}}{3 \mathrm{~N}} \vartheta_{3}^{\prime}\left(\mathrm{r}_{\mathrm{ij}}\right)\left[\tilde{\rho}_{3 \alpha}\left(\mathbf{r}_{\mathrm{i}}\right)+\tilde{\rho}_{3 \alpha}\left(\mathbf{r}_{\mathbf{j}}\right)\right]\right\} .
\end{aligned}
$$

A remarkable feature of these equations is that both the bond angle and the non-bonded contributions decompose into a sum of two-body terms. Besides the coarse-grained nature and the absence of solvent, this feature adds to the computational efficiency of the model. It also simplifies considerably the evaluation of certain observables, such as the local pressure profile, whose moments provide information on the elastic properties of lipid bilayers.

The equations of motion Eq,2.15 will be integrated with the velocity Verlet algorithm. ${ }^{3892193}$ This is a second-order, two-step method which, beside its simplicity and easy implementation, is symplectic, time-reversible and conserves angular and linear omentum. The algorithm is as follows: in the first step (prior to the evaluation of forces), velocities are updated by half a time step $(\Delta t / 2)$ 
using old forces, then positions are updated by a full time step using the intermediate velocities

$$
\begin{aligned}
\mathbf{p}(\mathrm{t}+\Delta \mathrm{t} / 2) & =\mathbf{p}(\mathrm{t})+\frac{\Delta \mathrm{t}}{2} \mathbf{F}(\mathrm{t}), \\
\mathbf{r}(\mathrm{t}+\Delta \mathrm{t}) & =\mathbf{r}(\mathrm{t})+\Delta \mathrm{t} \mathbf{p}(\mathrm{t}+\Delta \mathrm{t} / 2) .
\end{aligned}
$$

In the second step, forces are evaluated and then velocities are updated by another half time step.

$$
\mathbf{p}(t+\Delta t)=\mathbf{p}(t+\Delta t / 2)+\frac{\Delta t}{2} \mathbf{F}(t+\Delta t) .
$$

A crucial factor for the implementation of any integration scheme is the adequate selection of the time step, $\Delta t .{ }^{94}$ Even though there is no standard procedure to do so, the ultimate goal is to avoid the long-timescale energy drift. For all simulations in this work, we have set $\Delta t=0.005 \tau$, for which we do not observe drifts larger than $1 \%$ in $10^{5}$ time steps (in the microcanonical ensemble), and where we have defined the unit time, $\tau=\sqrt{k_{B} T / r_{c}}$, which will be mapped to real time units once the self diffusion time of a single lipid is evaluated.

\subsubsection{Simulation ensembles}

\section{Canonical (NVT)}

Apart from the constant energy (microcanonical) simulations used to study the stability of the velocity-Verlet integrator and set the adequate time step, all simulations in this work are done at constant temperature. The prototypical statistical ensemble satisfying such requirement is the canonical ensemble, where the system is allowed to exchange energy with a thermal reservoir, but the constrains on constant volume and number of molecules are fixed. Allowing the system to sample the desired new set of canonical configurations implies a modification to the standard (Newtonian) molecular dynamics scheme described in the previous section. There is a large number of methods that have been developed to achieve this goal,, 95 ranging from simple re-scaling of velocities to the addition of stochastic terms governing the fluctuation and dissipation of energy in the system. In this work, we follow the methodology developed by Lowe (commonly referred as the Lowe-Andersen thermostat) ${ }^{9697}$ where, in every time step and after integrating the equations of motion using the velocityVerlet scheme, the relative velocities of all interacting pairs (in our case, those separated by a distance shorter than the cutoff radius for non-bonded interactions, $r_{c}$ ) are randomly reassigned with probability $\Gamma \Delta t$ (where $\Gamma$ is the collision frequency with the thermal reservoir). The velocity update is done such that 
the total linear and angular momenta of the pair is conserved, i.e., the velocity component along the line through their centers is taken from the distribution $\xi_{i j} \sqrt{2 k_{B} T / m}$, where $m$ is the bead mass and $\xi$ is drawn from a Gaussian distribution with zero mean and unit variance. This way, the resulting new velocities of the pair are given by

$$
\begin{aligned}
& \mathbf{v}_{i}=\mathbf{v}_{i}-\frac{1}{2}\left(\xi_{i j} \sqrt{\frac{2 k_{B} T}{m}}-\mathbf{v}_{i j} \cdot \hat{\mathbf{r}}_{i j}\right) \hat{\mathbf{r}}_{i j}, \\
& \mathbf{v}_{j}=\mathbf{v}_{j}+\frac{1}{2}\left(\xi_{i j} \sqrt{\frac{2 k_{B} T}{m}}-\mathbf{v}_{i j} \cdot \hat{\mathbf{r}}_{i j}\right) \hat{\mathbf{r}}_{i j} .
\end{aligned}
$$

Based on the original Andersen algorithm, the Lowe-Andersen thermostat satisfies detailed balance. This guarantees the proper sampling of the desired canonical distribution, regardless of the integration scheme (as long as it conserves energy). ${ }^{98}$ This is an advantage of the method when compared to other popular choices, such as the Nosé-Hoover thermostat ${ }^{99100}$ or dissipative particle dynamics (DPD), $101-104$ which require the use of more sophisticated integration mechanisms. An additional feature of this thermalization procedure is the contribution of the collision-induced impulsive forces to the fluctuating stress of the system, which translates into an increased viscosity and enhances the realization of liquid-like dynamics in the system. ${ }^{96 / 105}$

\section{Tensionless $\left(N P_{t} T\right)$}

The surface tension, $\gamma$, of a planar membrane parallel to the $x y$-plane is given by 106

$$
\gamma=\left\langle\mathrm{L}_{z}\left(\mathrm{P}_{\mathrm{n}}-\mathrm{P}_{\mathrm{t}}\right)\right\rangle, \quad \text { with } \quad \begin{aligned}
& \mathrm{P}_{\mathrm{n}}=\mathrm{P}_{z z}, \\
& \mathrm{P}_{\mathrm{t}}=\frac{1}{2}\left(\mathrm{P}_{x x}+\mathrm{P}_{y y}\right),
\end{aligned}
$$

where $L_{\alpha}$ is the lateral extension of the simulation box along the $\alpha$-axis (with $\alpha=x, y, z), P_{n}\left(P_{t}\right)$ the instantaneous value of the normal (lateral) pressure, and $P_{\alpha \alpha}$ are the diagonal components of the pressure tensor. In order to set the desired tension of the system, our particular implementation of the $N P_{t} T$ ensemble allows lateral fluctuations of the simulation box (due to the absence of solvent, i.e., $P_{n}=0$ outside the bilayer, fluctuations in the normal direction are irrelevant). Changing the area, $A(t)$, of the simulation region comes with a re-scaling of bead coordinates. This makes necessary the use of more involved integration schemes. Here, we follow the methodology developed by Kolb and Dünweg ${ }^{107}$ for the NPT ensemble and the later adaptation by Hömberg and Müller ${ }^{36182}$ for $\mathrm{NP}_{\mathrm{t}} \mathrm{T}$ ensemble with the present solvent free model. 
The algorithm is as follows: a new degree of freedom, $A(t)$, with mass, $Q$, and conjugated momenta, $\pi_{A}$, associated with the area of the simulation box, is introduced. Then, in the first integration step, all conjugate momenta and coordinates are updated by a half time step

$$
\begin{aligned}
p_{x / y}^{\prime} & =p_{x / y}(0)+\frac{\Delta t}{2} F_{x / y}(r(0)), \\
p_{z}(\Delta t / 2) & =p_{z}(0)+\frac{\Delta t}{2} F_{z}(r(0)), \\
\pi_{A}(\Delta t / 2) & =\pi_{A}+\frac{\Delta t}{2} L_{z} P_{t} \\
A(\Delta t / 2) & =A(0)+\frac{\Delta t}{2 Q_{z}^{2}} \pi_{A}(\Delta t / 2), \\
r_{x / y}^{\prime}(\Delta t) & =r_{x / y}(0)+\frac{A(0)}{A(\Delta t / 2)} \frac{\Delta t}{m} p_{x / y^{\prime}}^{\prime} \\
r_{z}(\Delta t) & =r_{z}(0)+\frac{\Delta t}{m} p_{z}(\Delta t / 2), \\
A(\Delta t) & =A(\Delta t / 2)+\frac{\Delta t}{2 Q^{2} L_{z}^{2}} \pi_{A}(\Delta t / 2) .
\end{aligned}
$$

After this step, particle coordinates are rescaled and new auxiliary momenta are set

$$
\begin{aligned}
r_{x / y}(\Delta t) & =\frac{\sqrt{A(\Delta t)}}{\sqrt{A_{0}}} r_{x / y^{\prime}}^{\prime} \\
p_{x / y}^{\prime \prime} & =\frac{\sqrt{A(\Delta t)}}{\sqrt{A_{0}}} p_{x / y}^{\prime} .
\end{aligned}
$$

In the second integration step, all conjugate momenta are updated by another half time step

$$
\begin{aligned}
\pi_{A}(\Delta t) & =\pi_{A}(\Delta t / 2)+\frac{\Delta t}{2} L_{z} P_{t} \\
p_{x / y}(\Delta t) & =p_{x / y}^{\prime \prime}+\frac{\Delta t}{2} F_{x / y}(r(\Delta t)) \\
p_{z}(\Delta t) & =p_{z}(\Delta t / 2)+\frac{\Delta t}{2} F_{z}(r(\Delta t)) .
\end{aligned}
$$

Finally, oscillations of the additional degree of freedom, $A(t)$, are damped via coupling to a Langevin piston 107

$$
\frac{\Delta t}{2} L_{z} P_{t} \rightarrow \frac{\Delta t}{2} L_{z} P_{t}-\gamma_{A} \frac{\Delta t}{2 Q_{L}^{2}} \pi_{A}+\sqrt{k_{B} T \gamma_{A} \Delta t / 2} \xi_{A},
$$

where $\gamma_{A}$ is the friction coefficient of the area "particle" and $\xi_{A}$ is random number drawn from a Gaussian distribution with zero mean and unit variance. 


\section{Semi-grand canonical $(N \Delta \mu \mathrm{T})$}

Most of our simulations consist of a blend of lipids which differ by the length and type of their polar head-groups. Studying the equilibrium properties of such mixed systems via MD simulations in the canonical ensemble can be a very inefficient process, especially when the system approaches the demixing critical point. ${ }^{108109}$ The reason for this is that the relaxation of composition fluctuations is entirely mediated by diffusion. Therefore, the characteristic time for the aggregation of composition domains within the bilayer, will be several orders of magnitude larger than the characteristic time of single chain conformations.

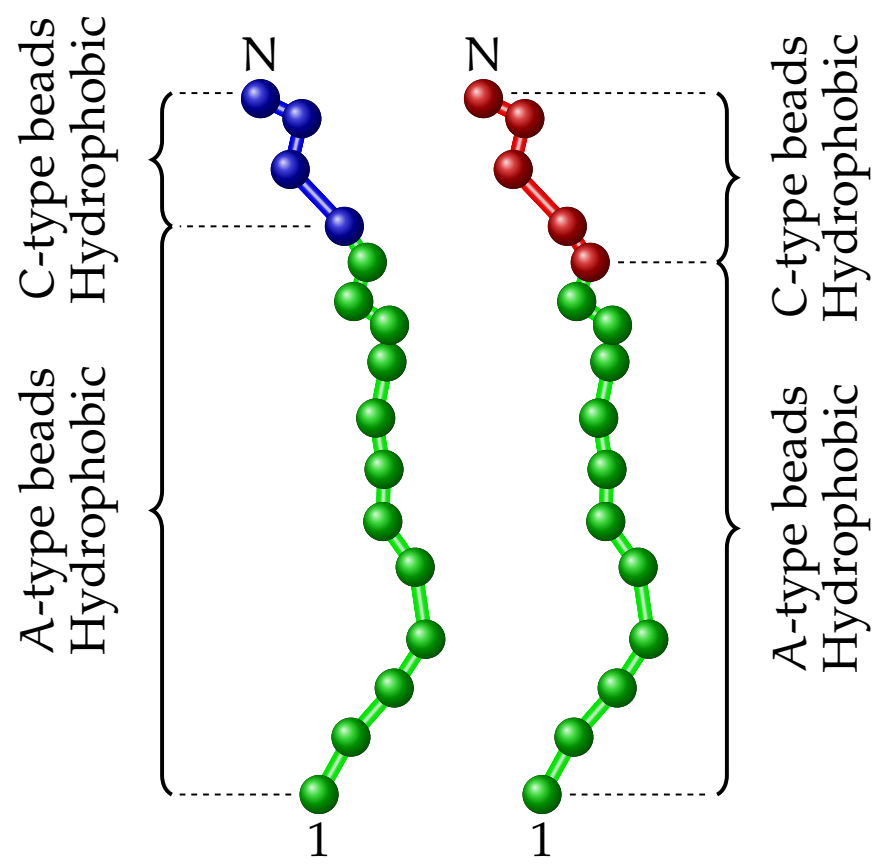

Figure 2.4: Mutation of lipid species in the semi-grand canonical implementation.

A common strategy to optimize the sampling of composition fluctuations is to work in the semi-grand canonical ensemble, where the total number of lipids in the system remains fixed, but the relative concentration of the species can fluctuate around an average value, set by their difference in chemical potential, $\Delta \mu .1101111$ Our numerical implementation of this ensemble is based on Monte Carlo moves with an acceptance probability

$$
P_{\mathrm{acc}}=\min \left\{1, e^{-(\Delta \mathrm{E} \pm \Delta \mu) / \mathrm{k}_{\mathrm{B}} T}\right\},
$$

where $\Delta \mathrm{E}$ is free energy difference of the system before and after the move. The algorithm is as follows: 
1. An arbitrary lipid is selected and the energy of the system is recorded.

2. The lipid is randomly mutated into a new species (see Fig.2.4) and the energy of this new configuration is evaluated.

3. The probability acceptance of the move is evaluated (using Eq 2.26) and compared to a random number, $\xi$ (drawn from an uniform distribution between 0 and 1 ).

4. The Monte Carlo move is accepted if $\xi<\mathrm{P}_{\text {acc }}$ or rejected otherwise.

\subsection{Parallelization}

A systematic study of the interplay between the lateral organization of lipids and the bilayer shape requires the simulation of systems characterized by different length scales. On the one hand, we simulate highly-curved systems, with lateral dimensions comparable to the bilayer thickness, $t_{h}$, (i.e., with a small number of lipids) and whose properties are highly influenced by local packing effects. On the other hand, we simulate planar bilayers, whose lateral dimensions are considerably larger than their thickness $\left(L_{x}, L_{y} \sim 10 t_{h}\right)$ and where shape fluctuations, as well as entropic effects, play a major role. The computational cost of these simulations is led by non-bonded interactions which, beside requiring the evaluation of local densities in every time step, scale as the square of the number of neighboring beads within a cutoff radius, $r_{c}$. Furthermore, some of the simulation are done in the vicinity of the demixing critical point, where the correlation of composition fluctuations is slowed down. These factors make necessary the use of parallelization algorithms to simulate large systems. This section is intended to provide a brief description of the parallelization strategy used in this work.

\section{Domain decomposition}

The parallelization strategy followed in this work is the domain decomposition.112 The simulation region is split into several domains and a single computational unit (denoted as processor from now on) is assigned to each of them. Each processor is in charge of calculating the forces and integrating the equations of motion of all particles within its boundaries. To evaluate the forces on particles at each domain boundaries, every process stores a copy of the positions and velocities of particles in the nearby domains that lay within its interaction radius (we will refer to this region as the shell of the process, as shown in Fig 2.5a). 


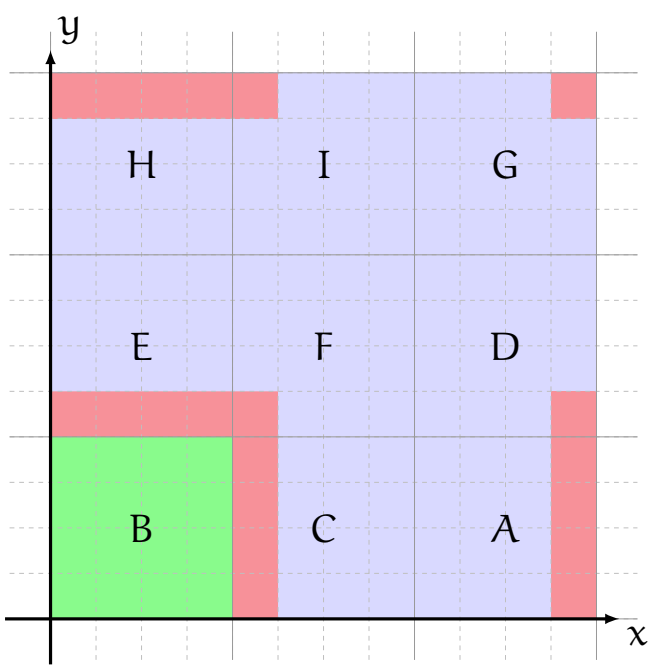

(a) Single domain (green) and its associated shell (red) which contains information from beads in its neighboring domains (those laying within its interaction radius). Other domains are delimited by the thin, continuous line.
East - West exchanges

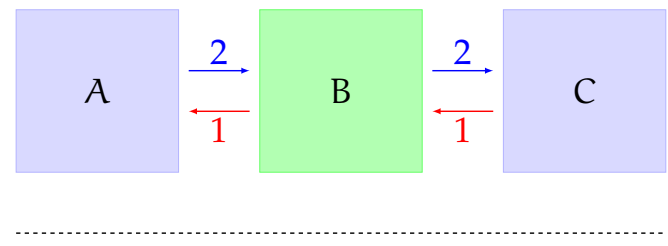

South - North exchanges

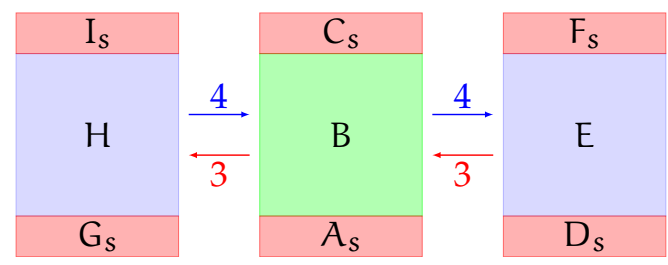

(b) Two-steps communication scheme between domains. Numbers indicate the sequence in which communication is performed and s-subscripted labels stands for shell information from the corresponding neighbor.

Figure 2.5: Domain decomposition of the simulation box.

The described approach is particularly suitable to study the dynamics of systems interacting via non-bonded interactions. However, difficulties arise when simulating chain-like structures that may include both pairwise or multi-body interactions, like the bonded interactions in our model. In such cases, a method is needed to deal with the connectivity between beads. Single processor algorithms can easily overcome such difficulty by making use of the linear memory storage of the beads in a single chain and evaluate all the interactions acting on these beads in a single loop. However, this ordering is completely lost in distributed computations after few simulation steps. We handle this difficulty by assigning a passport that has to be shared whenever information of a specific bead is required. This passport consist of a unique id number for the bead in the whole system (i.e., a bead index assigned at the beginning of the simulation) and the id number of the neighboring beads needed to evaluate its bonded interactions.

\section{Communication scheme}

This particular implementation takes advantage of the solvent-free nature of the simulation model and the geometry of planar bilayers (which are the most com- 
putationally demanding, due to their large number of lipids) and divides the simulation region into rectangular parallelepipeds, perpendicular to the bilayer plane. The process assigned to each division maintains two data structures: a core data structure, that stores the complete information (coordinates, velocities, weighted densities, passports, etc) of all beads within its boundaries, and shell data structure that stores the corresponding shell information. To facilitate the insertion and deletion of data, as particles are copied or moved between domains, this information is stored in linked lists. The communication exchange between processes has two steps (see Fig,2.5b):

1. Each processor exchanges information with its neighbors in the east and west directions. Processor B fills a sending buffer with the information of its own beads that lay within the interaction range of processor $A$. Once the message is sent, the communication buffer is ready to receive the incoming message from process $\mathrm{C}$. This information will be added to the shell data structure. Then the procedure is repeated, but with all processes exchanging information in the opposite direction, that is, sending to $C$ and receiving from $A$.

2. The same procedure as in 1 is repeated, but in the north and south direction. The only difference is that messages sent to adjacent processors now contain information from beads stored in the shell structure.

Therefore, this communication scheme only requires four data exchanges to collect the needed information from the eight surrounding neighbors of any domain.

\section{Neighbor list}

The main reason to adopt a parallelization strategy is to speedup the evaluation of the double sums in the expressions for the non-bonded interactions, Eq.2.15e. and the weighted densities, Eq2.13, whose naive implementation scales with the square of the total number of particles. The domain decomposition of the simulation region among $n_{p}$ processors would, ideally, reduce the original working load by a factor $\sim 1 / \mathrm{n}_{\mathrm{p}}^{2}$. However, the short-range nature of this nonbonded interactions may still result in an inefficient implementation if domains are larger than the cutoff radius of soft interactions, $r_{c}$, since there will be many non-interacting pairs within each domain. On the other hand, a drastic reduction of the domain size will create too much communication overhead, which is a major factor in detriment of efficiency for any parallelization scheme. To allow for low communication overhead and high efficiency in the detection of 
interacting pairs, each processor maintains a list with all the possible interacting neighbors, within a radius $r_{c}+r_{v}$, for every particle in its core data structure. ${ }^{38113}$ The Verlet radius, ${ }^{92} \mathrm{r}_{\mathrm{v}}$, serves as a buffer to regulate the evaluation of this neighbor list (which remains a time consuming task within each domain) and redistribution of particles between domains (once bead coordinates are updated). The main idea is that particles relocation and evaluation of the neighbor list will happen only after few simulation steps, when the maximum distance traveled by a single particle (in any domain) satisfies $\Delta r_{\max } \geqslant r_{v} / 2$.

\section{Ensembles}

Following this parallelization strategy, simulations in the microcanonical ensemble can be done with a single exchange of information per integration step. However, a more efficient implementation may result if, instead of evaluating the weighted densities in both the core and shell data structures, only the core evaluations are carried out and, afterwards, densities are broadcasted to the neighboring processes. In contrast to this free choice to achieve a better performance, the stochastic and pairwise nature of the thermalization algorithm makes mandatory the use of a new communication step, where each domain transfers its reassignment of particles velocities in the shell. To avoid multiple modifications, due to the repeated shell pairs in the different domains, each process evaluates a thermalization step only when the id of its core particle is larger than that of the particle in the shell.

The initial step for the semi-grand canonical parallelization is the random selection of possible chains undergoing a type mutation. This can be done by a single processor at the beginning of every integration step and broadcasted to all other domains within the first data exchange. Then, each mutation has to be carried on individually, in the domains spanned by the chain in turn. This type reassignment requires a new calculation of densities. Instead of evaluating these new densities using the original neighbor list (which stores all the interacting pairs, though in an unsorted way), a linked-cell list is constructed, exclusively, for the beads in the current chain.

Most of the simulations in this work were done at constant volume. A reason for this was to avoid the additional communication overhead, as well as the involved re-scaling of coordinates, required by the tensionless ensemble, which is not suitable for domain decomposition algorithms. Instead, $N P_{t} T$ simulations were implemented in a single processor and were mainly used for equilibration purposes, setting the desired average tension in the system. 
The final simulation algorithm used in this work is summarized in Fig.2.6.

$$
\begin{aligned}
& \text { First velocity-Verlet step } \\
& \text { If } \Delta r_{\max } \geqslant r_{\mathrm{v}} / 2 \text { : } \\
& \text { Update core } \\
& \text { Update neighbor list } \\
& \text { Update shell }
\end{aligned}
$$

Else:

Update shell

Evaluation of forces:

Evaluate bonded forces (passports)

Evaluate densities in the core

Update densities in the shell

Evaluate non-bonded forces

Second velocity-Verlet step

Thermalization

Update velocities in the core

Update velocities in the shell

Semi-grand canonical moves:

Construct linked-cell list

Swap chain type

Evaluate new densities and energy

Accept or reject the new type

Record observables

Figure 2.6: Domain decomposition algorithm for NVT and $N \Delta \mu T$ ensembles. 


\section{Benchmark}

The efficiency of this implementation was tested for a pre-assembled, planar bilayer with 42120 chains. Each chain comprised 16 beads, for a total of 673920 beads. We considered two lipid species, with a difference in chemical potential such that the average concentrations of both species were the same. In addition, the incompatibility between head-group beads was chosen to locate the system close to the demixing critical point. The results of this test are shown in Fig.2.7

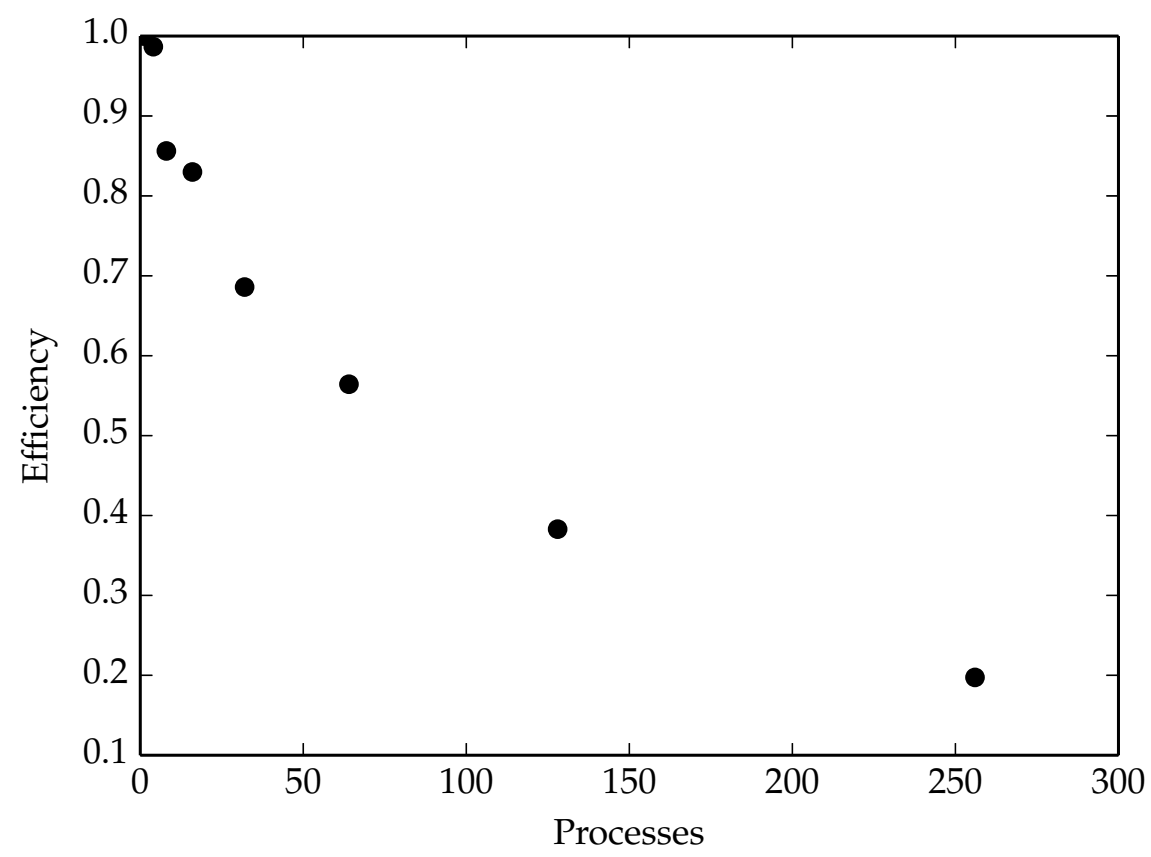

Figure 2.7: Efficiency of the domain decomposition implementation. 
CHAPTER 2. MODEL 


\section{Chapter 3}

\section{Phenomenological membrane models}

In this chapter we introduce the theoretical framework needed for the analysis of the coarse-grained simulations. The first section gives a broad overview of the mesoscopic, mean-field models for lipid membranes, starting from the first elastic description by Canham and Helfrich, to the extended models that account for the interplay between curvature and composition in those bilayers. Many of these models account for contributions to the membrane free energy from the energy of mixing of the individual monolayers. However, we point out that most of these models have only partially transfered the two-monolayer structure into the curvature field. Therefore, their applicability is restricted to the study of system whose lateral dimensions are much larger than the membrane thickness, i.e., they are not suitable for systems under strong curvature constraints. In the second section, we develop a new model that, beside the monolayers energy of mixing, explicitly considers the curvature of both leaflets and the intrinsic coupling between curvature and composition.

Among the predictions of this model is the existence of saturation curvatures, for which the modulation of lipids composition reaches its maximum. Furthermore, the model provides a quantitative description of the rate with which such saturation value is reached. This growth rate is what we define as the effective curvature-composition coupling. In the third section, we extend the model to account for high-curvature corrections that are due to the modification of the elastic properties of highly-bent membranes. 


\subsection{Early membrane models}

\subsubsection{Elastic models}

\section{Canham and Helfrich Hamiltonians}

Back in 1970, Peter Canham proposed one of the first models to duly account for the peculiar biconcave shape of human red blood cells, without considering

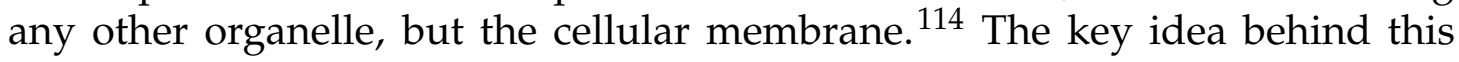
model was to identify bending (rather than stretching or torsion) as the relevant quantity that characterizes the free energy of the lipid bilayer. Relying on elastic theory, he proposed a generalization of the beam-bending energy to describe the elastic energy of the membrane:

$$
\mathcal{U}=\int_{\mathcal{S}} \mathrm{d}^{2} \mathbf{r} \frac{\kappa}{2}\left(\mathrm{c}_{1}^{2}+\mathrm{c}_{2}^{2}\right)
$$

where the integral extends over the whole surface of the bilayer, $\mathrm{k}$ is the bending modulus and $c_{1}$ and $c_{2}$ are the local, principal curvatures of the membrane. Few years later, in 1973, Wolfgang Helfrich found a generalization of this expression that became the cornerstone for most mesoscopic, mean-field models describing the thermodynamics of lipid bilayers. Instead of directly relying on elastic theory, Helfrich started from a general quadratic expansion for the membrane elastic energy in terms of the spatial derivatives of the local bilayer normal. Then, appealing to physical grounds and symmetry arguments, he justified the omission of irrelevant terms in the expansion. 115 The reasonings followed at that stage were similar to those used by Oseen ${ }^{116}$ and Frank ${ }^{117}$ when deriving the free energy of liquid crystals. Beside the curvature-independent term, $\gamma$, which accounts for energy contributions due to membrane stretching, the Helfrich generalization for the bilayer free energy is:

$$
\mathcal{U}=\int_{\mathcal{S}} \mathrm{d}^{2} \mathbf{r}\left[\frac{\kappa}{2}\left(\mathrm{c}_{1}+\mathrm{c}_{2}-\mathrm{C}_{0}\right)^{2}+\overline{\mathrm{k}} \mathrm{c}_{1} \mathrm{c}_{2}+\gamma\right] .
$$

Compared to the Canham's Hamiltonian, Eq.3.1, there are two additional coefficients in this expression. The first one is the spontaneous curvature of the bilayer, $\mathrm{C}_{0}$. Due to symmetry, this quantity should vanish in completely homogeneous membranes. However, the presence of heterogeneities may lead to a preferred curvature, thereby setting $\mathrm{C}_{0} \neq 0$. For example, this may be the case in membranes whose two apposing monolayers are composed of lipids with different chemical identities or packing geometries. The second coefficient, $\bar{k}$, is the so-called saddle-splay or Gaussian modulus. It accounts for the membrane 
preference to adopt concave/convex or saddle shapes. This contribution, however, may be omitted when studying membranes with closed geometry (such as closed vesicles) or idealizations where some of the lateral dimensions of the membrane extend infinitely (such as infinite planar membranes or cylinders). The reason for this, relies in the celebrated Gauss-Bonnet theorem, that relates the surface integral of the local Gaussian curvature, $\mathrm{K}=\mathrm{c}_{1} \mathrm{c}_{2}$, of a manifold $\mathcal{S}$, to the line integral of the geodesic curvature, $k_{g}$, along the contour of $\mathcal{S}$ and the Euler characteristic, $\chi(\mathcal{S})$ : a scalar invariant that describes the shape of a topological space regardless of how it's bent.

$$
\int_{\mathcal{S}} d^{2} \mathbf{r} K=2 \pi \chi(\mathcal{S})+\int_{\partial \mathcal{S}} \mathrm{d} s k_{g} .
$$

The immediate consequence of this result is that, for membranes without boundaries, the second term in the Helfrich Hamiltonian, Eq. 3.2. may be neglected, since it will integrate into a constant with no influence on the physical properties of the system. Taking this into account, the Helfrich Hamiltonian can be rewritten as:

$$
\mathcal{U}=\int_{\mathcal{S}} d^{2} \mathbf{r}\left[2 \kappa\left(H-C_{0}\right)^{2}+\gamma\right],
$$

were we have defined the mean curvature, $\mathrm{H}=\frac{\mathrm{c}_{1}+\mathrm{c}_{2}}{2}$. The practical use of this expression will still depend on the way curvatures are computed, which in turn will depend on the parametrization of the membrane.

\section{The Monge gauge}

One of the most appealing ways to describe membranes, thinking of them as extended objects, is the so-called Monge gauge. In this description, the local height, $h$, is measured with respect to a reference plane located at $z=0$, as shown in Fig. 3.1. This way, the coordinates of any point on the membrane are given by $\mathbf{R}=\mathbf{R}(x, y, h(x, y))$. Of course, this assumes that $h(x, y)$ is singlevalued and, thus it's restricted to the study of quasi-planar surfaces.

Within the Monge gauge, the mean curvature can be evaluated via the divergence of the membrane normal, $2 \mathrm{H}=-\nabla \cdot \hat{n}$. However, the resulting expressions allow for analytic ways to find equilibrium configurations (those that minimize the resulting Helfrich Hamiltonian) only in a very limited number of cases. For this reason, it is usually assumed that $h(x, y)$ is a smooth-enough function (i.e., $|\nabla h| \ll 1$ ), so that a small gradient expansion results in an appropriate description of membrane fluctuations. These simplifications yield the most common approximation to the Helfrich Hamiltonian:

$$
\mathcal{U}=\frac{1}{2} \int_{\mathcal{S}} \mathrm{d}^{2} \mathbf{r}\left[\kappa\left(\nabla^{2} \mathrm{~h}\right)^{2}+\gamma(\nabla h)^{2}\right] .
$$




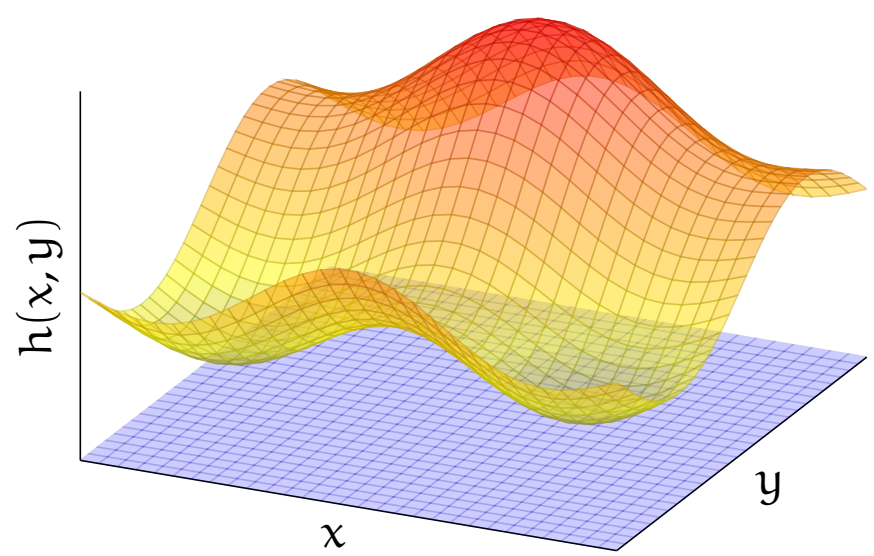

Figure 3.1: Monge parametrization of a planar membrane patch.

\section{Fluctuation spectra}

The quadratic dependence of the Helfrich Hamiltonian on curvature, Eq 3.5 , is enormously convenient when computing statistical averages and, in particular, it will provide an analytical path for evaluating equilibrium properties. Another key aspect of this harmonic model is that shape fluctuations are completely determined by two uncoupled contributions: out-of-plane deformations (bending) and in-plane compressions. By measuring the spatial correlation of these undulations, we can determine the conditions favoring the appearance of one kind of mode or another. To carry out this study, it is convenient to evaluate the Fourier expansion of height fluctuations:

$$
h(\mathbf{r})=\sum_{\mathbf{q}} h_{\mathbf{q}} e^{i \mathbf{q} \cdot \mathbf{r}} \quad \text { with } \quad \mathbf{q}=\frac{2 \pi}{L}\left(n_{x}, n_{y}\right), n_{x}, n_{y} \in \mathbb{N},
$$

where, for simplicity, we have assumed a membrane patch with equal lateral dimensions, L, replicating infinitely via periodic boundary conditions. With this definition the Helfrich Hamiltonian, Eq. 3.5, can be rewritten as follows:

$$
U_{\mathbf{q}}=\frac{L^{2}}{2} \sum_{\mathbf{q}}\left|h_{\mathbf{q}}\right|^{2}\left[\kappa q^{4}+\gamma q^{2}\right]
$$

Then, invocation of the equipartition theorem, which states that every quadratic term in the Hamiltonian contributes exactly $k_{B} T / 2$ to the average energy of the 
system, yields the desired expression for the membrane fluctuation spectra:

$$
\left\langle\left|h_{\mathbf{q}}\right|^{2}\right\rangle=\frac{k_{B} T}{L^{2}\left[k q^{4}+\gamma q^{2}\right]} .
$$

Now it is clear that compression modes will become dominant whenever $\mathrm{q}<\sqrt{\gamma / \mathrm{k}}$. Another remarkable feature of this expression is the simple dependence on the phenomenological parameters of the model. This is one of the reasons why measuring the fluctuation spectra has become one of the preferred techniques to determine the bending modulus of lipid bilayers.

\subsubsection{Beyond purely elastic models}

The Helfrich model has had many modifications since its introduction. An example of this is the protrusion field, introduced by Lipowsky in the early 90 's, 118 which accounts for the observed roughness of the membrane on lengthscales just above the bilayer thickness. This roughness is induced by the relative motion of individual lipids and its contribution to the fluctuation spectra has the same mathematical dependence on the wave vector than the compression modes. However, it is characterized by a different surface tension coefficient. $118-120$ There have also been generalizations accounting for the internal structure of the lipid tails inside the bilayer (described via a local orientational field, as it is usually done when studying liquid crystals) as well as the interactions among these additional degrees of freedom and membrane inclusions. ${ }^{1211122}$ This kind of models have been useful to elucidate essential conditions to regulate membrane-mediated interactions between inclusion as well as conditions for the stability of new membrane conformations, such as the rippled phase usually observed on highly-hydrated phospholipid bilayers. .123

\section{The Leibler model}

A common feature of all these models is that they consider membranes as homogeneous surfaces where the chemical identity of the constituting lipids does not play any role. However, the presumed existence of rafts, $\frac{3891124125}{12}$ the evidence of phase segregation in multicomponent membranes under the influence of high curvatures (such as those characteristic of membrane-pulling experiments) $32[33] 126-128$ and the well-known composition asymmetry between the apposing monolayers of certain types of cells and cellular organelles, $\frac{1291130}{12}$ are only some examples of the imminent role that inter- and intra-composition heterogeneities should play in the modulation of membrane shapes and the plethora of processes regulated by these membranes. 
It was Leibler who, in 1986, first proposed a model to account for these composition heterogeneities. Specifically, the model was aimed at incorporating the effect of freely-diffusing membrane inclusions, which were assumed to interact stronger with the polar head-groups than with the lipid tails. These asymmetric interactions result in an heterogeneous distribution of the adsorbed molecules, since they prefer to accommodate into regions where the bilayer is locally bent inwards/outwards, so that the contact with the polar head-groups is maximized/minimized. He proposed to incorporate these interaction into the continuous Helfrich description via a coupling term between membrane curvature and the local density field of inclusions, $\phi$ :

$$
\mathcal{U}_{\text {coup }}=\int_{\mathcal{S}} \mathrm{d}^{2} \mathbf{r} \lambda \mathrm{H} \phi,
$$

where the new phenomenological parameter, $\lambda$, quantified the strength and direction of the coupling interaction (in the following, this parameter will be denoted the intrinsic curvature-composition coupling). In addition, the model incorporated a power-series expansion in terms of density, accounting for the interaction between inclusions. $\frac{131}{13}$ Predictions of this model include the periodic curvature modulation of two-component vesicles, under strong segregation conditions, as well as the presence of shape instabilities, characterized by high-order bending modes. Beside the intrinsic coupling, such instabilities were triggered by the pressure difference between the interior and the exterior of the vesicle. This additional term in the free energy, was a later contribution by Andelman and coworkers, which directly coupled the pressure difference across the membrane with its total enclosed inner area. $132[133$

\section{Importance of the bilayer structure}

Subsequent generalizations focused on capturing the two-leaflet structure of lipid membranes. Although many of those new models fully transfered the dual character into the composition or density field, the corresponding mapping into curvature was only partially conferred, i.e., they assumed the curvature of apposing monolayers to have the same magnitude but opposite signs, accounting for the direction in which each monolayer locally bends (towards the hydrophobic interior or away from it). These generalizations exhibit the spontaneous formation of large vesicles, configurations that result unstable against lamellar or micellar phases in single-component systems. Such stabilization is possible due to the ability of vesicles with different compositions in the inner and outer leaflets to relieve the frustration of curvature energy. ${ }^{101134}$ Furthermore, phase separation in those systems may occur at finite length scales, i.e., their structure 
factor of composition fluctuations is characterized by the presence of high amplitude peaks at non-zero wave vectors. This provides an explanation for the formation of lipid rafts as a direct consequence of the curvature-composition coupling. ${ }^{141135}$ However, two caveats arise from these models. First, stability of the vesicular phase is only possible for compositions near the critical value for the lamellar-vesicular phase transition. Whether these conditions can be met in biologically relevant systems, has to be further investigated. Second, conclusions from these models are drawn under the assumption of isotropic curvatures, i.e., restricted to the study of only spherical vesicles.

In a complementary approach, Seifert showed the mechanism by which curvature-induced phase segregation in two-component vesicles favors the appearance of budding transitions on initially spherical vesicles. ${ }^{136}$ This was an important contribution to reinforce the hypothesis of the curvature-composition coupling as an essential mechanisms for the stabilization of curved bilayer morphologies and the concomitant asymmetric distribution of lipids across the membrane. However, recent experimental and theoretical findings on supported planar membranes, have pointed out the importance of distinguishing coupled and uncoupled phase separation between leaflets. These findings suggest that it is the tendency to phase-separate in one of the monolayers, together with a non-curvature dependent interaction (at least not explicitly) between leaflets, what induces a coupled segregation across the bilayer. ${ }^{[29137}$ This poses a question on the mechanisms regulating the correlated segregation of lipids in the two leaflets of the membrane: so far, the intrinsic curvature-composition coupling seems to be enough to explain this correlated segregation on curved morphologies. What is, however, its relevance to account for the same phenomena in planar membranes?

In the next section, we will introduce a new model able to describe the correlated segregation of lipids across the membrane, appealing to no other interactions but the elastic energy and the intrinsic curvature-composition coupling. The novelty of this description, when compared to the aforementioned ones, is that it will fully transfer the two leaflets architecture of the bilayer into both the local curvature and composition fields (i.e., it will relax the constraint of equal magnitude and opposite sign for the curvature of apposing monolayers). Beside the correlated segregation, this generalization will account for packing effects that become particularly important in systems characterized by curvatures comparable to the inverse bilayer thickness, such as those occurring in membrane-pulling experiments. 


\subsection{Composition-dependent free energy of mixing}

In the previous section we presented a brief overview of the mesoscopic, continuous models developed for the study of lipid membranes. We saw that, since its introduction more than 40 years ago, the first elastic models by Canham and Helfrich have been continuously extended to provide a more accurate description not only of membrane equilibrium shape deformations but also to account for more intricate phenomena, such as the partial phase segregation of their lipid constituents, as well as the underlaying mechanisms stabilizing these finite length-scale domains. A major breakthrough in this direction is the recognition that the intrinsic bilayer structure of the membrane provides a natural path to relieve the frustration of curvature energy: If we consider the membrane to be composed of lipids with different spontaneous curvatures (i.e., lipids with different volume ratios for their polar head-groups and tails), lipids with large head-groups will prefer to cluster into regions where the monolayer is bent towards the hydrophobic interior (regions where the accessible area per lipid is increased), whereas lipids with short head-groups will cluster in regions where the monolayer is bent in the opposite direction. This mechanism will confer an effective local curvature and lipid sorting, proportional to the difference in mol fractions between lipid species. The effectiveness or strength of such sorting is what we call intrinsic curvature-composition coupling. However, as we pointed out, although these models considered the composition of each leaflet independently, they also assumed the local curvature of apposing monolayers to have the same magnitude and opposite signs (since the local normals of the monolayers point in opposite directions). This description is suitable for the study of smooth surfaces in the absence of high curvatures. However, as local curvature becomes comparable to the inverse bilayer thickness, the curvature difference between apposing monolayers increases drastically. This would inevitably result in important packing effects, which can be accounted for by considering the individual curvatures of each leaflet. In this section we will introduce a new model that explicitly takes this curvature difference into account.

\section{Curvature and composition in the bilayer structure}

Consider a membrane consisting of two parallel leaflets separated by a distance $t_{h}$, which sets the bilayer thickness. A small patch of this model membrane is schematically represented in Fig.3.2, where subindices $u$ and 1 stand for the upper and lower monolayers, respectively. Each of these monolayers is characterized by its area, $A_{\mathrm{u} / 1}$, and radius of curvature, $\left|\mathrm{R}_{\mathrm{u} / 1}\right|=\left|1 / \mathrm{H}_{\mathrm{u} / 1}\right|$. In order to facilitate the analysis, we consider these two quantities to be expressed as functions of the bilayer midplane area, $A$, and curvature, $H$, as well as the bilayer 
thickness. In these terms, the corresponding quantities for each monolayer read:

$$
\begin{array}{cc}
\mathrm{H}_{\mathrm{u}}=\frac{\mathrm{H}}{1-\frac{\mathrm{th}_{\mathrm{h}}}{2} \mathrm{H}^{\prime}}, \quad \mathrm{H}_{\mathrm{l}}=\frac{-\mathrm{H}}{1+\frac{\mathrm{t}_{\mathrm{h}}}{2} \mathrm{H}^{\prime}} \\
\mathrm{A}_{\mathrm{u}}=\mathrm{A}\left(1-\frac{\mathrm{t}_{\mathrm{h}}}{2} \mathrm{H}\right), & \mathrm{A}_{\mathrm{l}}=\mathrm{A}\left(1+\frac{\mathrm{t}_{\mathrm{h}}}{2} \mathrm{H}\right) .
\end{array}
$$

Sign differences in these expressions stand for the fact that the local normals of apposing monolayers point in opposite directions. This means that convex curvatures on the upper leaflet, where the shielding hydrophilic region is bent towards the hydrophobic interior (i.e., increasing the monolayers area with respect to the bilayers midplane), will be paired to concave deformations of the lower leaflet, where the hydrophilic region is bent away from the bilayer midplane (i.e., resulting in a reduced area when compared to bilayers midplane). Furthermore, when writing these expressions, we have adopted the convention were convex deformations are assigned negative curvatures.

Beside its geometry, each leaflet patch is characterized by its lipid composition. For simplicity, our analysis will consider only two different lipid species. The total number of lipids on each patch will be denoted as $n_{\alpha}=n_{\alpha 1}+n_{\alpha 2}$, where the Greek index denotes the monolayer, i.e., $\alpha=\mathrm{u}, \mathrm{l}$, and numerical subindices stand for the different lipid species. The local composition or order parameter on each leaflet is defined by:

$$
-1 \leqslant \phi_{\alpha}=\frac{n_{\alpha 1}-n_{\alpha 2}}{n_{\alpha 1}+n_{\alpha 2}} \leqslant 1 .
$$

\section{Energy contributions}

The model incorporates three contributions to the free energy of the bilayer: the elastic energy, due to out-of-plane deformations of each monolayer, the free energy of mixing between lipid species and the intrinsic coupling between curvature and composition degrees of freedom. In this model, a possible inter-bilayer coupling has been omitted, because the interaction of hydrocarbon tails across the midplane is negligible.

The elastic contribution takes the form of a Helfrich Hamiltonian for each monolayer:

$$
\frac{F_{b e n}}{k_{B} T}=\frac{k_{m}}{2} A_{u}\left(H_{u}-C_{u}\right)^{2}+\frac{k_{m}}{2} A_{1}\left(H_{l}-C_{l}\right)^{2},
$$

where $C_{u}$ and $C_{1}$ are the spontaneous curvatures of the upper and lower leaflets, respectively. In this expression we have assumed that the bending rigidity, $\mathrm{k}_{\mathrm{m}}$, is the same for both monolayers. Furthermore, we have assumed that it is a 


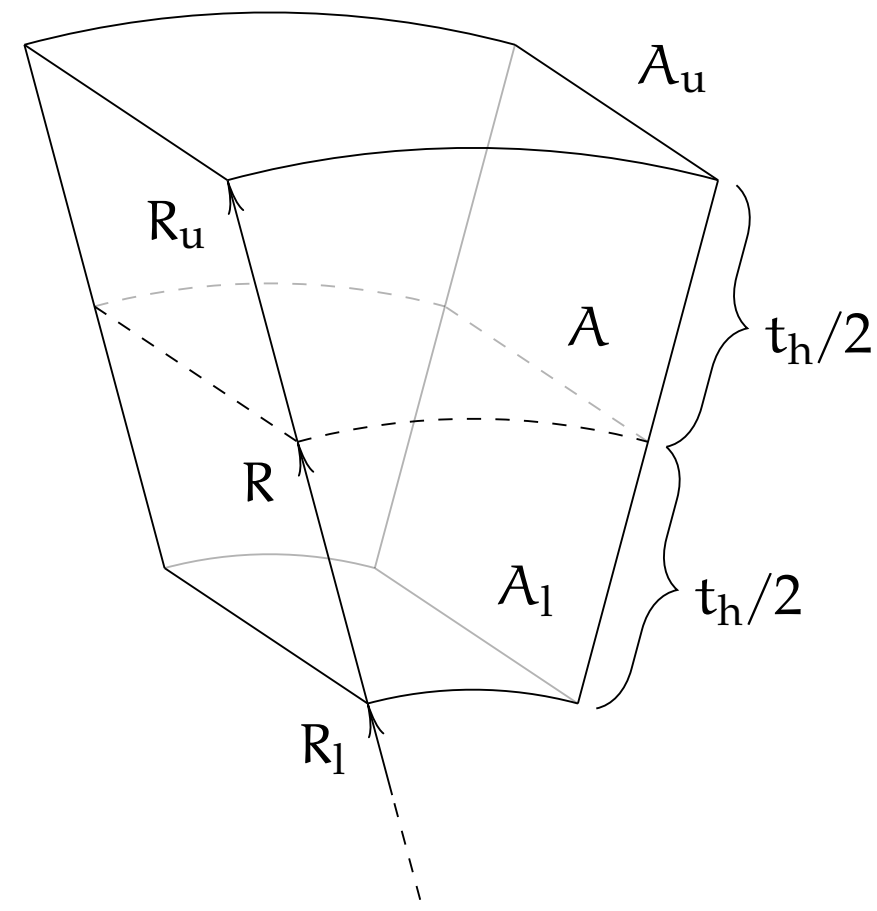

Figure 3.2: Schematic representation of a curved membrane patch, where the dotted surface represents the middle plane.

constant, independent of curvature and composition. The first of these assumptions is relaxed in the following section, where we consider an explicit quadratic dependence on the midplane curvature. Though the second assumption is not further justified from a theoretical point of view, a posteriori confrontation with the simulation results shows that, indeed, it provides an appropriate description of the bilayer bending resistance.

A natural way to account for the intrinsic curvature-composition coupling is via the spontaneous curvature of the individual monolayers. According to the geometrical picture previously described, these curvatures should be proportional to the composition difference between lipid species. In this work we will assume a simple linear dependence between these quantities:

$$
\mathrm{C}_{\mathrm{u} / \mathrm{l}}=\mathrm{C}_{0}+\left(\frac{\sigma_{\mathrm{m}}}{\kappa_{\mathrm{m}}} \lambda\right) \phi_{\mathrm{u} / \mathrm{l}}
$$

where $\lambda$ is the intrinsic curvature-composition coupling, $\sigma_{m}$ is the areal density of lipids per monolayer and $\mathrm{C}_{0}$ the average spontaneous curvature between the two lipid geometries. Later it will be shown that the peculiar form of the term in parenthesis recovers the standard definition of the intrinsic coupling (as 
originally introduced by Leibler in Eq.3.9p. Furthermore, it must be noticed that setting $\phi_{\mathrm{u} / 1} \pm 1$ in this definition, provides a one-to-one relation between the phenomenological coefficients in our model and the spontaneous curvatures of the lipid species (which will be denoted as $\mathrm{C}_{+1}$ and $\mathrm{C}_{-1}$ ):

$$
\lambda=\frac{C_{+1}-C_{-1}}{2 \eta_{m}} \quad \text { and } \quad C_{0}=\frac{C_{+1}+C_{-1}}{2},
$$

with $\eta_{\mathrm{m}}=\sigma_{\mathrm{m}} / \mathrm{K}_{\mathrm{m}}$. A gross estimation of the intrinsic coupling can be immediately drawn from this expression, since typical values for the areal density of lipids and the bending rigidity per monolayer are: $\sigma_{\mathrm{m}} \approx 2.0 \mathrm{~nm}^{-2}$ and $\mathrm{K}_{\mathrm{m}} \approx 10.0$ (in units of $\mathrm{k}_{\mathrm{B}} \mathrm{T}$ ). $\stackrel{3376}{\mathrm{It}}$ is also well-known that, irrespective of their sign, the spontaneous curvatures of biologically relevant lipids can be as large as $\left|C_{ \pm 1}\right| \approx 0.9 \mathrm{~nm}^{-1}$. 76 Therefore, we should expect to measure values of the intrinsic coupling bounded by $|\lambda| \lesssim 4.5 \mathrm{~nm}$ from our simulations.

Finally, the energy of mixing takes the form of a simple mixture for each monolayer: $: 8788$

$$
\frac{\mathrm{F}_{\text {mix }}}{\mathrm{k}_{\mathrm{B}} \mathrm{T}}=\sum_{\alpha} \mathrm{n}_{\alpha}\left[\frac{1+\phi_{\alpha}}{2} \ln \left(\frac{1+\phi_{\alpha}}{2}\right)+\frac{1-\phi_{\alpha}}{2} \ln \left(\frac{1-\phi_{\alpha}}{2}\right)+\frac{\chi}{4}\left(1-\phi_{\alpha}^{2}\right)\right],
$$

where $\alpha$ is the monolayer index, the first two terms within the square brackets stand for the entropy of the mixture and the last one is its enthalpy. The phenomenological coefficient accompanying this last term, $\chi$, is the so-called FloryHuggins parameter, which accounts for the non-ideality of the mixture, i.e., it quantifies the free energy of interaction between different lipid species. This parameter is not to be confused with those introduced in the parametrization of the coarse-grained model, Eq. 2.9 and Eq. 2.10. While these coarse-grained definitions accounted for the two-body interactions between beads of different species, either within a chain or among beads in different chains, the definition in this mean-field model, Eq.3.16, effectively accounts for all the interactions between chains or lipids, of different species.

\section{The joint composition description}

An important feature of this expression is that, beside compositions, it explicitly depends on the total number of lipids per leaflet, $n_{\alpha}$. For completely planar bilayers, the population of each leaflet should be on average the same. However, for highly-curved membranes, such as small vesicles or membranes tubes, the population should be proportional to the area of the corresponding leaflet and, therefore, to its curvature. To make this dependence explicit, we will set 
$n_{\alpha}=\sigma_{\mathrm{m}} A_{\alpha}$, where the weak quadratic dependence of $\sigma_{\mathrm{m}}$ on curvature is neglected. Furthermore, since it is the joint contribution of both leaflets what ultimately sets the thermodynamics of the system, we rather express the free energy of the membrane in terms of the average composition, $\psi$, and the composition difference between monolayers, $\phi$. These are defined by:

$$
-1 \leqslant \psi=\frac{\phi_{1}+\phi_{\mathrm{u}}}{2} \leqslant 1 \quad \text { and } \quad \phi=\frac{\phi_{1}-\phi_{\mathrm{u}}}{2} .
$$

This reparametrization is shown in Fig 3.3, where the red line in the left plot is the locus of all configurations whose average composition equals $\psi=1 / 2$ (shown in red in the right plot). Note that among all configurations compatible with such constraint, there are only two that extremize $\phi:\left(\phi_{\mathrm{u}}=1, \phi_{1}=0\right)$ and $\left(\phi_{\mathfrak{u}}=0, \phi_{1}=1\right)$, yielding $\phi=-1 / 2$ and $\phi=1 / 2$, respectively. The corresponding extrema for different values of $\psi$ is given by $\phi_{S \pm}= \pm(1-|\psi|)$. These will be referred to as the saturation values of the corresponding composition-difference subdomain.

Rewriting the free energy of mixing in terms of these new variables and taking into account the aforementioned curvature dependence via the number

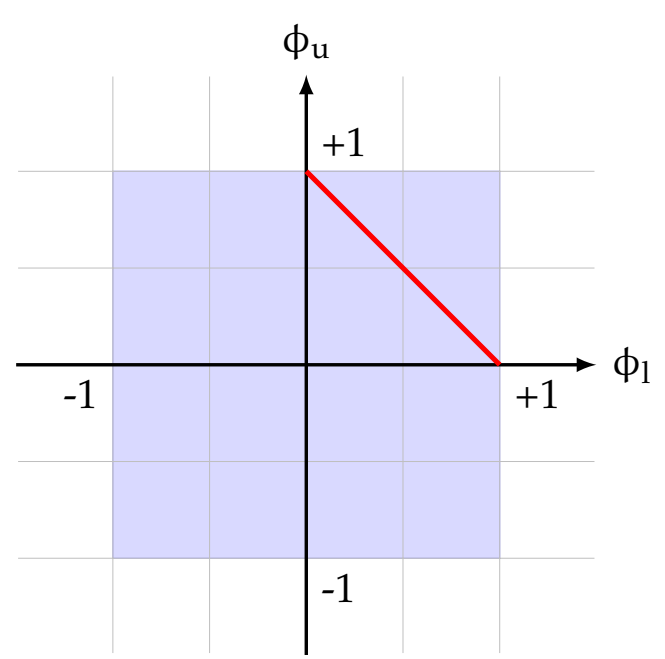

(a) Composition domain in the singlemonolayers representation, $\left\{\phi_{\mathrm{l}}, \phi_{\mathrm{u}}\right\}$.

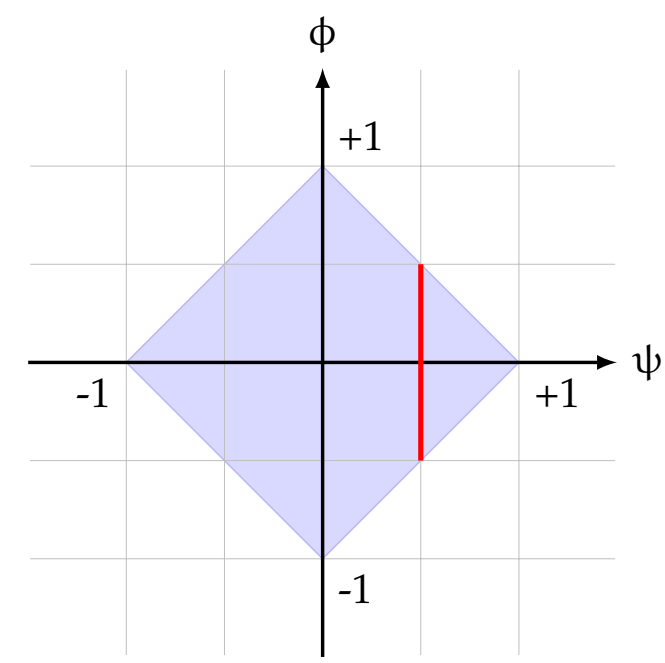

(b) Composition domain in the jointmonolayers representation, $\{\psi, \phi\}$.

Figure 3.3: Composition domain in the single- and joint-monolayers representations. The red line on the right is the plot of the red line on left, under the mapping Eq.3.17. 
of lipids per monolayer, results in the following expression:

$$
\frac{F_{m i x}}{\sigma_{m} A k_{B} T}=S_{1}+S_{u}+\frac{\chi}{2}\left(1-\psi^{2}-\phi^{2}\right)+\frac{t_{h}}{2} H\left(S_{1}-S_{u}-\chi \psi \phi\right),
$$

where the last term accounts for corrections due to the curvature-induced dilution or enhancement of interactions which, in turn, modify the monolayers density. In this expression, the entropic contributions from the upper and lower leaflets have been abbreviated as:

$$
\begin{gathered}
S_{\mathrm{u}}=\frac{1+\psi-\phi}{2} \ln \left(\frac{1+\psi-\phi}{2}\right)+\frac{1-\psi+\phi}{2} \ln \left(\frac{1-\psi+\phi}{2}\right), \\
S_{1}=\frac{1+\psi+\phi}{2} \ln \left(\frac{1+\psi+\phi}{2}\right)+\frac{1-\psi-\phi}{2} \ln \left(\frac{1-\psi-\phi}{2}\right) .
\end{gathered}
$$

\section{Curvature expansion and the equilibrium composition}

To rewrite the elastic energy in the new parametrization, first we perform an expansion to leading-order terms in curvature, so that Eq.3.13 reduces to:

$$
\frac{F_{b e n}}{k_{B} T}=\frac{\kappa_{m}}{2} A\left(C_{1}^{2}+C_{u}^{2}\right)+\frac{\kappa_{m}}{4} A t_{h} H\left(C_{1}^{2}-C_{u}^{2}\right)+\kappa_{m} A H\left(C_{1}+C_{u}\right) .
$$

Then, using the definition of the spontaneous curvature of a single monolayer and its coupling to composition, Eq 3.14. we can evaluate

$$
\begin{aligned}
& C_{1}-C_{u}=2 \eta_{\mathrm{m}} \lambda \phi, \\
& C_{1}^{2}-C_{u}^{2}=4 \eta_{\mathrm{m}} \lambda \phi\left(C_{0}+\eta_{\mathrm{m}} \lambda \psi\right), \\
& C_{1}^{2}+C_{u}^{2}=2 C_{0}^{2}+4 C_{0} \eta_{m} \lambda \psi+2 \eta_{m}^{2} \lambda^{2}\left(\psi^{2}+\phi^{2}\right) .
\end{aligned}
$$

Plugging these relations back into Eq 3.21 and retaining terms that depend on $\mathrm{H}$ and $\phi$ only, we obtain:

$$
\frac{F_{\text {ben }}}{\sigma_{m} A k_{B} T}=t_{h} H \phi\left(C_{0} \lambda+\eta_{m} \lambda^{2} \psi\right)+2 \lambda H \phi+\eta_{m} \lambda^{2} \phi^{2} .
$$

Note that the very last term of this expression can be incorporated into the definition of the bare $x$ parameter in $\mathrm{Eq} 3.18$, defining the effective incompatibility coefficient $\chi_{\text {eff }}=\chi-2 \eta_{\mathrm{m}} \lambda^{2}$.

Putting together the contributions from the elastic energy, Eq 3.22 , and the free energy of mixing, Eq 3.18, we obtain the final expression for the free energy 
of the bilayer:

$$
\begin{aligned}
\frac{F}{\sigma_{\mathrm{m}} A k_{\mathrm{B}} T}= & S_{1}+S_{\mathrm{u}}+\frac{\chi_{\text {eff }}}{2}\left(1-\psi^{2}-\phi^{2}\right)+\frac{t_{\mathrm{h}}}{2} H\left(S_{1}-S_{u}-\chi_{\text {eff }} \psi \phi\right) \\
& +t_{h} H \phi\left(C_{0} \lambda+\eta_{\mathrm{m}} \lambda^{2} \psi\right)+2 \lambda H \phi .
\end{aligned}
$$

Minimizing this expression with respect to $\phi$, to find the equilibrium composition difference, $\phi_{\text {eq }}$, for a given average composition and curvature (therefore the irrelevance of dropping constant terms as well as those that only depend on $\psi$ when deriving Eq.3.22, yields the following implicit relation:

$$
\begin{aligned}
\frac{1}{2} \ln \left[\frac{\left(1+\phi_{\mathrm{eq}}\right)^{2}-\psi^{2}}{\left(1-\phi_{\mathrm{eq}}\right)^{2}-\psi^{2}}\right] & +\frac{t_{\mathrm{h}} \mathrm{H}}{4} \ln \left[\frac{(1+\psi)^{2}-\phi_{\mathrm{eq}}^{2}}{(1-\psi)^{2}-\phi_{\mathrm{eq}}^{2}}\right]= \\
& \chi_{\mathrm{eff}} \phi+\frac{1}{2} \chi_{\mathrm{eff}} \mathrm{t}_{\mathrm{h}} \mathrm{H} \psi-\mathrm{t}_{\mathrm{h}} \mathrm{H}\left(\mathrm{C}_{0} \lambda+\eta_{\mathrm{m}} \lambda^{2} \psi\right)-2 \lambda H
\end{aligned}
$$

For a fixed average composition, $\psi$, this expression relates the composition asymmetry between leaflets to the midplane curvature, therefore quantifying the efficiency of the lipid-sorting mechanism. The graph of this expression for different $\psi$-level curves is shown in Fig.3.4. These curves correspond to a set of parameters compatible with the previous estimation of the implicit coupling and, additionally, consider a system in the vicinity of the mean-field demixing critical point, $\chi_{\mathrm{c}}=2$.

An important feature to point out from these plots is the asymptotic approach towards the corresponding saturation value, $\phi_{S_{ \pm}}= \pm(1-|\psi|)$, as the curvature increases. This saturation emerges when the local packing of lipids becomes highly constrained. To elucidate the development of such a frustrated state, a simple geometrical picture, appealing to the spontaneous curvature of the individual lipid species, may be helpful: if these individual curvatures are incommensurable, in the sense that their corresponding radius of curvature do not differ exactly by one bilayer thickness (lets say $R_{1}=R$ and $R_{2}=R+\delta$, with $\delta>t_{h}$ ), it will be impossible (arguing on geometrical grounds only) to assembly those lipids into vesicles or cylinders whose midplane radius, $R_{v}$, equals the average radius between the two species. If it were possible, such an ideal system would necessarily be characterized by $\psi=0$ and $\phi_{\text {eq }}=\phi_{\mathrm{S}}=1$, i.e., a membrane with completely opposite composition on its apposing leaflets, as shown in Fig 3.5a. Furthermore, this saturation state will be characterized by a finite midplane curvature, $C_{v}=1 / R_{v}=2 /\left(2 R+t_{h}\right)$. When these curvatures are incommensurate, a complete phase separation across leaflets would inevitably result in the presence of interstices. These would induce a high tension in the membrane due to the concomitant hydrophobic density mismatch or 


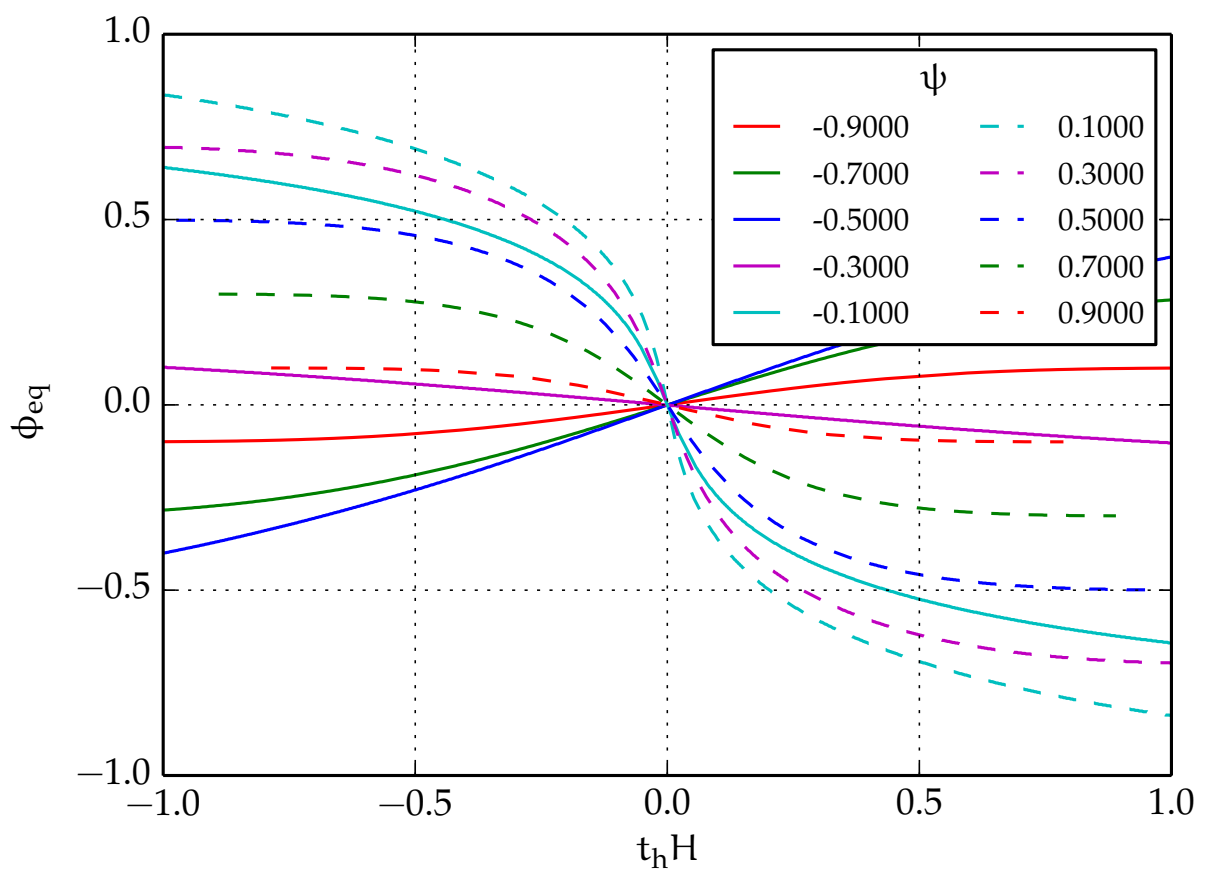

Figure 3.4: Solutions to the implicit relation, Eq.3.24, between the composition difference, $\phi$, and curvature, $H$, for $t_{h}=6.0 \mathrm{~nm}, C_{0}=-0.1 \overline{3} \mathrm{~nm}^{-1}$, $\chi_{\text {eff }}=1.9$, $\eta_{\mathrm{m}}=0.2 \mathrm{~nm}^{-2}, \lambda=3.0 \mathrm{~nm}$ and different $\psi$-level curves.

the exposure of the lipid tails to the aqueous environment, factors that will ultimately compromise the bilayer integrity. To avoid these situations, some lipids will migrate to the "wrong" leaflet to fill the interstices, therefore allowing the formation of highly-curved structures, though at the expense of a deteriorated saturation state, as shown in Fig $3.5 b$.

Viewed from the perspective of low curvatures, flat membranes can only accommodate a completely symmetric composition on each monolayer, i.e., $\psi=0$ and $\phi_{\text {eq }}=0$. Having zero curvature, they can be thought as infinitely extended objects, composed by an infinite number of lipids. As this flat surface starts to bend, lipids will have to be exchanged between monolayers to prevent the formation of interstices, therefore inducing a composition asymmetry between leaflets. This lipid exchange does not represent a problem for small curvatures, since there will be many places to put these insertions, resulting in the same curvature. It is therefore reasonable to expect a linear dependence of the composition difference on curvature. However, as the saturation point is reached, the space to accommodate new insertions will become scarce and the system may prefer to perform local reorganizations within the individual monolay- 


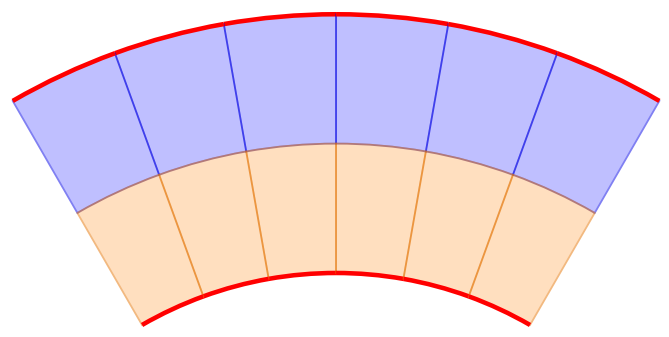

(a) Commensurate curvatures.

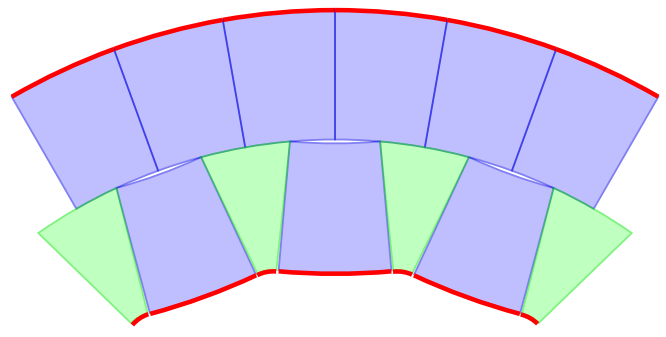

(b) Incommensurate curvatures.

Figure 3.5: Assembly of lipids with commensurate and incommensurate curvatures. The red edges represent the location of the polar head-groups for each species.

ers, which still contribute to increasing the composition difference, though in a less efficient manner, until the scenario described in the previous paragraph is finally reached. Of course, curvatures beyond this scenario have no physical meaning, at least within this simplified description where lipids are not allowed to modify their shape to adapt to their environment. In the following this will be denoted as the saturation curvature.

\section{The effective curvature-composition coupling, $\wedge_{\text {eff }}$}

Another interesting feature observed in the curves shown in Fig.3.4, is that the speed at which the saturation regime is reached, depends on $\psi$. Qualitatively this can be understood as follows: shifting the average composition shifts the saturation value, i.e., the maximum composition asymmetry across the leaflets. This will inevitably modify the ability of the system to accommodate new lipid flip-flops and, furthermore, will also shift the maximum attainable curvature before the appearance of interstices becomes unavoidable (i.e., the region where the logarithmic damping becomes important). Of course, the geometrical picture used so far is not enough to provide a quantitative description, since the way the lipid insertions are constrained will further depend on how prone are the lipid species to mix. Fortunately, such a quantitative analysis can be done in an analytic way, at least for composition differences smaller than the corresponding saturation value, because in that limit Eq 3.24 reduces to an explicit relation, $\phi_{\text {eq }}=\Lambda_{\text {eff }} \mathrm{H}$, where $\Lambda_{\text {eff }}$ is the $\psi$-dependent, effective curvature-composition coupling:

$$
\Lambda_{\text {eff }}=\frac{\frac{1}{2} \ln \left(\frac{1+\psi}{1-\psi}\right)+\psi\left(\eta_{\mathrm{m}} \lambda^{2}-\frac{1}{2} \chi_{\mathrm{eff}}\right)+\lambda\left(\frac{2}{\mathrm{t}_{\mathrm{h}}}+\mathrm{C}_{0}\right)}{\chi_{\mathrm{eff}}-\frac{2}{1-\psi^{2}}} .
$$


The first term in the numerator is nothing but the contribution from the previously described geometrical picture. Note that for systems with almost equal compositions for both lipid species, i.e., $\psi \approx 0$, this contribution is small, meaning that the saturation composition difference is to be reached at high curvatures. Furthermore, in the small- $\psi$ regime, it scales linearly, reflecting the large capability of the system to accommodate new flip-flops. On the contrary, when the system favors the presence of a single species, this contribution diverges logarithmically, showing that the saturation will be reached at low curvatures, i.e., almost flat configurations. Thus, this contribution is in complete agreement with the previous (qualitative) discussion.

The second term is a correction to the entropic sorting that beside accounting for the elastic properties of the system (the bending rigidity, $\mathrm{K}_{\mathrm{m}}=\sigma_{\mathrm{m}} / \eta_{\mathrm{m}}$ and via the intrinsic curvature-composition coupling, $\lambda$ ), also incorporates the propensity of the two species to mix. In general, a high segregation would strongly reduce the ability of the monolayers to accommodate new insertions, therefore shifting the saturation regime towards smaller curvatures than the ones attained in the low incompatibility counterpart, i.e., we would expect the presence of large effective couplings for strong segregation conditions.

The last term in the numerator is a constant that quantifies the saturation curvature, though apart from the purely geometrical argument previously discussed, it is re-scaled by the intrinsic coupling, which could account for deviations from the ideal case, due to the incommensurability of the two species.

Finally, the denominator of this expression turns out to be the inverse susceptibility of composition fluctuations. This quantity vanishes as the system approaches the mean-field critical point, $\chi_{c}=2$, therefore enhancing the effective curvature-composition coupling.

The effective curvature-composition coupling, as a function of the average composition, is shown in Fig 3.6. It is plotted for different incompatibilities and with the same set of parameters than those used in Fig 3.4 . These plots exhibit the enormous curvature modulation for systems in the close vicinity of the demixing critical point. Another interesting aspect is the lack of symmetry with respect to the $\psi$ and $\Lambda_{\text {eff }}$ axes. The former, is a direct consequences of the constant term in the numerator of Eq 3.25 . It reflects the natural tendency of the system to favor the proper sorting of lipids, based exclusively on their geometry. On the contrary, the fact that $\Lambda_{\text {eff }}$ becomes positive for certain compositions $(\psi \lesssim-0.3$ in this case, given the sign conventions for curvature and composition) indicates that the system may prefer to accommodate lipids in such a way that the composition of apposing leaflets is contrary to what would be expected from the purely geometrical picture. However, as we have already seen, even within the geometrical picture, the migration of lipids into curvature- 


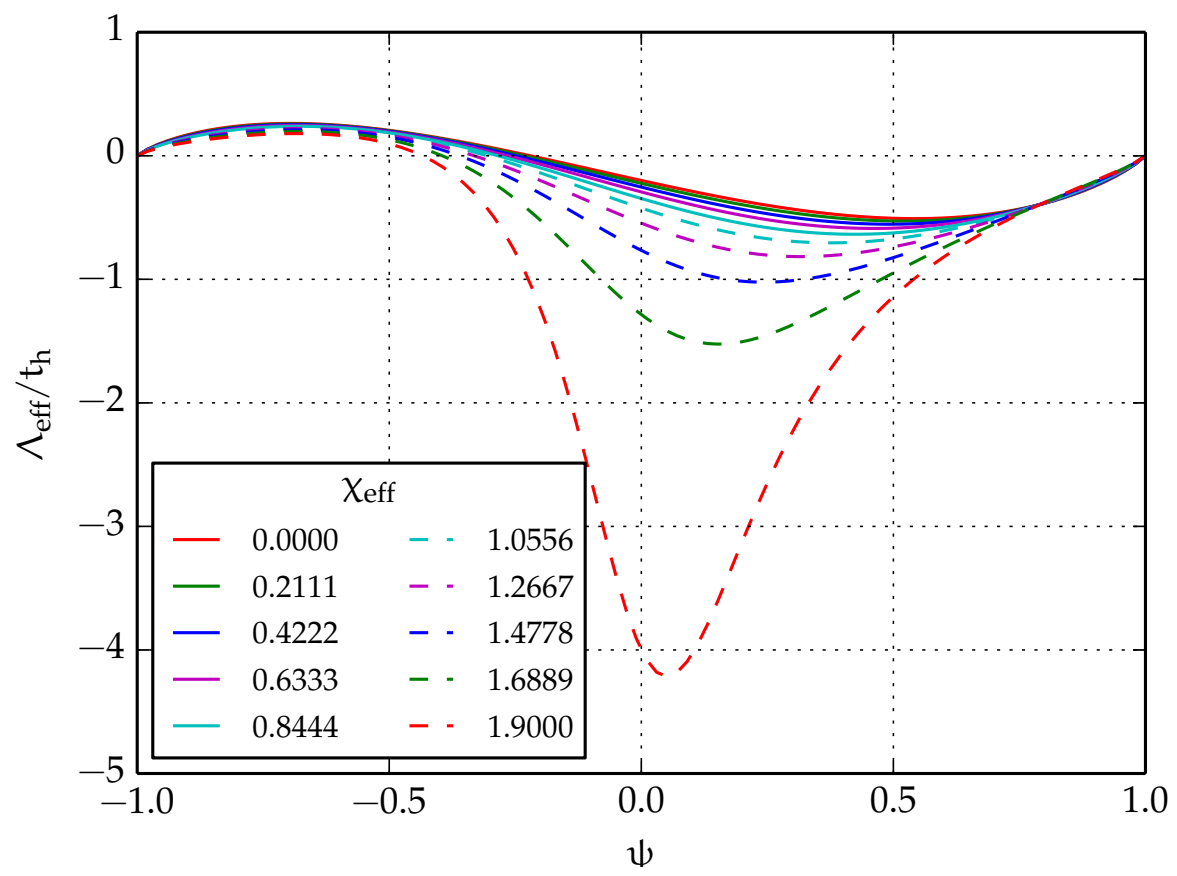

Figure 3.6: Effective coupling, $\wedge_{\text {eff }}$, between composition difference and curvature, as a function of average compositions, $\psi$, for different incompatibilities, $\chi_{\text {eff. }}$ The rest of parameters for these curves are $t_{h}=6.0 \mathrm{~nm}, C_{0}=-0.1 \overline{3} \mathrm{~nm}^{-1}$, $\eta_{\mathrm{m}}=0.2 \mathrm{~nm}^{-2}, \lambda=3.0 \mathrm{~nm}$.

unfavorable regions may be possible. Therefore, it should be expected (or perhaps not very surprising) that the inclusion of additional interactions, further enhances this phenomenon, at least within a low-curvature regime.

\subsection{Curvature-dependent bending rigidity}

In the previous section we introduced a phenomenological model to study the interplay between curvature and composition in lipid bilayers. The novelty of this model is that it explicitly takes into account the difference of curvature between the two leaflets of the membrane. However, in this model the bending rigidity of these monolayers is a constant, independent of curvature. This assumption seems to be reasonable for systems in the presence of small deformations, such as those typically induced by thermal fluctuations or for closed bilayer structures, such as vesicles or membrane tubes, whose radius of curvature is considerably larger than the bilayer thickness, $t_{h}$. However, as curva- 
tures become comparable to $t_{\mathrm{h}}^{-1}$, the presence of packing effects may ultimately invalidate this assumption. ${ }^{[30}$ In this section, we further extend our model to incorporate the high-curvature contributions to the bending rigidity. However, to facilitate the analysis, we will still assume that this dependence arises from the curvature of the bilayer midplane. Therefore, the bending modulus of both monolayers remains the same. Explicitly, we will consider:

$$
\kappa_{\mathrm{u}}=\kappa_{1}=\kappa_{\mathrm{m}}\left(1+\frac{\kappa_{1}}{2} \mathrm{H}^{2}\right) .
$$

Plugging this expression into the definition of the elastic energy of the bilayer, $\mathrm{Eq}$ 3.13, we obtain:

$$
\begin{aligned}
\frac{F_{\text {ben }}}{k_{B} T}= & \frac{\kappa_{u}}{2} A_{u}\left\{H_{u}-\left[C_{0}+\left(\frac{\sigma_{m}}{\kappa_{u}} \lambda\right) \phi_{u}\right]\right\}^{2} \\
& +\frac{\kappa_{l}}{2} A_{1}\left\{H_{1}-\left[C_{0}+\left(\frac{\sigma_{m}}{\kappa_{l}} \lambda\right) \phi_{1}\right]\right\}^{2} .
\end{aligned}
$$

Using the relations for the area and curvature of each monolayer, in terms of the corresponding quantities for the bilayer midplane, Eqs. 3.11 and 3.10, expanding up to second-order terms in $\mathrm{H}$ and retaining terms that include compositiondependent prefactor only, we get the following contribution for the elastic energy of the membrane:

$$
\begin{aligned}
\frac{F_{\text {ben }}}{\sigma_{\mathrm{m}} A k_{\mathrm{B}} T}= & \mathrm{t}_{\mathrm{h}} \mathrm{H} \phi\left(\mathrm{C}_{0} \lambda+\eta_{\mathrm{m}} \lambda^{2} \psi\right)+2 \lambda H \phi+\eta_{\mathrm{m}} \lambda^{2} \phi^{2} \\
& -\frac{1}{2}\left(\frac{\mathrm{k}_{1} \eta_{\mathrm{m}} \lambda^{2}}{\mathrm{t}_{\mathrm{h}}^{2}}\right) \phi^{2} \mathrm{t}_{\mathrm{h}}^{2} \mathrm{H}^{2} .
\end{aligned}
$$

The only difference between this expression and the corresponding linear expansion, Eq3.22, is the very last term, which vanishes for $\kappa_{1}=0$. With these $\mathrm{H}^{2}$-corrections, the implicit relation between the composition difference and curvature reads:

$$
\begin{aligned}
\frac{1}{2} \ln \left[\frac{\left(1+\phi_{\text {eq }}\right)^{2}-\psi^{2}}{\left(1-\phi_{\text {eq }}\right)^{2}-\psi^{2}}\right]+\frac{t_{h} H}{4} \ln \left[\frac{(1+\psi)^{2}-\phi_{\text {eq }}^{2}}{(1-\psi)^{2}-\phi_{\text {eq }}^{2}}\right]= \\
\chi_{\text {eff }} \phi+\frac{1}{2} \chi_{\text {eff }} t_{h} H \psi-t_{h} H\left(C_{0} \lambda+\eta_{\mathrm{m}} \lambda^{2} \psi\right)-2 \lambda H \\
+\left(\frac{\kappa_{1} \eta_{\mathrm{m}} \lambda^{2}}{t_{h}^{2}}\right) \phi_{\text {eq }} t_{h}^{2} H^{2}
\end{aligned}
$$


Unfortunately, this expression does not become explicit (in general) in the far from saturation limit. Different $\mathrm{k}_{1}$-level curves of this function are shown in Fig 3.7. These plots stand for a system with a symmetric composition of lipids, $\psi=0$, and high incompatibility, $\chi_{\text {eff }}=1.9$, conditions under which the largest effect is expected. The chosen values of $k_{1}$ (measured in units of $k_{B} T_{c}^{2}$ ) have intentionally been exaggerated, with respect to those reported in the literature (typical values are below $\left.2.0 \mathrm{k}_{\mathrm{B}} \mathrm{Tnm}^{2}\right),{ }^{20}$ with the intention to exhibit the small effect of these $\mathrm{H}^{2}$ contributions, therefore justifying the use if the simpler linear expansion for the analysis of our simulations.

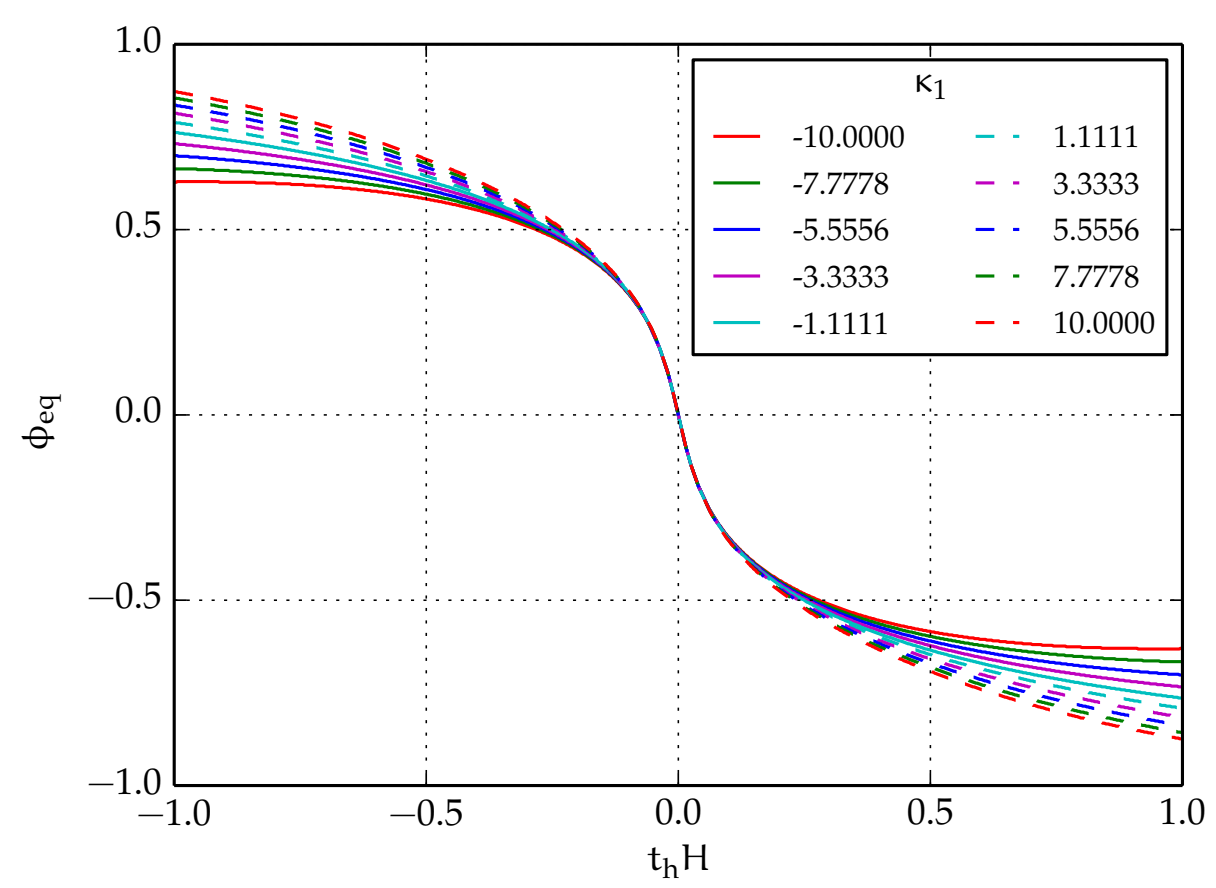

Figure 3.7: $\mathrm{H}^{2}$ corrections to the implicit relation between composition difference and curvature, Eq. 3.29 , for $\psi=0, t_{\mathrm{h}}=6.0 \mathrm{~nm}, \mathrm{C}_{0}=-0.1 \overline{3} \mathrm{~nm}^{-1}$, $\chi_{\text {eff }}=1.9$, $\eta_{\mathrm{m}}=0.2 \mathrm{~nm}^{-2}, \lambda=3.0 \mathrm{~nm}$ and different $\mathrm{k}_{1}$-level curves.

A final remark for the role of these contributions is that, when the membrane becomes less rigid, i.e., $\mathrm{k}_{1}<0$, saturation is reached faster. This is in complete agreement with the geometrical picture, because an easy-bending leaflet will have an increased capability to accommodate new insertions, thus further promoting the lipid sorting. 


\section{Chapter 4}

\section{Interplay between curvature and composition in lipid bilayers}

In the previous chapter we introduced a phenomenological model that describes the thermodynamics of mixing in bilayer membranes composed of lipids with different spontaneous curvatures. The novelty of this model is that it transfers the bilayer structure of the membrane not only to the composition or density field, but also to the local curvature. We pointed out that this consideration captures important saturation effects that emerge when local shape modulations are comparable to the bilayer thickness. In this chapter, we perform molecular dynamics simulations of lipid bilayers with different geometries (curvature regimes) to validate the predictions of the aforementioned mean-field model. These simulations are carried out within the implicit-solvent, coarse-grained model introduced in Chapter 2.

In the first section, we provide an overview of previous works that helped us to parametrize the two lipid species used in the simulations. There, we also present the measurements of certain mechanical properties of one-component bilayers and provide a first insight on the intrinsic curvature of the lipid species. In the second section, we continue the study of the elastic and thermodynamical properties of pure and mixed bilayers and, based on those studies, we determine most of the coefficients in our mean-field model. The third section concerns itself with the study of the correlation between the local curvature and the composition asymmetry across the bilayer, in planar membranes far from saturation. Under these conditions, it is verified that $\phi_{\mathrm{eq}}$ and $\mathrm{H}$ are linearly related, via the effective curvature-composition coupling. Finally, in the fourth section, we investigate the lateral sorting of lipids in systems where the farfrom-saturation conditions are not met. For those cases, it is verified that the implicit relation between curvature and composition, Eq.3.24, duly accounts for saturation stemming from packing effects. 


\subsection{Simulation setup}

The coarse-grained model introduced in the Chapter 2 has proved to reproduce the rich phase diagram of biologically relevant lipids. In a previous work, Hömberg and Müller ${ }^{36 / 82}$ have carried out a systematic study of the parameter space and lipid architectures leading to the self-assembly of different morphologies. These morphologies include: i) micelles, characterized by the presence of a single head-group interface, which encapsulates the hydrophobic interior. These micelles are usually composed of convex, single-tailed lipids, where the volume fraction of the polar head-group is larger than that of the hydrophobic tail. Depending on the curvature of the individual lipid species, micelles can self-assemble into spherical, cylindrical or wormlike geometries. ii) Inverted morphologies, also characterized by a single interface. In contrast to micelles, the head-group interface of these inverted structures is bent in the direction of the outer normal (concave deformations). These morphologies are observed for lipids whose tail volume-fraction is considerably larger than that of their headgroup. Finally, the model also exhibits the self-assemble of iii) bilayer structures, where the hydrophobic tails of two apposing monolayers come into close contact, leaving the polar head-groups facing the "vacuum solvent", preventing the exposure of the hydrophobic interior. These morphologies are preferably observed for "cylindrical" lipids, where the difference in volume-fraction be-

Table 4.1: Self-assembled morphologies obtained from a random initial configuration. The column headers on the right denote the molecular asymmetry, e.g., 10/6 means $N_{A}=10$ and $N_{B}=6$. The morphologies are abbreviated: $\mathrm{s}=$ spherical micelles, $\mathrm{c}=$ cylindrical micelles, $\mathrm{w}=$ wormlike micelles, $\mathrm{b}=$ bilayers, $\mathrm{i}=$ inverted structures, i.e., bilayers with hydrophilic inclusions.

\begin{tabular}{cccc|cccccc}
\hline \hline$\rho_{\text {coex }}$ & $\mathrm{kN}$ & $\chi \mathrm{N}$ & $\mathrm{k}_{\mathrm{b}}$ & $10 / 6$ & $11 / 5$ & $12 / 4$ & $13 / 3$ & $14 / 2$ & $15 / 1$ \\
\hline 15 & 100 & 40 & 0 & $\mathrm{c}$ & $\mathrm{b}$ & $\mathrm{b}$ & $\mathrm{i}$ & $\mathrm{i}$ & $\mathrm{i}$ \\
15 & 100 & 40 & 5 & $\mathrm{~s}$ & $\mathrm{~b}$ & $\mathrm{~b}$ & $\mathrm{~b}$ & $\mathrm{i}$ & $\mathrm{i}$ \\
15 & 100 & 60 & 0 & $\mathrm{~s}$ & $\mathrm{~b}$ & $\mathrm{~b}$ & $\mathrm{~b}$ & $\mathrm{i}$ & $\mathrm{i}$ \\
15 & 100 & 60 & 5 & $\mathrm{~s}$ & $\mathrm{~b}$ & $\mathrm{~b}$ & $\mathrm{i}$ & $\mathrm{i}$ & $\mathrm{i}$ \\
18 & 80 & 20 & 5 & $\mathrm{~s}$ & $\mathrm{w}$ & $\mathrm{w}$ & & & \\
18 & 80 & 30 & 5 & $\mathrm{~s}$ & $\mathrm{c}$ & $\mathrm{w}$ & & & \\
18 & 80 & 40 & 5 & $\mathrm{~s}$ & $\mathrm{c}$ & $\mathrm{w}$ & & & \\
20 & 100 & 20 & 5 & $\mathrm{~s}$ & $\mathrm{c}$ & $\mathrm{b}$ & $\mathrm{b}$ & & \\
20 & 100 & 30 & 5 & $\mathrm{~s}$ & $\mathrm{c}$ & $\mathrm{b}$ & $\mathrm{i}$ & & \\
20 & 100 & 40 & 5 & $\mathrm{~s}$ & $\mathrm{~s}$ & $\mathrm{c}$ & $\mathrm{b}$ & & \\
20 & 100 & 50 & 5 & $\mathrm{~s}$ & $\mathrm{~s}$ & $\mathrm{w}$ & $\mathrm{b}$ & & \\
\hline \hline
\end{tabular}


tween the hydrophobic and hydrophilic blocks is not as large as that of micelles or inverted structures. A comprehensive list of the different parameters, chain architectures and the resulting morphologies, studied by Hömberg et al., is provided in Tab 4.1

It is worth mentioning that beside the self-assembly of the different morphologies reported in Tab4.1, a more detailed study of the parameter space has shown that the model is also able to reproduce the full phase diagram of bilayer structures. Specifically, the fluid $\left(\mathrm{L}_{\alpha}\right)$, gel $\left(\mathrm{L}_{\beta}\right)$, interdigitated gel $\left(\mathrm{L}_{\beta \mathrm{I}}\right)$ and gel-tilted $\left(\mathrm{L}_{\beta^{\prime}}\right)$ phases have been observed. Non-surprisingly, the strength of the bond-angle bending potential, $\mathrm{k}_{\mathrm{b}}$, and the inverse compressibility, $\mathrm{kN}$, have a large influence on the emergence of the gel phases. If $k_{b}=0$, chains will become completely flexible and their conformations will resemble those of a self-repelling random walk, where shape fluctuations are comparable to the lipid extension, $R_{\mathrm{eo}}$. Conversely, if $k_{\mathrm{b}}$ is large, the orientation of successive beads will be highly correlated. This alignment, together with the presence of the polar head-group interfaces, will induce an smectic liquid-crystal ordering of the lipids in the two monolayers.

Starting from a liquid bilayer (which in any case should exhibit certain degree of orientational correlation), a similar transition towards the gel phase can be achieved by increasing the inverse compressibility. In that case, the individual beads will become more rigid and will need to rearrange in order to avoid overlaps with neighboring beads. Relocations along the bilayer plane, however, will be avoided due to the concomitant exposure of the hydrophobic interior. Therefore, beads will shift along the membrane normal, effectively enhancing the orientational correlation along the chain and eventually crossing the border between the fluid and the gel phases.

Based on these studies, we have selected the pair of lipid architectures and set of parameters shown in Tab, 4.2, to study the interplay between curvature and lipid composition, described by our phenomenological expression for the effective curvature-composition coupling, $\Lambda_{\text {eff. }}$. According to the results of Hömberg et al., the first of these lipids (which from now on will be referred to as Btype or short head-group species) should self-assemble into fluid bilayers and the second architecture (which we will denote as C-type or large head-group species), should lie in the border between micelles and bilayers. Starting from a completely random distribution of lipids within the simulation box, we have verified that both species self-assemble into flat bilayer structures, as shown in the snapshots in Fig. 4.1 


\section{CHAPTER 4. INTERPLAY BETWEEN CURVATURE AND COMPOSITION IN LIPID BILAYERS}

Table 4.2: Set of parameters used in this work for the study of the curvaturecomposition coupling, $\Lambda_{\text {eff }}$.

\begin{tabular}{|c|c|c|}
\hline Head-type & Tail-type & $\mathrm{N}_{\text {head }}$ \\
\hline B & $\mathrm{A}$ & 4 \\
\hline C & A & 5 \\
\hline
\end{tabular}

(a) Short head-group lipid

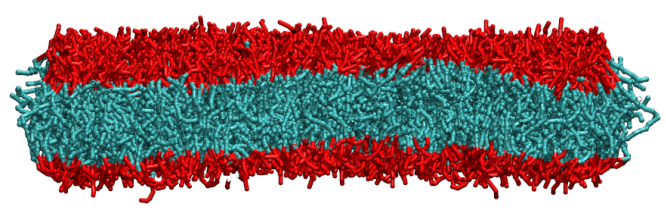

(b) Large head-group lipid

Figure 4.1: Self-assembly of B- and C-type lipids (with short and large headgroups, respectively) into planar bilayers.

\subsubsection{Area per lipid}

An important assumption made when deriving the mean-field theory for the effective curvature-composition coupling, Eq 3.23, was the independence of the areal density on curvature and composition. The main contribution to the composition dependence is due to the difference in area per lipid between the two species, 138 although higher-order contributions may arise, due to interactions between unlike species. To determine the area per lipid, we have performed simulations of pure component systems in the $\mathrm{NP}_{\mathrm{t}} \mathrm{T}$ ensemble at zero tension. From these simulations, the area per lipid was obtained after averaging the area fluctuations of the simulation box and dividing by the total number of lipids on each monolayer (in this case, we simulated a total of 4680 lipids, i.e., 2340 lipids per leaflet on average). We have found that the area per lipid of the two chain architectures differs in less than $1 \%$, being the average value of the two measurements $\langle a\rangle=0.565 \pm 0.003 r_{\mathrm{c}}^{2}$. At first, this seems a contra-intuitive result, since one would expect that the species with the larger head-group span over a broader area. However, since the total number of beads per chain, $\mathrm{N}$, is kept fixed, the bulky tail would be effectively reduced as the length of the headgroup increases. It is the combined effect of these two, opposite behaviors that leaves the area of these $\mathrm{N}$-fixed chain architectures unchanged. However, equal areas per lipid does not imply equal curvatures. The total volume dictates the area per lipid, but it is the difference in volume fractions between the headgroup and tail blocks that determines the geometry of the corresponding lipid architecture. ${ }^{19} \mathrm{~A}$ first indicator of the geometry changes induced when modifying the relative length of the two blocks, might be grasped by considering the 
stress distribution across the bilayer.

\subsubsection{Area compressibility}

Simulations in the $\mathrm{NP}_{\mathrm{t}} \mathrm{T}$ ensemble were also useful to evaluate the compressibility modulus of the bilayer, $\kappa_{A}$, which quantifies the resistance of the system to sustain area deformations and is defined by

$$
\kappa_{A} \equiv A \frac{\partial \gamma}{\partial A}
$$

where $\gamma$ is the surface tension of the system. In the limit of small deformations with respect to the equilibrium area at zero tension, $A_{0}$, this expression may be approximated as

$$
\gamma=\kappa_{\mathrm{A}}\left(\frac{\mathrm{A}-\mathrm{A}_{0}}{\mathrm{~A}_{0}}\right)
$$

Therefore, the leading-order term of the tensile deformations contribution to the free energy of the bilayer will be quadratic,

$$
F=\frac{\kappa_{A}}{2 A_{0}}\left(A-A_{0}\right)^{2} .
$$

Within this approximation, the distribution of areas in the $N P_{t} T$ ensemble will be Gaussian,

$$
P(A)=\frac{1}{z} e^{-F(A) / k_{B} T} \propto \exp \left[-\frac{\kappa_{A}}{2 A_{0} k_{B} T}\left(A-A_{0}\right)^{2}\right],
$$

with mean value equal to $\langle A\rangle=A_{0}$ and a variance inversely proportional to the compressibility modulus, $\sigma_{A}^{2}=A_{0} k_{B} T / \kappa_{A}$. This yields the well-known relation between the susceptibility of area fluctuations and the compressibility,

$$
\kappa_{A}=k_{B} T \frac{\langle A\rangle}{\left\langle A^{2}\right\rangle-\langle A\rangle^{2}}
$$

Using this expression, we have found the compressibility of the short and large head-group lipids to be $\mathrm{K}_{A}=31.97$ and $\mathrm{K}_{A}=33.70$, respectively. From these, it is possible to obtain a first (although crude) estimation of the corresponding bending rigidities, since $\mathrm{k}$ and $\mathrm{K}_{\mathrm{A}}$ are related via the following equation $119[139 \mid 140$

$$
\kappa=\frac{1}{48} \kappa_{A} t_{h}^{2}
$$

Anticipating that our measurements of the bilayer thickness are composition independent and yield $t_{h}=6 r_{c}$, the estimated values of the bending rigidities, 


\section{CHAPTER 4. INTERPLAY BETWEEN CURVATURE AND COMPOSITION} IN LIPID BILAYERS

obtained from this expression for the short and large head-group lipid species are $k \approx 23.98$ and $k \approx 25.28$. Later, we will see that although slightly overestimating, these values agree with the results obtained from the spectra of shape fluctuation.

\subsubsection{Lateral pressure profile}

The lateral pressure profile along the bilayer normal ( $\hat{\mathbf{z}}$ in this case) is defined as the difference between the normal, $P_{N}=\mathbf{P}_{z z}$, and the lateral, $P_{L}=\left(\mathbf{P}_{x x}+\mathbf{P}_{y y}\right) / 2$, components of the pressure tensor, $\mathbf{P}$,

$$
\Gamma(z)=\mathrm{P}_{\mathrm{L}}(z)-\mathrm{P}_{\mathrm{N}}(z)
$$

The integral of $\Gamma(z)$ along the normal direction yields the surface tension of the system

$$
\gamma=\int_{-t_{h} / 2}^{t_{h} / 2} \mathrm{~d} z \Gamma(z),
$$

and its first and second integral momenta are related to the product of the bending rigidity and the spontaneous curvature of a single monolayer, $\mathrm{K}_{\mathrm{m}} \mathrm{C}_{0}$, and its splay modulus, $\overline{\mathrm{K}}_{\mathrm{m}}$, respectively. 141

The condition of local mechanical equilibrium, $\nabla \cdot \mathbf{P}=0$, for a planar membrane in the fluid state results in a diagonal tensor with the normal component equal to the bulk pressure of the system. Therefore, in this work, $\Gamma(z)$ reduces to the negative of lateral pressure, since $\mathrm{P}_{\mathrm{N}}=0$ for our solvent free model.

To evaluate the pressure profile from simulated data, we discretized the simulation box along the perpendicular direction to the membrane plane, into small slabs of thickness $\Delta z$ and volume $\Delta \mathrm{V}$. Then, taking advantage of the pairwise discretization of the forces in our model, Eqs.2.15, we computed the contribution to the pressure at each slab, from the following expression for the local virial, 2223

$$
\mathbf{P}_{\text {loc }}(z)=\sum_{i \in \text { slab }} m_{i} \mathbf{v}_{\mathfrak{i}} \otimes \mathbf{v}_{\mathfrak{i}}-\frac{1}{\Delta V} \sum_{i<j} \mathbf{F}_{\mathfrak{i j}} \otimes \mathbf{r}_{\mathfrak{i j}} f\left(z, z_{\mathfrak{i}}, z_{\mathfrak{j}}\right),
$$

where $\mathbf{m}, \mathbf{r}$ and $\mathbf{F}$ are, respectively, the particle mass, velocities and forces. Notice that the second sum in this expression is weighted by the function $f$, which sets the amount of virial to be added to the current slab. Following Lindhal and Edholm, 22 these weights were assigned as follows: if both particles $i$ and $j$ belong to the current slab, $f=1$. If both particles are outside the slab but it is intersected by the line passing their centers, then $f=\Delta z /\left|z_{i}-z_{j}\right|$, and set 


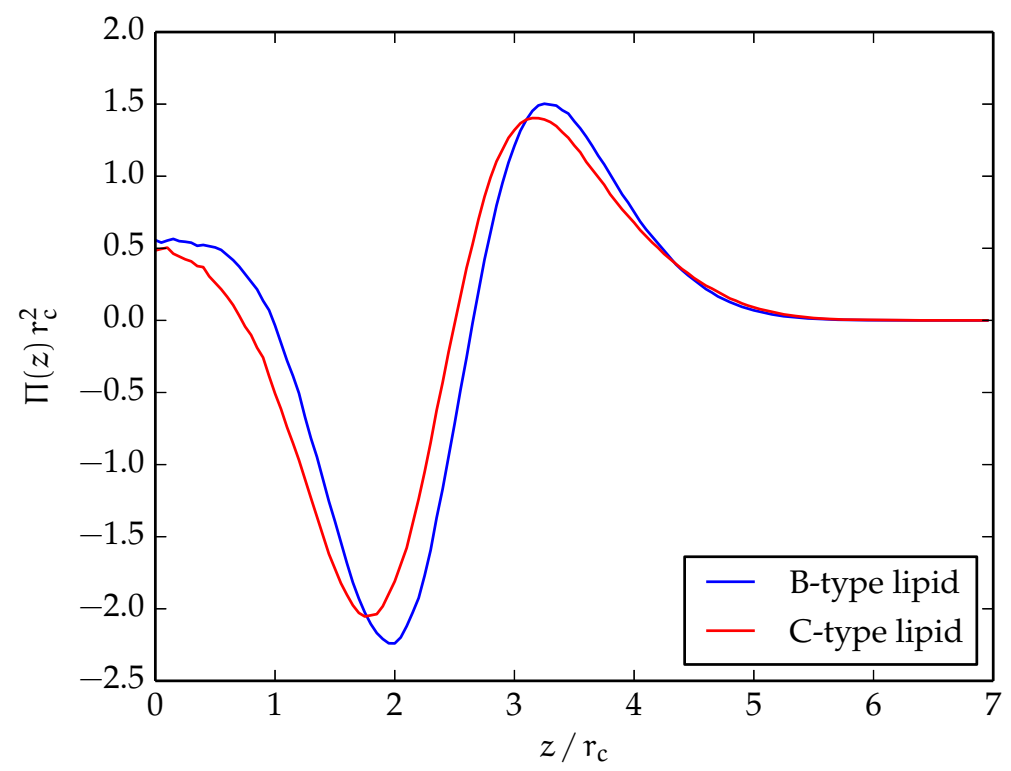

Figure 4.2: Pressure profile for the short (blue) and large (red) head-group species.

to zero otherwise. Finally, if one of the particles lies inside the box, we set $f=\Delta z / 2\left|z_{i}-z_{j}\right|$.

In Fig, 4.2 we present the pressure profiles obtained from our tensionless simulations for the short (blue) and large (red) head-group species. However, since for an homogeneous and flat membrane the profile is symmetric with respect to the bilayer midplane, only the half corresponding to the upper monolayer is shown. The pronounced pressure drop at $z \sim 2$ occurs exactly at the interface between the tail and head-group blocks. The resulting negative pressure (contractile force) is the response of the system trying to minimize the surface area of that interface. $\frac{141}{1}$ Meanwhile, within the bulk, although still in the vicinity of the interface, excluded volume interactions will come into play to counteract the effect of bringing the particles into close contact. Since the equation of state for the head-group beads has been set to good solvent conditions, this restoring effect will be enhanced within the hydrophilic region, hence the prominence of the peak at $z \sim 3$.

In accordance with this steric argument, the presence of a peak at $z=0$ will imply an increased density at the tail-tail interface. However, as we will see in a later section, the pressure profile is homogeneous all over the hydrophobic interior. Therefore, it is not feasible that the excluded volume interactions are responsible for this phenomenon. A more reasonable explanation associates this peak with the increased configurational entropy of the chain-ends, which 
are less constrained to bonds with neighboring beads. ${ }^{141}$ This reasoning, however, is still under debate and a more exhaustive analysis is in turn to extract a final conclusion. Furthermore, when compared to the pressure profiles from atomistic or systematically coarse-grained simulations, 15 22:142 our profiles lack a dip in the hydrophobic peak as $z \rightarrow 0$. This can be attributed to the absence of a similar dip in the density profile at the tail-tail interface.

Over the last decade, the pressure profile and its integral momenta have been extensively investigated by theoretical descriptions and computer simulations. One of the main reasons for that was to provide a way of quantifying the curvature of different lipid species. However, a drawback of this approach is that, up to now, the experimental determination of the pressure profile is not possible. Therefore the need of new strategies to evaluate the curvature of individual lipid species and, furthermore, that this techniques provide a direct method to compare measurements from computer simulations and experiments.

\subsection{Non-free parameters for the phenomenological model}

The phenomenological expression for the free energy of mixing of the bilayer, Eq.3.23, depends on several macroscopic parameters, namely: the bilayer thickness, $t_{h}$, the areal density of lipids, $\sigma_{\mathrm{m}}$, and the bending rigidity, $\kappa_{\mathrm{m}}$, per monolayer, the effective incompatibility or Flory-Huggins coefficient between unlike species, $\chi_{\text {eff, }}$, the intrinsic curvature-composition coupling, $\lambda$, and the average spontaneous curvature between the two lipid species, $\mathrm{C}_{0}$. As we have already seen in our study of area fluctuations in single component membranes (from where we obtained $\sigma_{\mathrm{m}}$ ), most of these parameters can be determined as independent measurements of different properties of the pure or mixed systems. Therefore, a complete interpretation of the results of our computer simulations, only requires the fitting of two of those parameters: $\lambda$ and $C_{0}$. The aim of this section is to describe how the rest of the parameters were determined.

\subsubsection{Susceptibility of composition fluctuations}

In this work, we study the interplay between the local curvature, $\mathrm{H}$, and the composition difference between the two monolayers, $\phi_{\text {eq, }}$ of the membrane. As seen when introducing the mean-field model, the difference in composition across the bilayer will be highly constrained by the overall composition of the system, $\psi$. Therefore, this study will require simulations at different $\psi$ values. 


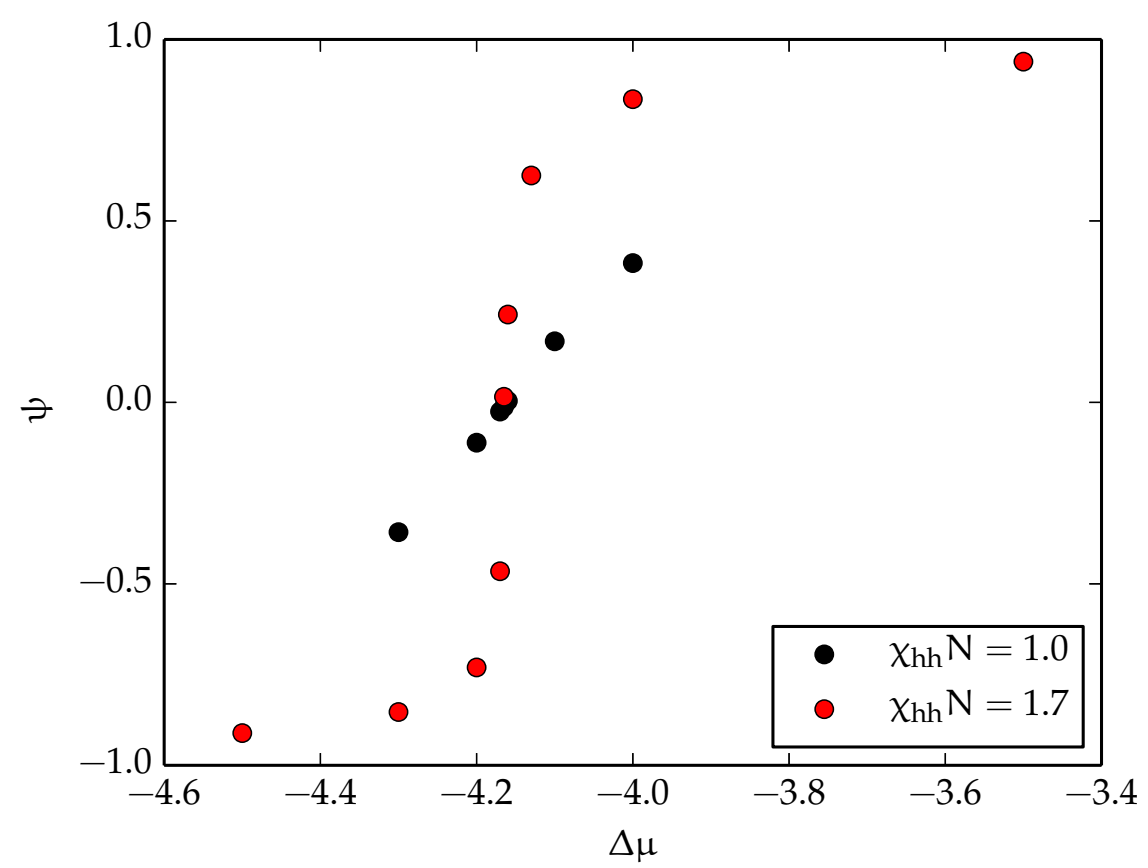

Figure 4.3: Average composition, $\psi$, as a function of the imposed difference in chemical potential between lipid species, $\Delta \mu$, for low and high Incompatibility between unlike head-group beads, $\chi_{h h} \mathrm{~N}$.

This can be achieved by several methods. One of them is setting the composition in both monolayers (beforehand) and then simulating the system in the canonical ensemble. A drawback of this scheme is that composition fluctuations are completely neglected. A second method allows the insertion and deletion of both lipids species, i.e., grand-canonical simulations. This approach has the additional advantage of incorporating fluctuations of both composition and total number of particles. Another option is to carry out the simulations in the semigrand canonical ensemble, which allows the mutation of one lipid species into the other, but the total number of particles in the system remains fixed. Here, the mutation rate is controlled by an imposed difference in chemical potential between species, $\Delta \mu$. Due to its easy implementation and high acceptance rate, we have adopted this last approach in our simulation studies. In addition to this, we have previously mentioned that the lateral sorting of lipids will be strongly influenced by $\chi_{\text {eff. }}$. In order to account for this effect, our simulations consider two regimes for the incompatibility between unlike head-group beads. These two regimes will be denoted as low and high incompatibility cases, and their corresponding incompatibilities will be given by $\chi_{h h} \mathrm{~N}=1.0$ and $\chi_{h h} \mathrm{~N}=1.7$.

The first task of our analysis for mixed systems, was to characterize the av- 


\section{CHAPTER 4. INTERPLAY BETWEEN CURVATURE AND COMPOSITION IN LIPID BILAYERS}

erage composition between the two membrane leaflets as a function of the imposed difference in chemical potential. This characterization is shown in Fig 4.3. There we can observe that, for low incompatibility, $\psi$ can be well described as a linear function of $\Delta \mu$. In contrast, for high incompatibility, the transition between the two single-component regimes occurs in a narrow interval around $\Delta \mu \sim-4.2$. This sharp transition denotes the proximity of the demixing critical point (characterized by $\chi_{\text {eff }}=2$ within the mean-field description Eq 3.13). Of course, our study will only make sense if the simulations for high incompatibility are within the mixed phase in the phase diagram. To verify this condition, $\Delta \mu$ was quasi-statically increased from its lowest value until we reached the upper rightmost, red point in Fig 4.3. From there, we followed the curve in the backward direction. While doing so, we didn't observed the appearance of any hysteretic behavior, that would be the signature of a first order phase transition. Furthermore, the probability distribution function of $\psi$ for all the simulations in the referred interval were unimodal, though the particular distribution for $\Delta \mu=-4.165$ exhibited small deviations from a Gaussian. This anticipates that, indeed, $\chi_{\text {eff }}$ is quite close to the mean-field critical value, $\chi_{c}=2$, but still smaller.

The chemical potential for the average composition of the mean-field model is given by the derivative of the bilayer free energy, Eq.3.23, with respect to $\psi$. This provides a direct method to evaluate $\chi_{\text {eff }}$ from the slope of the curves shown in Fig. 4.3 , since

$$
\left[\frac{\partial \psi}{\partial \Delta \mu}\right]^{-1}=\frac{1}{\sigma_{\mathrm{m}} A \mathrm{k}_{\mathrm{B}} \mathrm{T}}\left(\frac{\partial^{2} \mathrm{~F}}{\partial \psi}\right)=-\chi_{\mathrm{eff}}+\frac{2}{1-\psi^{2}} .
$$

Furthermore, the left hand side of this expression is nothing but the zero wavevector limit of the inverse susceptibility of composition fluctuations,

$$
\left[\frac{\partial \psi}{\partial \Delta \mu}\right] \equiv S_{0}=\lim _{\mathbf{q} \rightarrow 0} S(\mathbf{q})
$$

Within the random phase approximation and in the limit of small wave vectors, the structure factor can be approximated as $\underline{89 \mid 143-145}$

$$
S(\mathbf{q})=\frac{S_{0}}{1+\xi^{2} q^{2}}
$$

where $\xi$ is a constant related to the correlation length of concentration fluctuations. Therefore, measuring the fluctuation spectra of $\psi$ and extrapolating it towards $\mathrm{q} \rightarrow 0$, provides an alternative method for the evaluation of $\chi_{\text {eff }}$.

In Fig 4.4 we present the measurements for the susceptibility of composition fluctuations as evaluated from the slope of the data in Fig 4.3 (black dots) and 


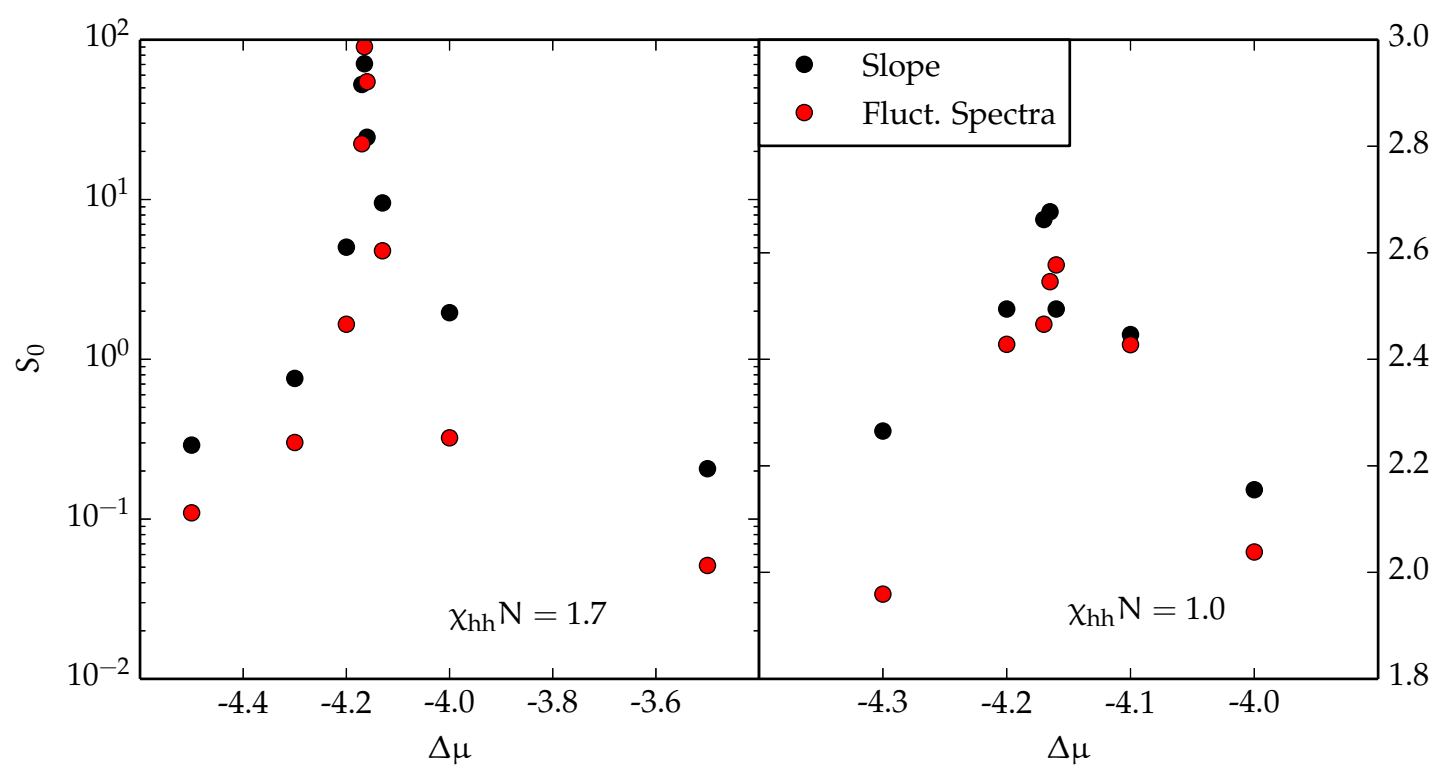

Figure 4.4: Susceptibility of composition fluctuations as evaluated from the slope of the $\psi$ vs $\Delta \mu$ curve (black) and from zero wave-vector extrapolation of $S(\mathbf{q})$ (red). The plot on the left (linear scale) shows the result from simulations with high incompatibility and the corresponding results for low incompatibility are shown on the right (logarithmic scale).

from the zero wave-vector extrapolation for the structure factor of composition fluctuations (red dots). First, we point out the good agreement between the two methods, although, in general, susceptibilities evaluated from the slope of the chemical potential are higher than those from the fluctuation spectra. Also, notice that for low incompatibility (right panel), the close proximity of data samples around $\Delta \mu \sim-4.165$ is prone to induce statistical errors in both methods (perhaps, this is seen more clearly from the black dots in Fig.4.3). However, appealing to linear response theory, $S_{0}$ was set as the slope of the linear regression for the $\psi$ vs $\Delta \mu$ data set.

On the other hand, for high incompatibility (left panel in Fig 4.4), we observe the expected enhancement of $S_{0}$ as the system approaches the critical composition, $\psi=0$ (i.e., $\Delta \mu \approx-4.165$ ). In accordance with equation $\mathrm{Eq}$.4.10, this results in an asymptotic approach of $\chi_{\text {eff }}$ towards the mean-field critical value, $\chi_{c}=2$, but always from below.

The incompatibilities resulting from this analysis are $\chi_{\text {eff }}=1.5 \pm 0.3$ and $\chi_{\text {eff }}=1.97 \pm 0.1$, 


\subsubsection{Density profile}

The next step in our analysis is to characterize the dependence of the bilayer thickness, $t_{h}$, on the leaflet composition. Similar to the discussion on the area per lipid, if the thickness difference between the two pure-component bilayers is small, we would expect a linear dependence on $\psi$, interpolating between the two composition extremes. However, if this condition is not met, the concomitant hydrophobic mismatch may result in higher-order contributions, modifying not only the mechanical properties of the bilayer, but also the lateral segregation of lipids.

An immediate question that arises when performing this analysis, is the definition of the bilayer thickness itself. Of course, there is not a unique way to define $t_{h}$ and a zoo of possible choices can be found in literature. A wide subset of definitions are based on the profile of mass distribution along the bilayer normal (the other large subset relies on the distribution of electrical charge across the bilayer). ${ }^{146}$ Among these, popular options include i) the peak-to-peak distance between the head-group distributions in apposing monolayers, ii) the peak-to-peak distance between backbones, i.e., the distance between the two hydrophobic-hydrophilic interfaces characterized by the aforementioned drop in the pressure profile (Fig 4.2) and iii) the full width at half maximum (FWHM) of the total hydrophobic contribution to the density profile. This last definition is commonly used in cases where the functionality of inclusions (such as integral membrane proteins) is strongly affected by hydrophobic mismatches. ${ }^{147}$ In this work, we adopted the first definition. The justification for this election is provided in the following paragraphs.

In Fig 4.5 we present the density profiles for systems with different composition and segregation conditions. From top to bottom, the first two plots correspond to pure, short and large head-group bilayers. Conversely, the last two plots stand for membranes with a vanishing average composition, i.e., $\psi \approx 0$, and for low and high incompatibility. In all the panels, distributions from the lower/upper monolayers $(z<0 / z>0)$ are traced with solid/broken lines. The individual contributions from hydrophilic beads in the short and large head-group lipids are colored in blue and red, respectively. The sum of these two hydrophilic contributions is shown in turquoise. From now on, we refer to this distribution as the total head-group distribution for the corresponding $\Delta \mu$ and $\chi_{\mathrm{hh}} \mathrm{N}$. Analogously, the contribution of the two lipid species to the hydrophobic profile of the individual monolayers is drawn in green and the total hydrophobic profile between the two leaflets is shown in gold.

Black solid line spanning trough all the panels, is located at the maximum of the total head-group distribution for $\Delta \mu=-2.0$ and $\chi_{h h} N=1.0$ (of the lower leaflet). Black broken lines in the lower panels indicate the location of 


\subsection{NON-FREE PARAMETERS FOR THE PHENOMENOLOGICAL MODEL}

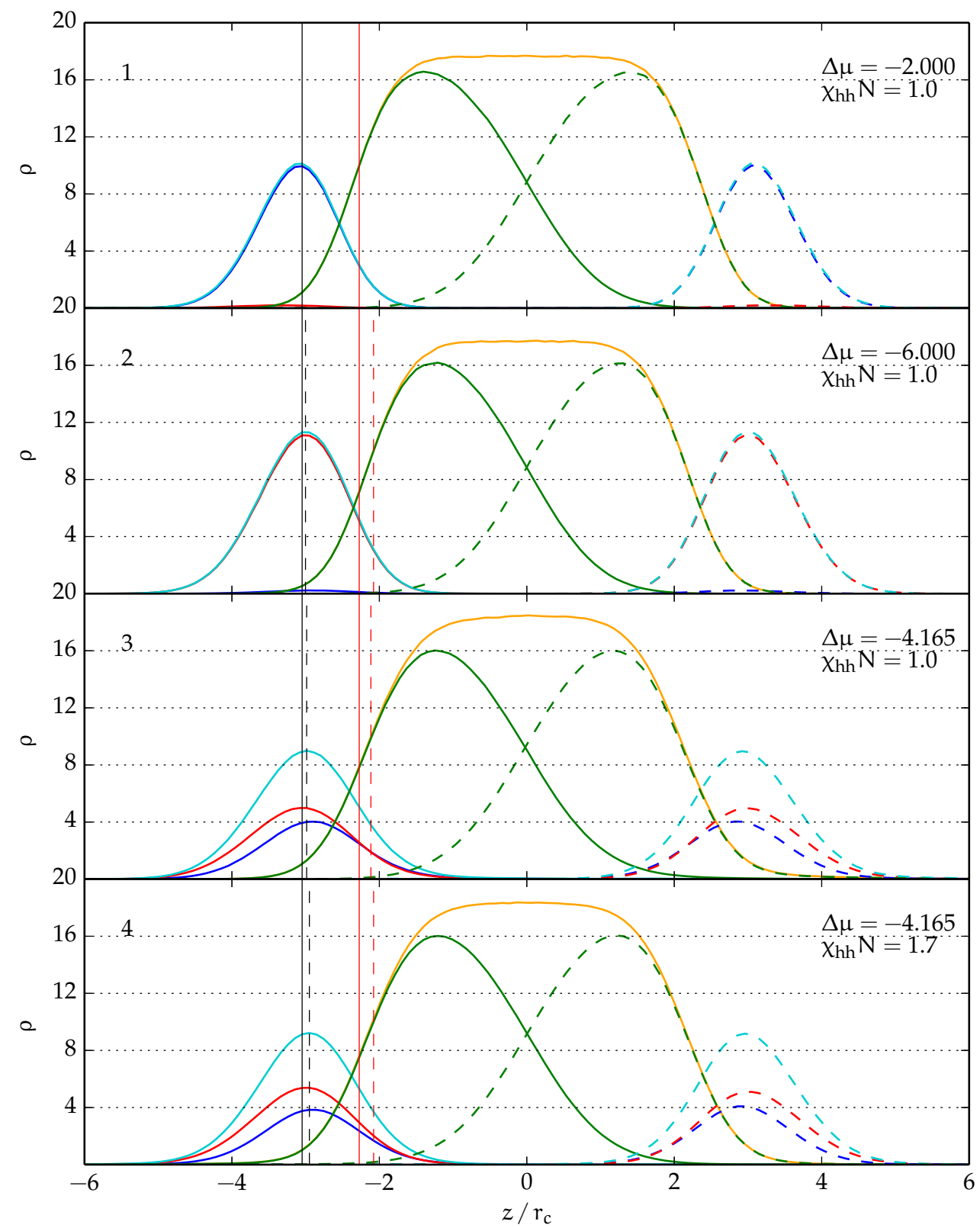

Figure 4.5: Density profile for different compositions and incompatibilities. From top to bottom (i.e., increasing label at the left of each panel), the first two plots correspond to pure B- and C-type bilayers. The last two plots correspond to systems with $\psi \approx 0$ and for low incompatibility and high incompatibility, respectively. 


\section{CHAPTER 4. INTERPLAY BETWEEN CURVATURE AND COMPOSITION IN LIPID BILAYERS}

the same maximum for each panel. In a similar manner, vertical, red, solid line is the locus of the left edge for the FWHM of the total hydrophobic profile, for $\Delta \mu=-2.0$ and $\chi_{h h} N=1.0$. Red broken lines in the other panels are the corresponding left boundaries for the given values of $\Delta \mu$ and $\chi_{h h} N$.

Let us begin by comparing the hydrophobic profiles of different systems. First, recall that tails in the B-type lipids (those whose pure bilayers are shown in the first panel), are one bead larger than tails in the C-type lipids. Also, the hydrophobic equation of state of both species was parametrized for the same compressibility and coexistence density, Eqs.2.6 and 2.8. These two facts would immediately account for the same height of the total hydrophobic plateau in the two, pure component systems (gold). They will also account for the shrinking of the FWHM in pure C-type and mixed bilayers. On the other hand, the small deviation between the low and high incompatibility cases may be attributed to the softness of the model and the degree of segregation in the system: in well mixed bilayers, the presence of unlike neighbors will induce stretching or compression of the tails (depending on its length) to avoid an hydrophobic density mismatch. However, the net cost of this homogenization would be a shift of the overall hydrophobic density, in comparison to that of pure component bilayers. Of course, the preference of one kind of deformation over the other would result from the subtle balance between the chain stiffness and the compressibility of the hydrophobic interior. In the mixed belayer, there is an additional repulsion in the head-group region. This leads to a slightly larger area per head group and, at fixed tail density, to a thinning of the bilayer. This is in agreement with the results shown in panels 3 and 4, which indicate that, indeed, compressions are preferred over elongations, as it is reflected by the slight increase of the hydrophobic plateau level. As segregation increases and, in particular, when the system is driven towards the vicinity of the demixing critical point (as is the case for $\Delta \mu=-4.165$ and $\chi_{\mathrm{hh}} \mathrm{N}=1.7$ ), composition inhomogeneities show up all over the membrane. At the interior of these inhomogeneities, lipid stretching will resemble that of pure component systems and only the lipids at the domain edge will experience an environment similar to that of well-mixed bilayers. Therefore, it is expected that these highly-segregated systems exhibit hydrophobic profiles in between those for pure component and well-mixed bilayers.

A final remark on the hydrophobic profiles is, as already mentioned, the lack of a dip at the interface between the two monolayers. This dip has been observed in simulations with atomistic and systematically coarse-grained models $\frac{148 \mid 149}{}$ and its origin has been attributed to excluded volume interactions, which effectively prevent the interdigitation of the apposing monolayers. Our lipid parametrization, however, seems to make the lipid ends too loose, there- 
fore missing the development of this dip.

Now we turn to the description of the head-group distributions. Given the fact that C-type head-groups are one bead larger than the B-type ones (as reflected by the higher peak value in the 2nd panel), a simple geometrical argument would predict a $\sim 4 \%$ shorter peak-to-peak distance in the former case. If that were the case, such difference would be as large as the one shown in panel 4. However, a concentration increase, together with good solvent conditions, will enhance the swelling of the largest head-group species, hence resulting in a bigger peak-to-peak distance than the one expected from the geometrical argument. In contrast, head-groups swelling in mixed system will be highly influenced by the balance between beads solubility, favoring the dilution into the vacuum-solvent, and the incompatibility between unlike species, promoting the formation of clusters. The outcome of our simulations with mixed systems (panels 3 and 4) shows that large head-groups will prefer to remain elongated, whereas the short ones will slightly contract, in order to maximize the contact with alike beads. This effect can be clearly seen by noticing that the maximum of the large head-group distributions (in red) is farther away from the bilayer midplane than the corresponding maximum for the short head-group (blue). This situation is contrary to what would be expected from the geometrical argument. The net result of this elongation of large head-groups and contraction of short ones, will be a total head-group distribution (shown in turquoise) closer to the bilayer midplane than in pure component systems. For most of our simulations, however, this effect is smaller than what is expected from geometrical arguments. Therefore, in later analysis of the interplay between curvature and composition, we have set the bilayer thickness as the average of all the hydrophilic peak-to-peak distances, namely, $t_{h}=6.05 r_{c}$. Furthermore, since typical thickness values for biological, fluid membranes are in the range of 5-7 $\mathrm{nm}$, we have identified the length unit of our simulations as $r_{\mathrm{c}} \approx 1 \mathrm{~nm}$.

\subsubsection{Power spectra of height fluctuations}

As it was shown in our discussion of the elastic membrane models in Sec 3.1.1. Eq 3.8, bilayer bending rigidity can be evaluated from the small wave-vector limit of the power spectra of height fluctuations, $\left\langle\left|h_{\mathbf{q}}\right|^{2}\right\rangle$. To evaluate the fluctuation spectra from our simulations, every membrane configuration was mapped into a two-dimensional grid of $16 \times 16$ parallelepipeds, each spanning the entire simulation box along the membrane normal. Then, the local membrane height, $h_{i j}$, was calculated as the center of mass, along the axial direction, of all the beads within the current parallelepiped. The fluctuation spectra was obtained from the two-dimensional, discrete Fourier transform of the local height, $h_{\mathbf{q}}$, 
and the evaluation of $\left|h_{\mathbf{q}}\right|^{2}$.

$$
\left\langle\left|h_{\mathbf{q}}\right|^{2}\right\rangle=\frac{k_{B} T}{L^{2}\left[k q^{4}+\gamma q^{2}\right]}
$$

In Fig 4.6 we present the power spectra for different incompatibilities and chemical potentials. From the results shown in the left panel, we conclude that, for well-mixed systems (i.e., at low incompatibility), membrane elastic properties are independent of composition. This result is consistent with the previous analysis on the area per lipid and the bilayer thickness, where we found that the overall length and cross-sectional area of both lipid species are practically the same. Having these two quantities a large influence on the rigidity of the bilayer, $150 \mid 151$ the independence on composition is not surprising. In contrast, for high incompatibility, one would expect that the tendency to form domains of lipids with the same spontaneous curvature, will have a noticeable influence on membrane shape fluctuations. However, results from the simulations with high segregation (shown in the right panel) do not exhibit systematic differences neither between systems with different compositions nor when compared to the low incompatibility case. In the end, bending rigidities obtained after averaging over different compositions resulted in $\kappa=20.48 \pm 0.5$, for low incompatibility, and $k=21.98 \pm 2.7$, for high incompatibility. Since these results are identical within the error bar, the final bilayer bending rigidity is set as their average,

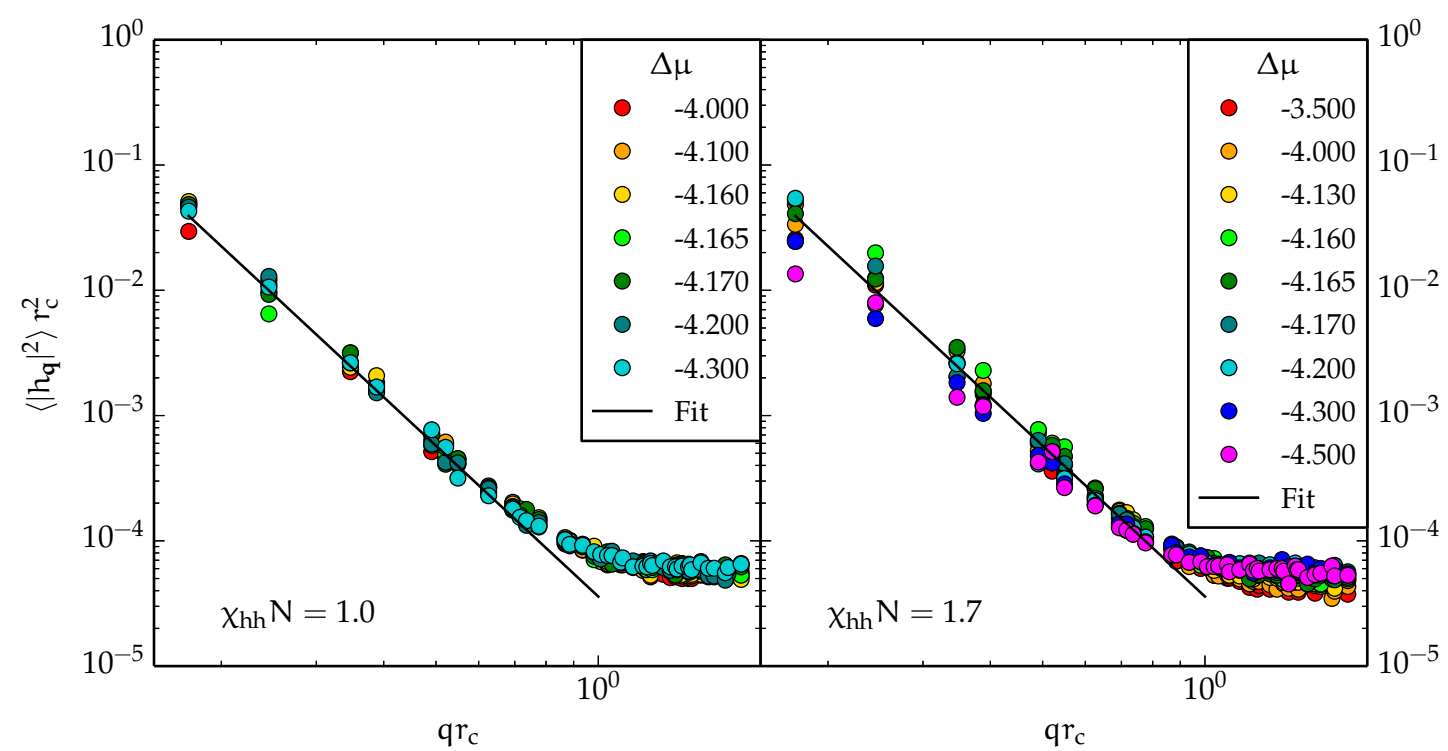

Figure 4.6: Spectra of height fluctuations for different chemical potentials. Results for low/high incompatibilities are shown in the left/right panel. 
$\kappa=21.2 \pm 2.7$.

Finally, we want to point out that results obtained from this analysis are in good agreement (although slightly smaller) with the rough estimation from the susceptibility of area fluctuations, $\mathrm{Eq} 4.6$, for the pure component systems. More importantly, these values are well within the reported values for biologically relevant, membrane-forming lipids. $\frac{1521153}{}$

\subsubsection{Summary}

In this section, we have provided a detailed description of the methods used to independently determine most of the parameters in the mean-field model for the free energy of mixing of bilayers composed of lipids with different spontaneous curvatures in Eq 3.23. We have seen that, for the particular parametrization of the coarse-grained model used in this work, changing the relative length of the head-group block by one bead, while keeping the total number of beads per chain fixed, results in lipid species whose overall length and cross-sectional area are very similar. An immediate consequence of this is that, under wellmixing conditions, the elastic properties of both pure and mixed bilayers will be similar too. However, as we pointed out when analyzing the pressure profile of pure bilayers, the similarity of overall lateral extensions does not imply that both lipid species should have the same intrinsic curvature. We have envisioned that this curvature difference would influence the elastic properties of mixed bilayers under strong segregation conditions. Nevertheless, the power spectra of shape fluctuations, do not exhibit any systematic dependence on composition. This apparent contradiction, however, can be rationalized in terms of the model refinement introduced in Sec.3.3. There, we illustrated how the corrections due to a curvature-dependent bending rigidity, would not have a significant effect on the lipid sorting of planar membranes undergoing thermally-induced shape fluctuations (region $t_{h} \mathrm{H} \sim 0$ in Fig.3.7). Since such curvature dependence can be translated into a composition-dependence, via the curvature-composition coupling, the previous reasoning can be used to explain why composition dependent contributions to the bending rigidity are negligible when compared to the bare contribution, at least within the range of curvatures accessible to undulating planar bilayers.

The final values of the parameters to be used in our analysis of the curvaturecomposition coupling are enlisted in $\mathrm{Tab} 4.3$ 
CHAPTER 4. INTERPLAY BETWEEN CURVATURE AND COMPOSITION IN LIPID BILAYERS

Table 4.3: Summary of bilayer properties used in the curvature-composition coupling analysis.

\begin{tabular}{lclr}
\hline \hline Quantity & Symbol & Unit & Value \\
\hline Bilayer thickness & $t_{h}$ & $n m$ & 6.0 \\
Average area per lipid & $\langle a\rangle$ & $\mathrm{nm}^{2}$ & 0.56 \\
Incompatibility for high segregation & $\chi_{\text {eff }}$ & $\mathrm{k}_{\mathrm{B}} \mathrm{T}$ & 1.97 \\
Incompatibility for low segregation & $\chi_{\text {eff }}$ & $\mathrm{k}_{\mathrm{B}} \mathrm{T}$ & 1.50 \\
Bilayer bending rigidity & $\mathrm{K}$ & $\mathrm{k}_{\mathrm{B}} \mathrm{T}$ & 21.2 \\
\hline \hline
\end{tabular}

\subsection{Effective curvature-composition coupling}

Once the incompatibility between lipid species, as well as the thickness, areal density and bending rigidity of the bilayer have been identified, there are only two parameters left $\left(\lambda\right.$ and $\left.C_{0}\right)$, to completely characterize the thermodynamic state of the membrane, according to the mean-field model, Eq.3.23. When studying the properties of this model, we found that, under specific segregation conditions, the difference in composition between the two leaflets, $\phi$, is linearly related to the local curvature of the bilayer midplane, $\mathrm{H}$, via the effective curvature-composition coupling, $\Lambda_{\text {eff, }}$

$$
\phi=\Lambda_{\mathrm{eff}} \mathrm{H}
$$

As discussed, beside depending on the elastic and geometrical properties of the membrane (the latter ones including $\lambda$ and $C_{0}$ ), the effective coupling also depends on the average composition between the two leaflets. Furthermore, we argued that the specific segregation conditions where this linear relationship holds, are usually met within the range of curvatures accessible to planar membranes in the presence of thermally-induced shape fluctuations. Therefore, measuring $\phi$ and $\mathrm{H}$ in our simulations with planar bilayers, and afterwards fitting the mean-field expression for the effective coupling, Eq 3.25 , provides a simple way to determine the intrinsic curvature-composition coupling, $\lambda$, and $\mathrm{C}_{0}$, parameters directly related to the spontaneous curvature of the individual lipid species via Eq 3.15 .

\subsubsection{Joint probability distribution for $\phi$ and $H$}

Following a mapping procedure similar to the one used to evaluate the power spectra of shape fluctuations, the local composition difference between the two membrane leaflets, as well as the height of the bilayer midplane were mapped 


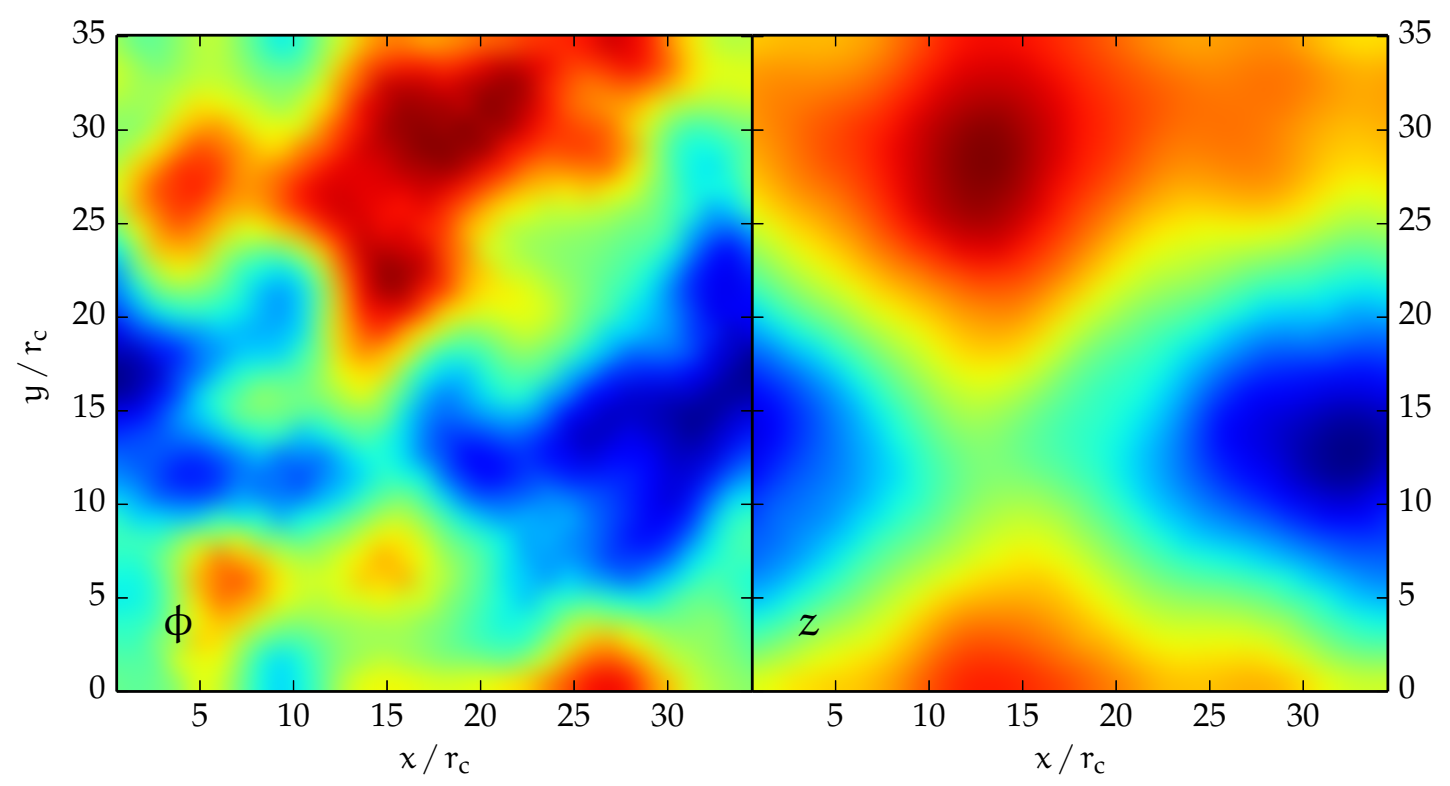

Figure 4.7: Interpolated grid mappings of the composition difference between the two apposing monolayers, $\phi$, and the height of the bilayer midplane.

into a square grid, parallel to the plane of the membrane. An interpolated image of these mappings is shown in Fig 4.7. The particular configuration used to generate them, belongs to simulations in the close vicinity of the demixing critical point. That means, they are characterized by a high incompatibility $\left(\chi_{\mathrm{hh}} \mathrm{N}=1.7\right)$ and $\Delta \mu=-4.165$ (where $\psi \sim 0$ ). According to our phenomenological description of the sorting mechanism, Sec,3.2, these conditions will enhance the strength of the effective curvature-composition coupling. That is clearly seen in the high degree of correlation between both mappings.

To evaluate the local curvature, every point on the height mapping was fitted (within a local sub-mesh of $4 \times 4$ grid points) by a quadric function of the form

$$
g(x, y)=p_{1} x^{2}+p_{2} y^{2}+p_{3} x y+p_{4} x+p_{5} y+p_{6} .
$$

In this Monge-like parametrization, the local mean curvature is given by 20

$$
\mathrm{H}=\frac{\left(1+g_{y}^{2}\right) g_{x x}-2 g_{x} g_{y} g_{x y}+\left(1+g_{x}^{2}\right) g_{y y}}{2\left(1+g_{x}^{2}+g_{y}^{2}\right)^{3 / 2}},
$$

where subindeces stand for the partial derivatives of $g(x, y)$, with respect to the corresponding variable. Within the limit of small curvatures, this expression further simplifies to

$$
\mathrm{H}=\frac{g_{x x}+g_{y y}}{2}=p_{1}+p_{2} .
$$




\section{CHAPTER 4. INTERPLAY BETWEEN CURVATURE AND COMPOSITION IN LIPID BILAYERS}

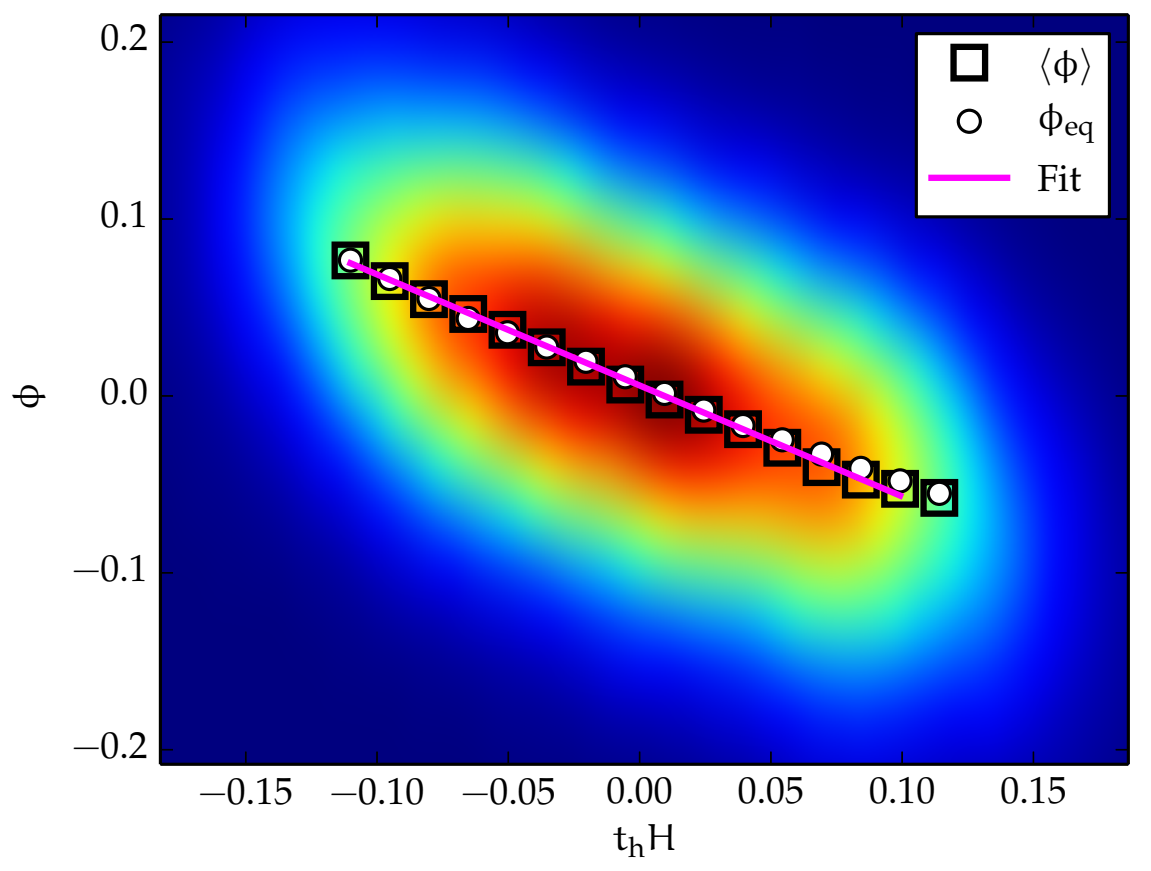

Figure 4.8: Joint probability distribution for the local curvature, $\mathrm{H}$, and the composition difference between monolayers, $\phi$. It was evaluated from the simulation run with $\chi_{\mathrm{hh}} \mathrm{N}-1.7$ and $\Delta \mu=-4.130$. Average, $\langle\phi\rangle$, and most probable values, $\phi_{\text {eq, }}$ of particular distributions at fixed curvature are shown in squares and circles, respectively. The the solid line is the linear fit for the most probable data set.

Once curvature mappings were computed, the joint probability distribution for the local curvature and composition, $\mathrm{P}(\mathrm{H}, \phi)$, was evaluated from the correlation of all the corresponding mappings, for a given incompatibility and difference in chemical potential (i.e., average composition between leaflets). For each of these distributions, we computed the average, $\langle\phi\rangle$, and the most probable, $\phi_{\text {eq }}$, composition differences at fixed curvature (shown in squares and circles, respectively). Away from the demixing critical point, $\phi$ is Gaussian-distributed and these two quantities coincide. However, as criticality is approached, the emergence of correlations on length scales comparable to the system size, undermines the validity of the central limit theorem and deviations from the Gaussian distribution arise. ${ }^{154}$ As already pointed out when studying the susceptibility of composition fluctuations, in our simulations such small deviations were only observed for high incompatibility and $\Delta \mu=-4.165$.

Finally, the effective curvature-composition coupling, for each incompatibility and average order parameter, $\psi$, was evaluated as the slope of the linear fit 


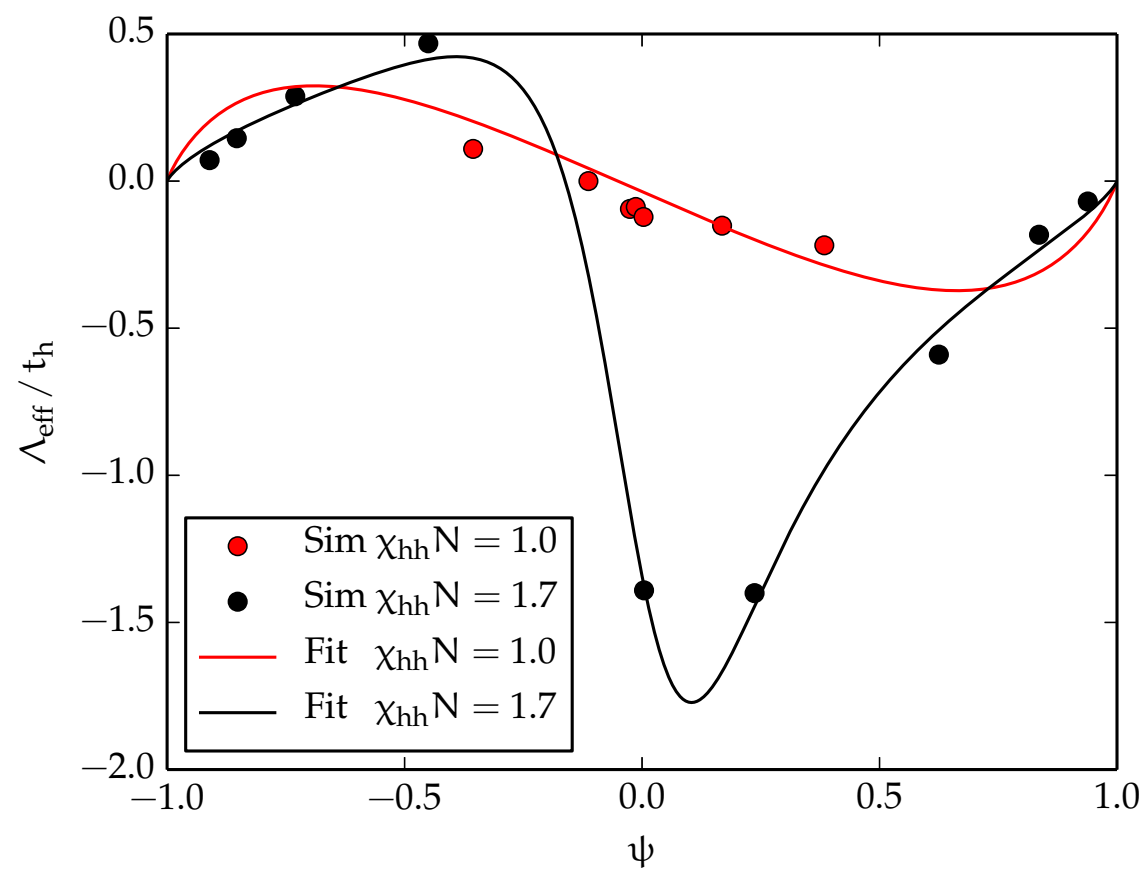

Figure 4.9: Effective curvature-composition coupling as a function of the average order parameter, $\psi$. Data sets obtained from the slope of the $\phi_{\mathrm{eq}} \mathrm{vs} \mathrm{H}$ curve for high and low incompatibility, are shown in red and black circles, respectively. The global fit of the phenomenological expression for the effective coupling, Eq 3.25 , is given by the corresponding continuous lines.

of the $\phi_{\text {eq }}$ vs $H$ curve. Then, our mean-field expression for $\Lambda_{\text {eff }}$ as function of $\psi$, was globally fitted for the low and high incompatibility data sets. The result of these measurements and fits are shown in Fig.4.9.

A first remarkable feature to be pointed out from these results is the good agreement between simulations and the phenomenological theory (notice that the two continuous lines belong to a single fit). Also, in agreement with our previous analysis of the mean-field model, we verify that the effective curvaturecomposition coupling is enhanced in the close proximity of the demixing critical point. In that case, the strong segregation between lipids undermines the ability of monolayers to accommodate lipid flip-flops, since new insertions would be preferably located near alike species, therefore reaching saturation conditions at smaller curvatures than those in the mixed case. Perhaps, this effect is better visualized in the snapshots of particular configurations from low (left) and high (right) incompatibility simulations, shown in Fig 4.10. Although in both cases the height of the bilayer is modulated by the effect of thermal fluctua- 


\section{CHAPTER 4. INTERPLAY BETWEEN CURVATURE AND COMPOSITION IN LIPID BILAYERS}

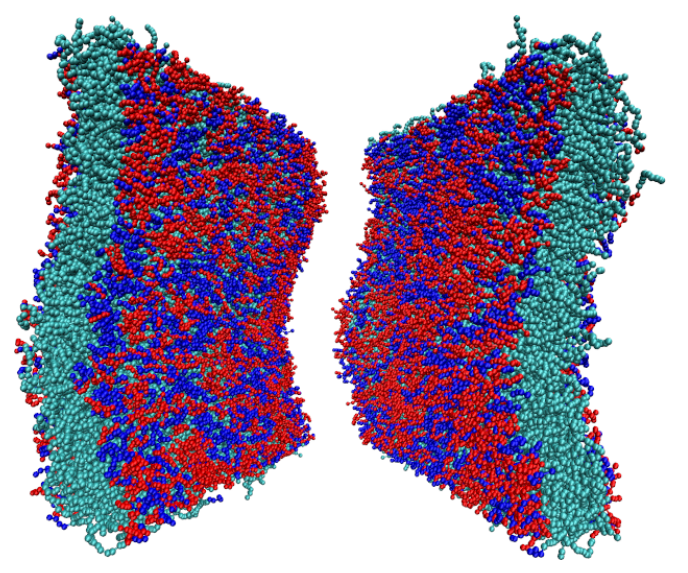

(a) Low incompatibility
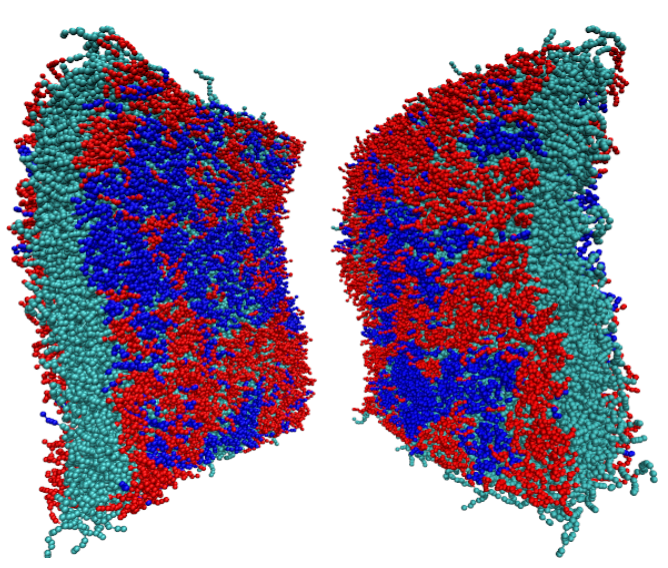

(b) High incompatibility

Figure 4.10: Comparison of the lateral sorting of lipids in the upper and lower leaflets of the bilayer, from simulations with low (left) and high (right) incompatibility.

tions, for low incompatibility the lateral segregation of lipids and the curvature of the bilayer are uncorrelated. Conversely, for high incompatibility, short headgroup lipids in the upper and lower leaflets (in blue), tend to cluster into regions where the bilayer is locally curved towards its normal (concave deformations), whereas large head-group species (in red) prefer to accommodate within regions curved in the opposite direction, where the accessible area per lipid is increased.

Results in Fig 4.9 also reproduce the expected behavior for single-component systems (those with $\psi= \pm 1$ ) where the effective coupling vanishes, since the composition difference remains zero, regardless of the curvature of the bilayer is. Furthermore, our simulations exhibit the predicted lack of symmetry with respect to the $\Lambda_{\text {eff }}$ axis, which reflects the natural tendency of the system to favor the proper sorting of lipids based exclusively on their geometry.

Finally, the fitted values for the intrinsic coupling and the average curvature of both lipid species are $\lambda=2.01 \mathrm{~nm}$ and $C_{0}=-0.27 \mathrm{~nm}^{-1}$. The corresponding spontaneous curvatures for the individual lipid species obtained from these values via Eq.3.15, are $C_{+1}=0.072 \mathrm{~nm}^{-1}$ and $C_{-1}=-0.61 \mathrm{~nm}^{-1}$. First of all, these results are consistent with those presented in Tab.4.1, where short head-group lipids $\left(C_{+1}=0.072 \mathrm{~nm}^{-1}\right)$ are reported to self-assemble into planar bilayers, whereas large head-group ones are close to the phase boundary between planar bilayers and cylindrical micelles. Also, these spontaneous curvatures are in good agreement with experimental results from biologically relevant membrane-forming lipids. $155-157$ 


\subsection{Cylindrical bilayers}

Results presented in the previous section validate the hypothesis that, far from saturation, the composition differences for a given order parameter (recall that $\left.\phi_{\text {eq }} \in[-1+|\psi|, 1-\psi]\right)$, is linearly related to the local curvature via the effective curvature-composition coupling. A natural question, however, is what happens in systems where such conditions are not met. Examples of those occur in membrane-pulling experiments, where cylindrical membrane tubes, whose midplane radius of curvature is comparable to the bilayer thickness, are pulled out (via micropipette aspiration) from giant unilamellar vesicles. We argue that the lateral sorting of lipids in such systems will be duly described by the implicit relation between $\phi_{\text {eq }}$ and $\mathrm{H}, \mathrm{Eq} \cdot 3.23$. To verify this hypothesis, we have done semi-grand canonical simulations with cylindrical membranes of different radii (as those shown in Fig4.11a), for the two incompatibility regimes and for a difference in chemical potential $\Delta \mu=-4.160$. We have chosen these two cases since they should exhibit a good contrast between the saturation behavior for low and high incompatibility, while avoiding the finite size effects emerging in the close proximity of the demixing critical point. The corresponding average compositions for low and high incompatibility are $\psi=0.004$ and $\psi=0.242$.

Of course, the self-assembly of the lipid species into cylindrical bilayers is not a spontaneous process and their simulation required the pre-assembly of such cylinders. The relaxation from these metastable states towards the equilibrium planar configurations, however, is an enormously slow process. The reason for this is the high barrier in the energy landscape, due to the exposure of hydrophobic interior to the vacuum-solvent.

In order to equilibrate the number of lipids on each monolayer (which should be proportional to their surface area), we opened two pores perpendicular to the cylinder axis. These pores allowed the exchange of lipids between the inner and the outer monolayers. The blank spot in the axial cut of Fig $4.11 \mathrm{~b}$ shows one of these pores. These pores were created by applying an external hydrophobic potential of the form,

$$
\mathcal{U}_{\text {pore }}=-k_{\mathrm{p}}\left(\left|\mathbf{r}-\mathbf{r}_{\mathrm{p}}\right|-\mathbf{R}_{\mathrm{p}}\right)^{2} \text {, }
$$

where $\mathbf{r}_{p}$ denotes the center of the pore, $k_{p}$ is the force constant of the potential and $\mathbf{R}_{\mathrm{p}}$ is a cutoff distance which ultimately defines the radius of the pore. Acting exclusively on tail beads, this potential creates a depletion region within the hydrophobic interior, therefore triggering the redistribution of the hydrophilic head-groups around the pore to shield the bilayer interior. In the end, this redistribution creates an hydrophilic channel through which lipids can diffuse from one monolayer to the other. 


\section{CHAPTER 4. INTERPLAY BETWEEN CURVATURE AND COMPOSITION IN LIPID BILAYERS}

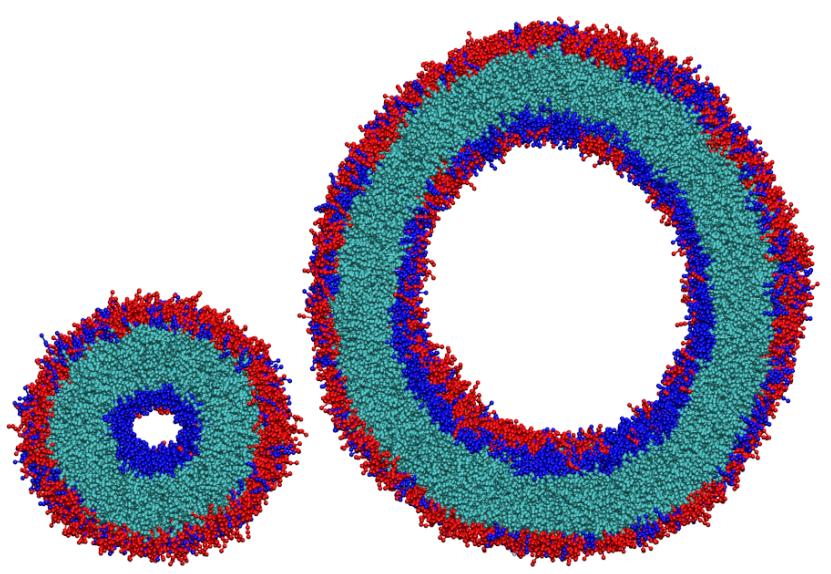

(a) Cylinders of different radius

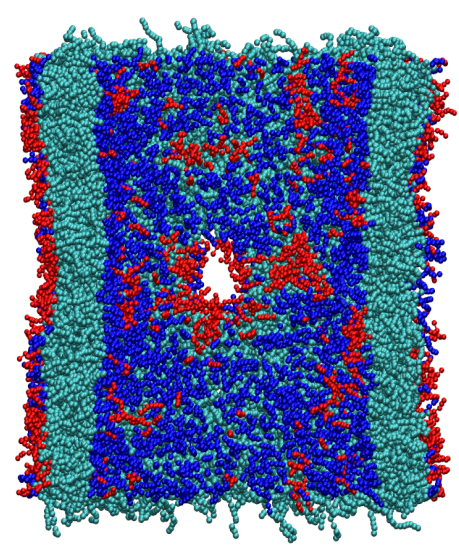

(b) Cylinder with pore

Figure 4.11: Comparative of the different cylinder radii and axial cut showing the pore used to equilibrate leaflets composition.

Once the surface density in both monolayers was equilibrated, pores were closed and the recording of statistical properties began. In contrast to the local analysis done for planar bilayers, in this case we computed the average radius and composition of the entire, individual monolayers. The reason for this is that instantaneous configurations exhibited only minor deviations from the cylindrical geometry (as can be clearly seen in the snapshots). Therefore, the bilayer radius (computed as the average value between the two monolayers) provided an accurate measurement of system curvature.

To compare the results from different tube simulations and the predictions of phenomenological model, we took the set of parameters obtained in the analysis of planar bilayers (reported in Tab 4.3, together with the values of $\lambda$ and $\mathrm{C}_{0}$ obtained from the fit of the effective coupling), and plugged them into the implicit relation for $\phi_{\text {eq }}$ and $H, E q .3 .24$. The solutions of this relation for the two incompatibility cases (red and black continuous lines), together with the corresponding data from planar (circles) and cylindrical (squares) configurations, are shown in Fig 4.12. The first remarkable feature is the nice agreement between simulations in both curvature regimes and the model predictions for both incompatibilities. Here we want to stress that the parameters $\lambda$ and $C_{0}$ used to generate the solid lines, were obtained from a global fit of the effective coupling datasets for low and high incompatibility, shown in Fig 4.9

Another remarkable feature is the anticipated contrast between both incompatibility cases, concerning the transition towards the saturation regime: at low incompatibility, the well-mixing conditions shift the saturation curvature beyond the geometrical limits of the system. This turns the effective curvature- 


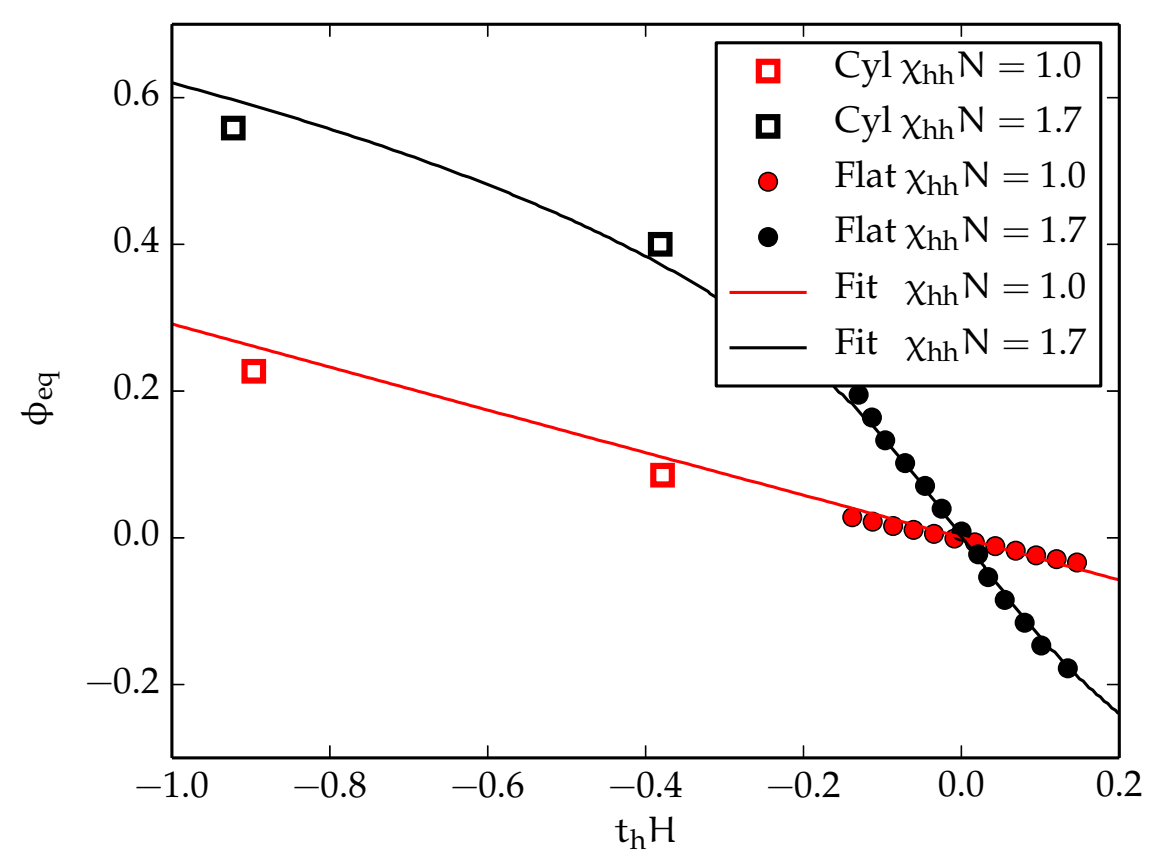

Figure 4.12: Comparison between the simulated equilibrium composition differences and curvatures of planar (circles) and cylindrical bilayers (squares) and the solution of the implicit, mean-field relation Eq 3.24 (continuous lines). Results for low and high incompatibility are shown in red and black, respectively. All plots correspond to system with $\Delta \mu=-4.160$.

composition coupling into the only relevant quantity to describe the interplay between curvature and the lateral sorting of lipids. Conversely, the entropy loss associated with a high degree of segregation, favors the emergence of a finite curvature beyond which the composition asymmetry across the bilayer is not dominated by the addition of alike lipids to already formed composition domains, but by local lipid rearrangements within these domains, thus undermining the effectiveness of the sorting mechanism. 
CHAPTER 4. INTERPLAY BETWEEN CURVATURE AND COMPOSITION IN LIPID BILAYERS 


\section{Chapter 5}

\section{Spontaneous curvature from inverted lipid structures}

\subsection{Self-assembly of inverted structures}

So far, the discussion has focused on the study of bilayer structures with different geometries and under qualitatively different segregation conditions. However, in Chapter 1 we saw that, beside bilayers, biologically relevant lipids can self-assemble into inverted structures displaying liquid-crystalline order. $158[159$ An example of is the inverted hexagonal phase (schematics of this and other inverted phases are shown in Fig.5.1). These phases consist of monolayers rolled up into long cylinders with the lipid head-groups pointing towards the cylinder axis and the hydrophobic tails located at the outer shell of the monolayer. This particular lipid orientation prevents the occurrence of inverted phases at low concentrations, because the hydrophobic part is left unshielded from the solvent. Furthermore, at high concentrations, the hydrophobic effect will result in densely packed structures where the close contact between neighboring, inverted monolayers tries to be maximized.

In Chapter 1, we also discussed that, in an ideal scenario, lipid self-assembly will result in tensionless configurations where the radius of the cylinder and the intrinsic spontaneous curvature of the individual monolayers coincide. $\frac{76] 160}{}$ One often assumes that the spontaneous curvature of such tensionless systems is an additive function of the curvature of the individual lipids or, in other words, the monolayer spontaneous curvature is a linear function of its composition, as expressed by Eq.3.14. This hypothesis has been validated by the results from simulations with lipids bilayers presented in Chapter 4 . These ideas provide an immediate approach to measure the spontaneous curvature of lipid species, other than the ones comprising the single-component inverted 


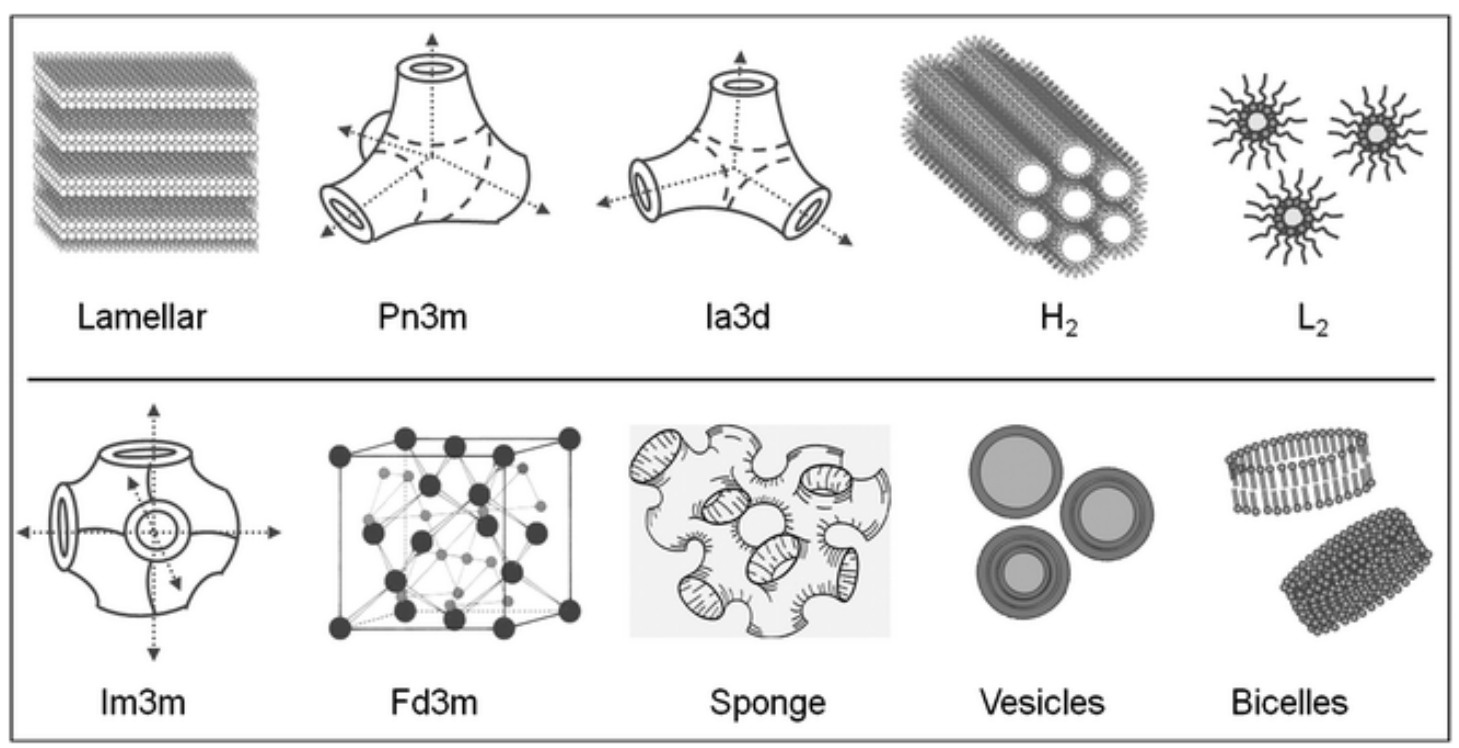

Figure 5.1: Schematic representation of the different lipid inverted phases. Monoolein shows lamellar, bicontinuous cubic-Pn3m and Ia3d phases, hexagonal (H2) and fluid isotropic (L2) phases in the presence of water (upper row). Additional structures (bottom row) like bicontinuous cubic phase-Im3m, micellar cubic-Fd3m, sponge phase, vesicles and bicelles are also formed by monoolein but in presence of additives, for example, other lipid(s), detergent or salt etc. in water. Image reproduced from Kulkarni et al. [159].

phase. Starting from a tensionless and single-component structure with spontaneous curvature $C_{\text {host }}$, the addition of lipids with a different intrinsic curvature, $\mathrm{C}_{\text {guest }}$, will result in a mixed inverted structure with curvature

$$
\mathrm{C}_{\text {mix }}=\left(1-\phi_{\text {guest }}\right) \mathrm{C}_{\text {host }}+\phi_{\text {guest }} \mathrm{C}_{\text {guest }} .
$$

Then, the spontaneous curvature of the guest species can be determined by measuring the curvature of the mixed inverted cylinders for different lipid concentrations. Usually, the radius of the resulting cylinder is determined from $x$-ray measurements of the lattice dimensions of the inverted structure. ${ }^{126160}$

Well-mixing conditions between the two species is a mandatory requirement to characterize the intrinsic lipid curvatures via Eq5.1. If these conditions are not met, the guest lipids will segregate into specific regions of the monolayer, therefore destroying the cylindric symmetry of the system, as it is shown in Fig 5.2 for different concentrations. Here, it is important to stress that the presence of two monolayers with opposite curvatures prevents this situation to occur on spherical vesicles or membranes tubes. This is because, in these systems, lipids can flip-flop or redistribute in a coordinated fashion between the two 


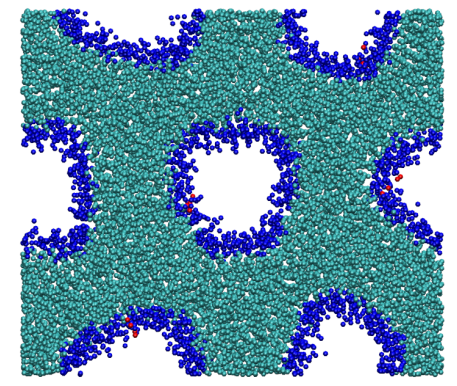

(a) $\phi_{\text {guest }}=0.004$

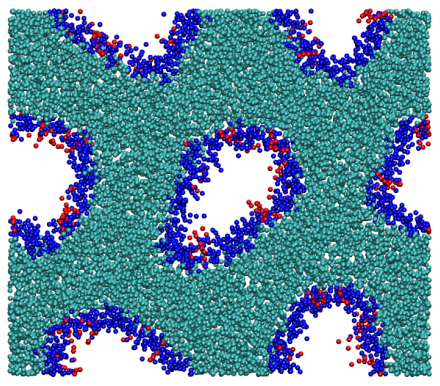

(b) $\phi_{\text {guest }}=0.1$

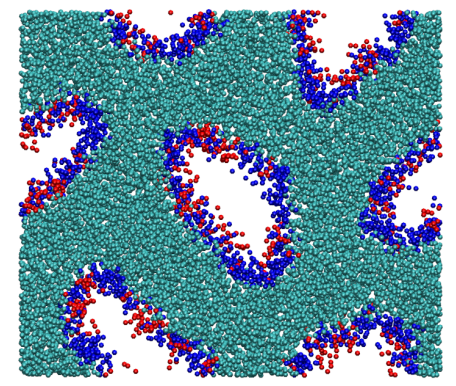

(c) $\phi_{\text {guest }}=0.2$

Figure 5.2: Anisotropic deformations of the inverted hexagonal phase due to the high incompatibility between host and guest lipid species. The lipid architecture of the host species comprises 3 head-group beads (shown in blue) and 13 tail beads (shown in green). Analogously, the guest species comprises 4 headgroup beads (shown in red) and 12 tail beads (also shown in green). The lateral dimensions of the simulation box for the 3 snapshots are $L_{x}=23.2, L_{y}=20.09$ and $\mathrm{L}_{z}=5.4$, where the $\mathrm{L}_{x}$ is parallel to the sheet short side and $\mathrm{L}_{z}$ points out of the page. The incompatibility between unlike head-group species is the same as the low incompatibility case in the simulations with bilayer structures, $\chi_{h h} \mathrm{~N}=1.0$.

leaflets, in order to match their curvatures.

Another caveat of this method is that, as discussed in Chapter 1, the linear additivity of the individual lipid curvatures to the spontaneous curvature of the resulting aggregate is only possible when the lipids geometry is left unchanged in the mixed assemble. However, we have seen that, in order to prevent the occurrence of voids inside the hydrophobic region, lipids may need to undergo isochoric deformations, which ultimately modify their intrinsic curva-

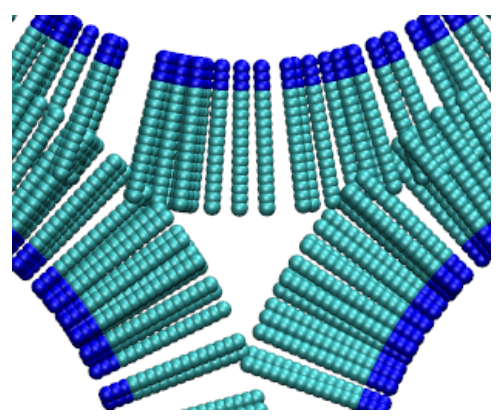

Figure 5.3: Schematic representation of the voids between tubes in the inverted hexagonal phase. 
ture. On self-assembled bilayer structures (i.e., planar membranes, bilayer tubes or vesicles) the presence of voids is greatly hindered because of the curvature complementarity of the apposing monolayers. However, these voids would be particularly prone to occur at the intersection between three of more inverted monolayers, as schematically shown in Fig.5.3 for the inverted hexagonal case. Therefore, the geometry of lipids at these locations may be considerably different from that of lipids sitting at the locations where the distance between inverted monolayer is minimal. This anisotropic conditions will influence the sorting of both host and guest lipids species and their net effect will ultimately be reflected on the global curvature of the mixed system, $C_{\text {mix }}$. These effects, however, are completely neglected by Eq.5.1.

\subsection{Prevention of void-induced stretching}

A way to prevent stretching effects at the intersection between monolayers is to suspend the inverted-cone shaped lipids into an incompressible, hydrophobic solution. Under such conditions, self-assembled monolayers will be less urged to maximize the contact with neighboring aggregates and, instead, will be effectively surrounded by the hydrophobic solvent, therefore resulting in the formation of inverted worm-like micelles. 161/162

The Helmholtz free energy of a single monolayer immersed into an incompressible hydrophobic solvent of lipid tails will be given by

$$
F(D, L)=\frac{\kappa_{m}}{2} A\left(R^{-1}-C_{\phi}\right)^{2}+\frac{\kappa_{A}}{2} \frac{\left(A-A_{\phi}\right)^{2}}{A_{\phi}},
$$

where $\mathrm{D}$ and $\mathrm{L}$ are the lateral dimension of the region enclosing the hydrophobic solvent (as shown in left diagram of Fig. 5.4 ) and $A_{\phi}$ is the area of the monolayer at zero tension. Since the tensionless simulations of planar bilayers revealed that the two chain architectures considered in that case had the same cross sectional area, $A_{\phi}$ turned out to be a composition-independent quantity. However, the host and guest lipid species in the present analysis, may well have different intrinsic areas, therefore resulting in a composition-dependent area of the final, mixed monolayer. To account for this composition dependence we will consider

$$
A_{\phi}=A_{0}-\epsilon \phi .
$$

The coefficients $\kappa_{m}$ and $\kappa_{A}$ in Eq 5.2 are the monolayer bending rigidity and area compressibility, $\mathrm{C}_{\phi}$ is its composition-dependent spontaneous curvature, and $A=2 \pi R L$ and $R$ are, respectively, its surface area and radius of curvature, defined at the center of mass of the lipids polar head-groups, as it is shown in 


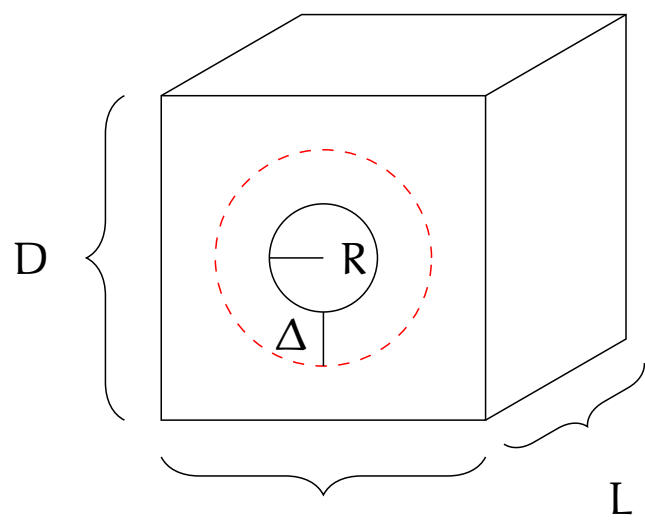

L

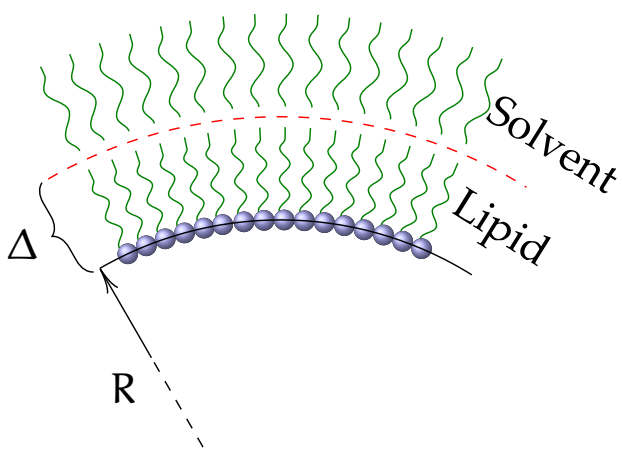

$\mathrm{D}$

Figure 5.4: Schematic representation of a single inverted monolayer surrounded by an hydrophobic incompressible solvent.

the right schematics of Fig.5.4. These last two quantities can be naturally related to the lateral dimensions of the enclosing volume via the incompressibility condition of both the hydrophobic solvent and the lipid tails. Under this condition, the volume occupied by the dense hydrophobic liquid is given by

$$
\mathrm{V}_{\mathrm{occ}}=\mathrm{D}^{2} \mathrm{~L}-\pi(\mathrm{R}+\Delta)^{2} \mathrm{~L},
$$

where $\Delta$ is the distance between the monolayer curvature plane and the tailhydrophobic solvent, which may well lie within the monolayer hydrophobic region, because of the affinity between the hydrophobic solvent and the lipid tails (like in a wet brush). Therefore, the dependence of the monolayer radius on the lateral dimensions of the system, will be given by

$$
R(D, L)=-\Delta+\frac{1}{\sqrt{\pi}}\left[D^{2}-V_{o c c} L^{-1}\right]^{1 / 2} \text {. }
$$

This relation provides a straightforward way to evaluate the pressure components parallel and perpendicular to the cylinder axis, which can be defined as

$$
\mathrm{P}_{\|}=-\frac{1}{\mathrm{D}^{2}}\left(\frac{\partial \mathrm{F}}{\partial \mathrm{L}}\right)_{\mathrm{D}} \quad \text { and } \quad \mathrm{P}_{\perp}=-\frac{1}{2 \mathrm{DL}}\left(\frac{\partial \mathrm{F}}{\partial \mathrm{D}}\right)_{\mathrm{L}} .
$$

Using Eq 5.2, these pressure components result in

$$
\begin{aligned}
& P_{\|}=-\frac{2 \pi R}{D^{2}} E_{R}-\frac{V_{o c c}}{D^{2} L(R+\Delta)}\left[E_{R}-\frac{\kappa_{m}}{R}\left(R^{-1}-C_{\phi}\right)\right], \\
& P_{\perp}=-\frac{1}{R+\Delta}\left[E_{R}-\frac{\kappa_{m}}{R}\left(R^{-1}-C_{\phi}\right)\right],
\end{aligned}
$$


where we have defined

$$
E_{R}=\frac{K_{m}}{2}\left(R^{-1}-C_{\phi}\right)^{2}+\frac{K_{A}}{A_{\phi}}\left(2 \pi R L-A_{\phi}\right) .
$$

Therefore, measuring the pressure components and the monolayer radius in systems with different lateral dimensions and compositions, provides an alternative way to evaluate the intrinsic curvatures of the individual lipids species, $\mathrm{C}_{+}$and $\mathrm{C}_{-}$, which are encoded in the definition of the monolayer spontaneous curvature

$$
\mathrm{C}_{\phi}=\mathrm{C}_{0}+\lambda^{\prime} \phi .
$$

Notice that this definition of $\lambda^{\prime}$ differs from the one given in Eq.3.14, where an additional prefactor was included in order to recover the standard coupling term between curvature and composition in the expression for the free energy of the bilayer, i.e., last term in $\mathrm{Eq} 3.23$.

\subsection{Simulation of inverted cylinders}

In order to test the ideas discussed in the previous sections, we have performed simulations of inverted lipid cylinders immersed in an hydrophobic solvent. One of the lipid architectures used in these simulations is the most cylindrical lipid from our simulations with planar bilayers, i.e., it comprises 4 hydrophilic beads in the head-group and 12 hydrophobic beads in the tail, resulting in an intrinsic spontaneous curvature $\mathrm{C}_{-}=0.072 \mathrm{~nm}^{-1}$. These lipids are used as the minority component (or guest species). For the second lipid species, we consider the same 16-bead long, linear architecture, but with 3 beads on the head-group and 13 beads on the tail. Having an inverted cone-shaped geometry, these lipids will comprise the host or inverted aggregate.

As it has already been discussed, the proper characterization of curvature of these inverted phases can only be achieved if the incompatibility between lipid species is low. For this reason, the virial coefficient between unlike head-group beads has been set equal to that of alike head-group beads. Specifically, we have set $v_{\mathrm{BC}}=v_{\mathrm{BB}}=v_{\mathrm{CC}}=0.1$ in Eq2.10. Finally, for the molecules comprising the solvent, we have replaced the head-group beads of the lipid structure by tail beads, therefore resulting in chains with 16 beads, whose hydrophobic properties are identical to those of the lipid tails.

All simulation in this study were done in the canonical ensemble, where the volume and number of particles in the system remain fixed. For all cases, we considered a mixture of 750 hydrophobic chains and 250 lipids. For these 250 
lipid, four composition ratios between the two lipid species were considered, namely $\phi=\{1.0,0.8,0.6,0.4\}$, where

$$
\phi=\frac{n_{\text {host }}-n_{\text {guest }}}{n_{\text {host }}+n_{\text {guest }}} .
$$

Here $n_{\text {host }}$ and $n_{\text {guest }}$ are the total number of host and guest lipids in the system.

In order to change the pressure of the system and the radius of curvature of the inverted monolayer, we varied the length of the simulation box parallel to the cylinder axis, $\mathrm{L}$, while letting fixed its cross sectional area, $\mathrm{D}^{2}=19.0^{2}$. Snapshots of the simulated cylinders for different compositions are presented in Fig 5.5 .

The first part of the analysis consisted in identifying the geometrical constants characterizing the hydrophobic region. This was done by measuring the monolayer radius for different lengths, L, and then fitting Eq 5.5 to these data. These results are shown in Fig 5.6, where different colors correspond to simulations with different compositions and the fit of Eq 5.5 is shown by the continuous line. The characteristics of the hydrophobic region extracted from this fit are $V_{\text {occ }}=1632.35 \mathrm{r}_{\mathrm{c}}^{3}$ and $\Delta=0.94 \mathrm{r}_{\mathrm{c}}$. These results verify that the volume occupied by the hydrophobic region is composition-independent. Furthermore, the fact that $\Delta$ is smaller than the thickness of a planar monolayer, $t_{m}=3.07 r_{c}$, denotes the interdigitation between the lipid tails and the hydrophobic solvent. This interdigitation is facilitated by the spreading of the lipid tails induced by curvature, and its net effect is to homogenize the hydrophobic region of the

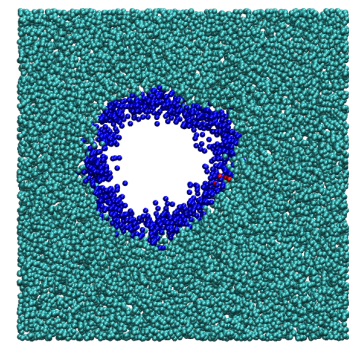

(a) $\phi=1.0$

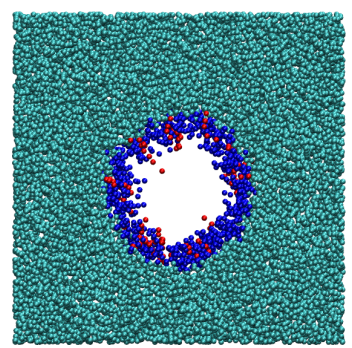

(b) $\phi=0.8$

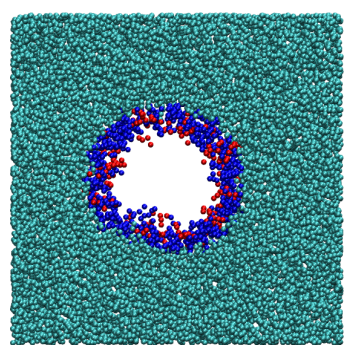

(c) $\phi=0.6$

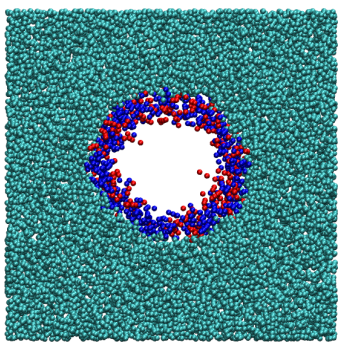

(d) $\phi=0.4$

Figure 5.5: Snapshots from simulations of inverted cylinders immersed in an hydrophobic solvent, for different compositions of the guest lipid species. The hydrophilic head-groups of the host lipid species are shown in blue and the head-groups of the guest species are shown in red. Tails of both lipid species and the hydrophobic solvent are shown in green. In all cases, the virial coefficients controlling the interactions between like and unlike head-group beads, have been set to 0.1 . 


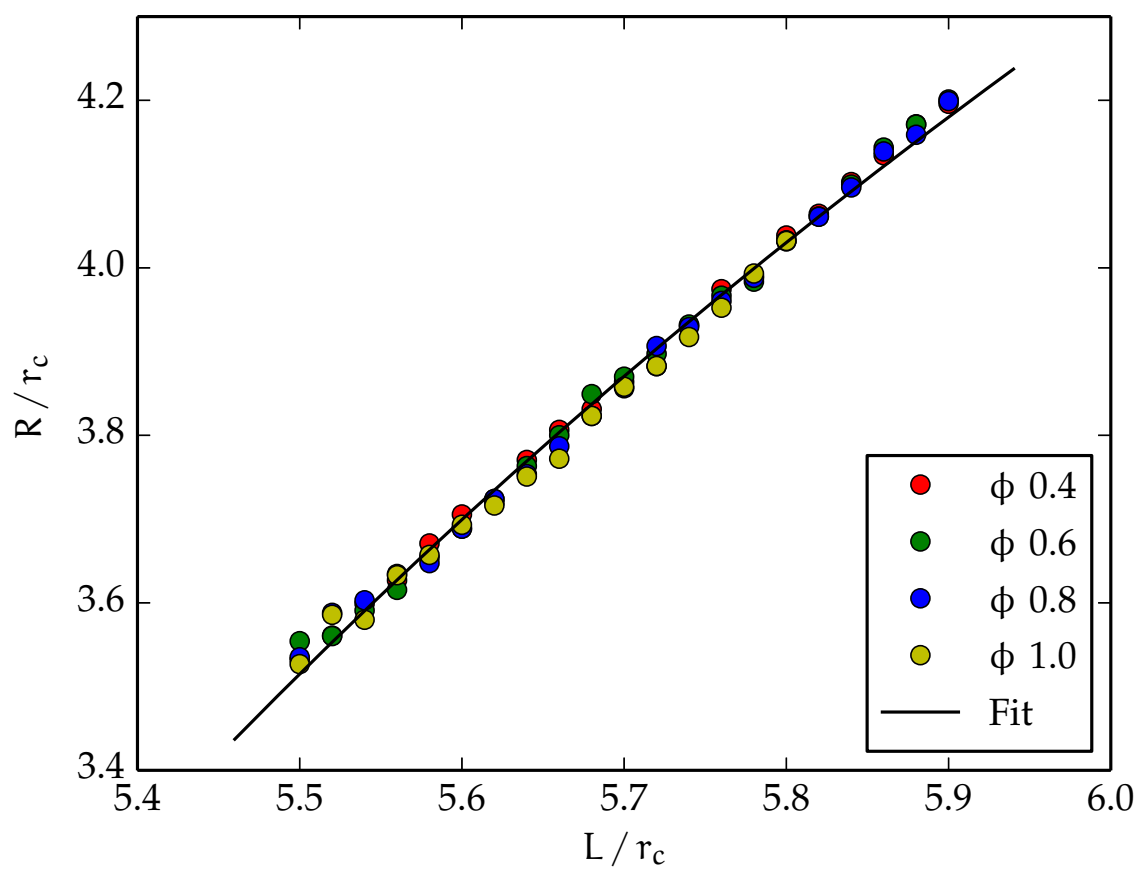

Figure 5.6: Radius of the inverted cylinder monolayers vs cylinder length. Result from simulations with different compositions are shown in color code and the fit of Eq 5.5 corresponds to the continuous line.

monolayer. This is consistent with our previous investigations of the density profile of planar bilayers, Fig 4.5, where it was found that the difference between the hydrophilic peak-to-peak distance and the hydrophobic full width at half maximum was $\sim 1.7 \mathrm{r}_{\mathrm{c}}$, therefore resulting in a hydrophobic-hydrophilic interface width $\sim 0.85 \mathrm{r}_{\mathrm{c}}$, on each monolayer.

During the simulations, the pressure components parallel and perpendicular to the cylinder axis, were evaluated from the virial. Then, having characterized the cylinder radius as a function of its length, Eq.5.7 was used to fit the parallel pressure from simulation with different compositions. The graphs of these fits are shown in Fig 5.7 and the parameters obtained from them are compiled in Tab.5.1. The first remarkable feature of these results is that the proportionality constant between the tensionless area, $A_{\phi}$, and composition is negative, thus reflecting that the intrinsic area of the inverted-cone lipid species is larger than that of the cylindrical species. This may be rationalized by noticing that tails in the former case are one bead longer than those in the latter one. Therefore, tails of the inverted lipids will spread over broader areas, consistently with the interdigitation picture just described. 


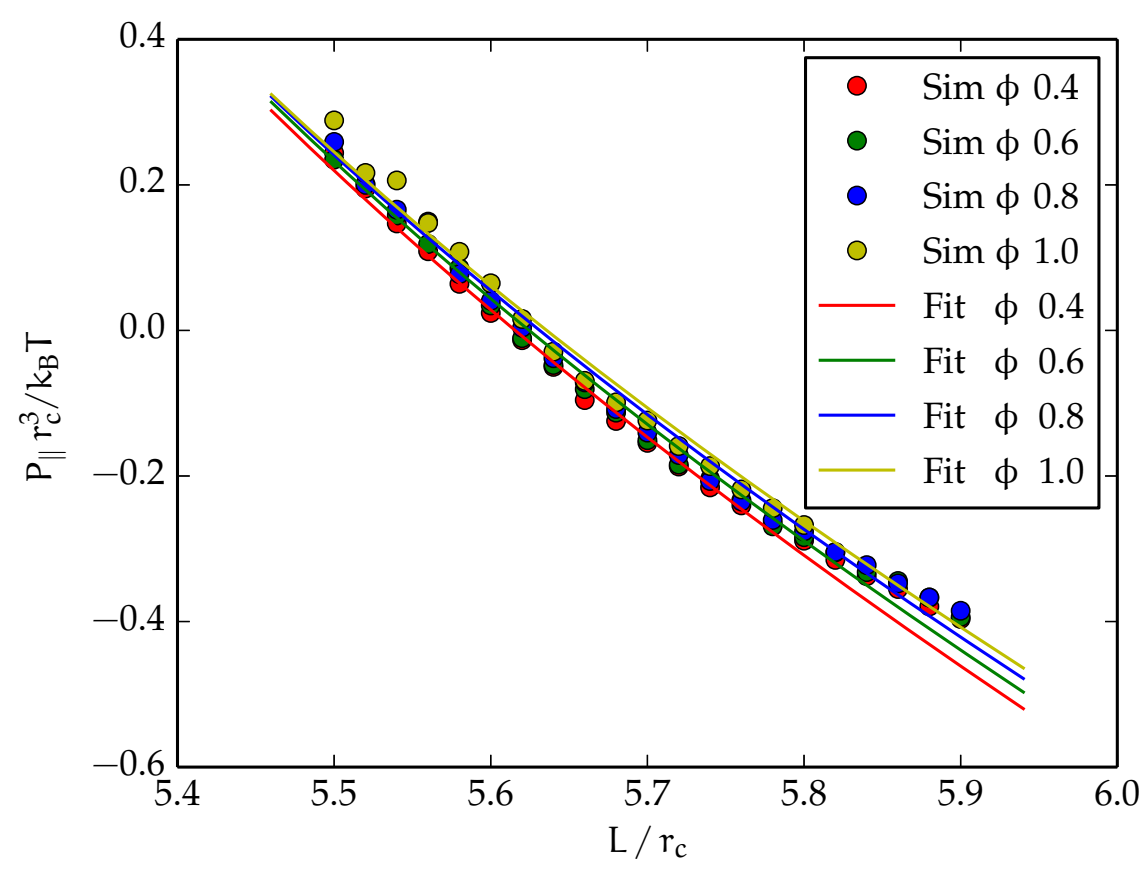

Figure 5.7: Parallel pressure vs cylinder length. Results from simulations are shown as dots and the corresponding fits of Eq 5.7 are shown as the continuous line. Results from simulations with different compositions are distinguished by color.

Another remarkable result is that the bending rigidity of these inverted cylinders is similar to the one obtained in the simulations with bilayer membranes $\left(\mathrm{K}_{\mathrm{m}}=10.6 \mathrm{k}_{\mathrm{B}} \mathrm{T}\right)$. This is in good agreement with the aforementioned idea that the bending rigidity is left practically unaffected by both composition and curvature. A different behavior is observed for the area compressibility of the inverted monolayers, $\mathrm{K}_{\mathrm{A}}$, whose value is almost a third of that obtained for bilayer membranes. Possible reasons for this effect are the gain of configurational entropy of the lipids tails in the curved geometry and the mixing of tails and the hydrophobic solvent, which would make the whole hydrophobic region more prone to fluctuate.

The values of $\lambda^{\prime}$ and $C_{0}$ obtained from these simulations result in intrinsic spontaneous curvatures $\mathrm{C}_{+}=0.45$ and $\mathrm{C}_{-}=0.11$ for the inverted and cylindrical lipids species, respectively. In this case, the spontaneous curvature of the cylindrical lipid species is slightly overestimated, when compared to the result obtained from the analysis with bilayers, although still asserting that its geometrical configuration is highly cylindrical. A possible explanation for this curvature increase is that the natural tendency of the inverted configuration 
CHAPTER 5. SPONTANEOUS CURVATURE FROM INVERTED LIPID STRUCTURES

Table 5.1: Set of parameters obtained from the fits of Eq.5.7 and Eq.5.5 to the cylinder radius and parallel pressure measured from simulations.

\begin{tabular}{lclr}
\hline \hline Quantity & Symbol & Unit & Value \\
\hline Hydrophobic incompressible volume & $\mathrm{V}_{\mathrm{occ}}$ & $\mathrm{r}_{\mathrm{c}}^{3}$ & 1632.35 \\
Hydrophobic-hydrophilic interface width & $\Delta$ & $\mathrm{r}_{\mathrm{C}}$ & 0.94 \\
Area-composition proportionality constant & $\epsilon$ & $\mathrm{r}_{\mathrm{c}}^{2}$ & -10.52 \\
Tensionless area of a symmetric mixed leaflet & $\mathrm{A}_{0}$ & $\mathrm{r}_{\mathrm{c}}^{2}$ & 132.05 \\
Monolayer bending rigidity & $\mathrm{K}_{\mathrm{m}}$ & $\mathrm{k}_{\mathrm{B}} \mathrm{T}$ & 11.06 \\
Monolayer area compressibility & $\mathrm{K}_{\mathrm{A}}$ & $\mathrm{k}_{\mathrm{B}} \mathrm{T} / \mathrm{r}_{\mathrm{c}}^{2}$ & 11.82 \\
Intrinsic curvature-composition coupling & $\lambda^{\prime}$ & $\mathrm{r}_{\mathrm{c}}^{-1}$ & 0.17 \\
Average curvature of the two lipid species & $\mathrm{C}_{0}$ & $\mathrm{r}_{\mathrm{C}}^{-1}$ & 0.28 \\
\hline \hline
\end{tabular}

to create voids at the end of the monolayer hydrophobic region, will promote the interdigitation of solvent particles between the lipid tails. This interdigitation would act as a spacer between tails that will ultimately result in an effective curvature increase. This effect could be accounted for by the inclusion of a curvature-dependent term in the expression for the monolayer area at zero tension, Eq 5.3. Furthermore, the addition of this term could help to improve the quality of the fit. 


\section{Chapter 6}

\section{Summary and outlook}

In the fluid-mosaic model, lipids and proteins of the plasma membrane were conceived as a passive fluid whose only function was to isolate the cell from its environment. Since its introduction in 1972, the model has been further extended and, today, the plasma membrane is depicted as an active platform that regulates and gives support to many cellular processes. To a greater or lesser extent, this regulatory character of the plasma membrane, has to be dictated by the properties of its constituent lipids. In particular, the interplay between curvature and composition in lipid bilayers can drastically influence the lateral organization of lipids in the membrane. For instance, recent studies suggest that an intrinsic coupling between the local curvature and composition in lipid bilayers may give rise to the formation of "rafts", i.e., spatially modulated phases or microemulsion-like domains of phospholipids and cholesterol, whose properties differ from their surroundings. There is evidence that these sub-micrometer, highly dynamic and transient structures are involved in many membrane-mediated biological processes. In particular, they are thought to compartmentalize such processes to a given area in the cell membrane and stabilize larger platforms through protein-protein and protein-lipid interactions. Additionally, this coupling provides a physical mechanism to explain the composition asymmetry between inner and outer leaflets observed in bilayers under strong curvature deformations, as those in tube-pulling experiments.

In this work, we have devised computer simulation techniques to study the interplay between the local curvature and composition in mixed lipid bilayers. In Chapter 2, we have presented an extension of the implicit-solvent, coarsegrained model by Hömberg et al. This extension was necessary to study the physical properties of self-assembled lipid structures with more than one lipid species. Specifically, we have included additional terms in the non-bonded Hamiltonian of the system, which beside accounting for the interactions between the lipids' hydrophobic and hydrophilic blocks, also account for the re- 
pulsion between unlike head-group species. These additional terms, however, can be mapped to physical quantities that are easily accessible in experiments. Therefore, our extension has left intact the simplicity and beauty of the original model.

Since the relaxation of composition fluctuations is a diffusion-mediated process, the simulation of mixed systems in the canonical ensemble result in a very computationally inefficient process, specially when the system is in the close proximity of the demixing critical point. Because of this, most of the simulations in this work were carried out in the semi-grand canonical ensemble. This ensemble was implemented as an hybrid approach were the equations of motion where integrated by standard molecular dynamics techniques and, additionally, Monte Carlo moves were used to mutate one lipid species into another, based on an externally imposed difference in chemical potential between the lipid species. The simulations were further optimized via the domain-decomposition, parallelization technique. This parallel implementation showed a reasonable scaling up to 64 cores, therefore allowing us to access the diffusion regime of large systems (typically spanning $\sim 10^{5}$ particles).

In Chapter 3, we introduced a new model describing the thermodynamics of mixing in the two monolayers of a membrane composed by lipid species with different spontaneous curvatures. The novelty of this model is that, beside incorporating the intrinsic coupling between the local curvature and composition fields, as well as contributions from the free energy of mixing on each monolayer, it also accounts for the local curvature difference between the two apposing membrane leaflets. We pointed out that this last contribution is particularly important for membranes whose local curvature modulations are comparable to the inverse bilayer thickness. One of the main predictions of this model is that the maximum composition difference across the membrane, which is compatible with the average composition between the two monolayers (recall that $\phi \in[-1+|\psi|, 1-|\psi|])$, is reached at a specific curvature. In other words, there exist a "saturation curvature" for which the composition difference between the apposing monolayers is maximal. We have also shown that the occurrence of this saturation curvature will depend on the segregation conditions of the system. In particular, for low incompatibility between lipid species, the theoretical saturation curvature may be well beyond the inverse bilayer thickness, a quantity that sets a limit on the physical curvatures accessible to the system. In contrast, saturation conditions in strongly segregated systems can be reached at small curvatures, such as those induced in planar bilayers by the effect of thermal fluctuations.

Far from saturation, our phenomenological mean-field model predicts a linear relation between the equilibrium composition difference and membrane lo- 
cal curvature, where the proportionality term is what we denoted as the "effective curvature-composition coupling". We have shown that this quantity depends on the geometric and elastic properties of the bilayer, as well as the incompatibility between lipid species and the average composition of the system.

In the last section of Chapter 3, we have investigated possible further contributions to the lipid sorting, due to a dependence of the bending rigidity on curvature. There, we showed that the qualitative behavior just described remains unchanged by this additional contribution. Furthermore, we saw that even for highly-curved and strongly segregated systems, the quantitative corrections are so small that can be effectively neglected.

In Chapter 4, we presented the results of bilayer membranes simulations. The first two sections were devoted to the parametrization of the coarse-grained model and the evaluation of the non-free parameters in the mean-field description. Several conclusion were drawn from these measurements: the characterization of the system average composition as a function of the imposed difference in chemical potential between the two lipids species aids to identify the two segregation conditions used in this study. In particular, the diverging susceptibility of composition fluctuations for the high incompatibility case revealed that those systems are in the close vicinity of the demixing critical point, but still within the mixed phase. Another result, is that the particular choice of chain architectures for this study results in lipid species that self-assemble into planar bilayers with similar cross-sectional areas and longitudinal extension. Nevertheless, a quick glance at lateral pressure profile across the bilayer, suggested that the intrinsic spontaneous curvature of both species are, indeed, different. A final remarkable conclusion from this analysis is that the power spectra of shape fluctuations do not seem to be influenced neither by composition nor the segregation conditions of the system. Since curvature and composition are related via the effective curvature-composition coupling, this last finding is in good agreement with the negligible corrections to the lipid sorting due to a curvature dependent bending rigidity.

In the third section of Chapter 4 we analyzed the interplay between curvature and composition of planar bilayers, in terms of the phenomenological mean-field model. Conclusions from this analysis can be summarized as follows:

- Low incompatibility results in a weaker coupling, since the elastic energy gain when sorting lipids into curvature-favored regions cannot overcome the concomitant entropy loss.

- High incompatibility results in much larger effects where, in addition to the intrinsic coupling, the curvature dependence of the free energy of mixing contributes to the lateral sorting of lipids. This combined effect results 
in a higher coupling that is further enhanced as the system approaches the demixing critical point.

- The quantitative comparison between the phenomenological model and the computer simulations demonstrates that a single set of parameters can consistently describe the behavior of mixed membranes for different average compositions and incompatibilities.

- The spontaneous curvatures of the lipids species used in this study are in good agreement with those reported for biologically relevant lipids.

Finally, since the linear relationship between curvature and the composition difference across the bilayer holds only far from saturation and since this assumption is expected to break down for highly-curved and strongly segregated systems, we carried out simulation with cylinders of different radius and for both incompatibility cases. From these simulations we conclude that the implicit relation between curvature and composition, Eq 3.24. duly accounts for the additional entropy of mixing contributions.

An alternative method to measure the intrinsic curvature of lipids was presented in Chapter 5. In the first section, a brief review of the structures resulting from the self-assembly of inverted-cone shaped lipids was presented. It has been argued that, in the absence of tension or additional packing constraints, the curvature of these inverted and single-monolayer aggregates coincides with the spontaneous curvature of their constituting lipids. This feature offers an immediate mechanism to measure the spontaneous curvature of, for instance, cylindrical or cone-shaped lipids, since the addition of these guest lipids will induce the expansion or contraction of the mixed aggregate, which will be proportional to the concentration of guest lipids. However, it has been seen that if the two lipid species do not mix well, the concomitant segregation of the guest species may induce significant deviations from the cylindrical geometry that will ultimately make the characterization of the system curvature infeasible. Furthermore, even when the lipid species mix well, the system anisotropy due to the stretching of the lipid tails sitting at the "voids" between different inverted structures (where significant packing frustration occurs), may still have a large influence on the lateral organization of lipids.

In the second section, it was suggested to allow the self-assembly of inverted cylindrical monolayers within an hydrophobic solvent with the same hydrophobic properties than the lipid tails. Studying the thermodynamics of these immersed cylinders, a relationship between the pressure components of the solvent + monolayer system and the geometric characteristics of the monolayer were found. Finally, the chapter closes with the analysis of such immersed worm-like micelles. The main conclusions that result from this alterna- 
tive method are in agreement with those obtained from the analysis with bilayer membranes. However, in this case there may be additional contributions to the free energy of the systems accounting for an increase of the tensionless area of a mixed monolayer, due to the interdigitation of solvent molecules between lipid tails, needed to prevent the formation of voids.

A natural extension of this project would be to investigate the influence of the curvature-composition coupling on the dynamical properties of the systems studied so far. For instance, beside enhancing the complementary sorting of lipids across the membrane, the emergence of packing effects on curved membranes may drastically influence the lateral diffusion of their constituting lipids. In particular, for membrane tubes such as those studied in Chapter 4, one would expect that the more constrained environment experienced by lipids in the inner leaflet, will result in a slower diffusion than that of lipids in the outer one. Nevertheless, preliminary results from the temporal evolution of the lipids mean square displacement (MSD) in the inner and outer leaflets of bilayer tubes with different midplane radii, does not exhibit a significant curvature dependence for the total MSD along the cylinder surface (in-plane), as shown in Fig.6.1.

In the short-time limit, the in-plane MSD exhibits the typical ballistic behavior, where particles diffuse freely, i.e., in the absence of interactions. Once that particles had diffused a mean free path comparable to the cutoff radius of nonbonded interactions, $r_{c}$, they start to notice the presence of neighboring particles, through collisions which drastically alter their velocities. It is at that very moment when one would expect packing effects to come into play, modifying the frequency with which collisions occur: particles in the inner leaflet will collide more frequently than those in the outer leaflet, since they will experience a denser or more constraint environment, therefore resulting in an effectively reduced mobility, i.e., after that time, dynamics in the outer leaflet will be faster than in the inner one. This effect should become more pronounced as the midplane radius curvature is decreased. A possible explanation for the absence of this effect in the preliminary simulations may be attributed to the softness of the implicit-solvent coarse-grained model and, perhaps, a fine-tuning of the virial coefficients setting the incompressibility of the dense hydrophobic region may be all that is required. Of course, such re-parametrization will have influence on the lipid-sorting results presented in this work. However the qualitative equilibrium features presented here, should be left unchanged, since making the tails more incompressible may only lead to a enhancement of the interplay between curvature and composition.

In conclusion, this work has investigated the interplay between curvature and composition in binary mixture lipid structures, with different topologies (planar membranes, cylindrical bilayers and reverse worm-like micelles) and 
over a broad range of curvatures. Furthermore, two qualitatively different segregation regimes have been considered. Concerning the analysis of the bilayer structures, a new phenomenological mean-field model has been proposed to describe their thermodynamics of mixing. The novelty of this model is that beside considering contributions from the free energy of mixing of the individual monolayers, it also considers their difference in curvature. It has been shown that these two features account for entropic contributions to the lateral sorting of lipids that become particularly important when the local membrane shape modulations are comparable to the bilayer thickness. Based on this model, a new methodology to measure the intrinsic curvature of the two lipid species comprising the bilayer has been proposed. The spontaneous curvatures obtained from this analysis have been found to be in good agreement with those obtained from a commonly employed experimental technique, where lipids with unknown curvature are inserted into an inverted structure whose macroscopic curvature coincides with the intrinsic curvature of its constituting lipids, therefore validating the proposed new method.

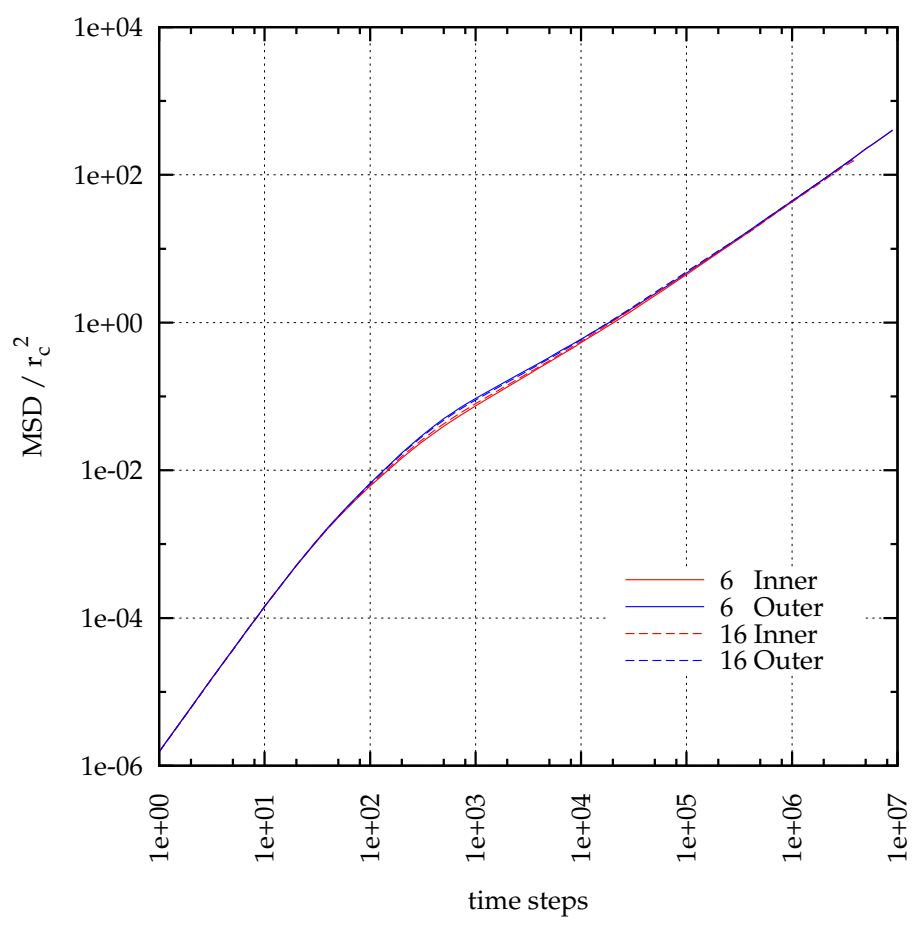

Figure 6.1: Mean square displacement (MSD) of lipids in bilayer tubes. Results for tubes with midplane radii $R=6.0 r_{c}$ and $R=16.0 r_{c}$ are shown by continuous and dashed lines, respectively. Results for the inner leaflet are displayed in red and result from the outer leaflet are shown in blue. 


\section{Bibliography}

[1] S. L. Miller, Science 117, 528 (1953).

[2] S. J. Singer and G. L. Nicolson, Science 175, 720 (1972).

[3] S. Mishra and P. G. Joshi, Journal of Neurochemistry 103, 135 (2007).

[4] S. Semrau and T. Schmidt, Soft Matter 5, 3174 (2009).

[5] E. Li, , and K. Hristova, Biochemistry 45, 6241 (2006).

[6] M. Z. Gilcrease, Cancer Letters 247, 1 (2007).

[7] D. J. Triggle, in , edited by J. F. Danielli, A. C. Riddiford, and M. D. Roenberg (Elsevier, 1970), vol. 3 of Recent Progress in Surface Science, pp. 273290.

[8] K. Simons and E. Ikonen, Nature 387, 569 (1997).

[9] A. R. Honerkamp-Smith, S. L. Veatch, and S. L. Keller, Biochimica et Biophysica Acta - Biomembranes 1788, 53 (2009).

[10] S. A. Safran, P. A. Pincus, D. Andelman, and F. C. MacKintosh, Physical Review A 43, 1071 (1991).

[11] R. Brewster and S. A. Safran, Biophysical Journal 98, L21 (2010).

[12] A. Yethiraj and J. C. Weisshaar, Biophysical Journal 93, 3113 (2007).

[13] T. Fischer and R. L. C. Vink, Journal of Physics: Condensed Matter 22, 104123 (2010).

[14] M. Schick, Physical Review E 85, 031902 (2012).

[15] R. Goetz and R. Lipowsky, Journal of Chemical Physics 108, 7397 (1998).

[16] H. Noguchi and M. Takasu, Physical Review E 64, 041913 (2001). 
[17] R. G. Larson, L. E. Scriven, and H. T. Davis, Journal of Chemical Physics 83, 2411 (1985).

[18] B. Smit, A. G. Schlijper, L. A. M. Rupert, and N. M. V. Os, Journal of Physical Chemistry 94, 6933 (1990).

[19] M. M. Kamala, D. Mills, M. Grzybeka, and J. Howard, Proceedings of the Royal Society of London Series A 106, 22245 (2009).

[20] S. A. Safran, Statistical thermodynamics of surfaces, interfaces, and membranes (Addison-Wesley Reading, MA, 1994).

[21] J. N. Israelachvili, Intermolecular and surface forces (Academic Press, 1991), 2nd ed.

[22] E. Lindahl and O. Edholm, Journal of Chemical Physics 113, 3882 (2000).

[23] D. H. Tsai, Journal of Chemical Physics 70, 1375 (1979).

[24] J. N. Israelachvili, D. J. Mitchell, and B. W. Ninham, Journal of the Chemical Society, Faraday Transactions 2: Molecular and Chemical Physics 72, 1525 (1976).

[25] M. A. Lemmon, Traffic 4, 201 (2003).

[26] V. A. Frolov, A. V. Shnyrova, and J. Zimmerberg, Cold Spring Harbor Perspectives in Biology 3 (2011).

[27] N. Kahya, D. Scherfeld, K. Bacia, and P. Schwille, Journal of Structural Biology 147, 77 (2004).

[28] J. Korlach, P. Schwille, W. W. Webb, and G. W. Feigenson, Proceedings of the National Academy of Sciences 96, 8461 (1999).

[29] M. D. Collins and S. L. Keller, Proceedings of the National Academy of Sciences 1052, 124 (2008).

[30] A. Tian, B. R. Capraro, C. Esposito, and T. Baumgart, Biophysical Journal 97, 1636 (2009).

[31] T. Baumgart, A. T. Hammond, P. Sengupta, S. T. Hess, D. A. Holowka, B. A. Baird, and W. W. Webb, Proceedings of the National Academy of Sciences 104, 3165 (2006).

[32] T. Baumgart, S. T. Hess, and W. W. Webb, Nature 425, 821 (2003). 
[33] A. Callan-Jones, B. Sorre, and P. Bassereau, Cold Spring Harbor Perspectives in Biology 3 (2011).

[34] R. Parthasarathy, C. han Yu, , and J. T. Groves, Langmuir 22, 5095 (2006).

[35] D. Marquardt, B. G. Barbara, and G. Pabst, Membranes 5, 180 (2015).

[36] M. Hömberg and M. Müller, Journal of Chemical Physics 132, 155104 (2010).

[37] N. Attig, K. Binder, H. Grubmüller, and K. Kremer, eds., Computational soft matter: from synthetic polymers to proteins; NIC winter school, 29 February 6 March 2004, Gustav-Stresemann-Institut, Bonn, Germany - Lecture Notes, vol. 23 of NIC series (FZJ, John von Neumann Institute for Computing, Jülich, 2004).

[38] M. P. Allen and D. J. Tildesley, Computer simulation of liquids, Oxford science publications (Oxford University Press, 1989), reprint ed.

[39] P. Schuster, Berichte der Bunsengesellschaft für physikalische Chemie 87, 291 (1983).

[40] A. J. Stone, The theory of intermolecular forces (Clarendon Press, Oxford, 1996).

[41] S. L. Price, in Reviews in Computational Chemistry (John Wiley \& Sons, Inc., 2007), pp. 225-289.

[42] P. Ilg, V. Mavrantzas, and H. C. Öttinger, Multiscale modeling and coarse graining of polymer dynamics: simulations guided by statistical beyondequilibrium thermodynamics (Wiley-VCH Verlag GmbH \& Co. KGaA, 2010), pp. 343-383.

[43] D. R. Heine, A. R. Rammohan, and J. Balakrishnan, Molecular Simulation 33, 391 (2007).

[44] A. Polley, S. Vemparala, and M. Rao, Journal of Physical Chemistry B 116, 13403 (2012).

[45] J. T. Kindt, Molecular Simulation 37, 516 (2011).

[46] C. Lubich, From quantum to classical molecular dynamics: reduced models and numerical analysis, Zurich lectures in advanced mathematics (European Mathematical Society, 2008). 
[47] S. Grimme, Journal of Chemical Theory and Computation 10, 4497 (2014).

[48] Y. Duan, C. Wu, S. Chowdhury, M. C. Lee, G. Xiong, W. Zhang, R. Yang, P. Cieplak, R. Luo, T. Lee, et al., Journal of Computational Chemistry 24, 1999 (2003).

[49] M. Müller, K. Katsov, and M. Schick, Physics Reports 434, 113 (2006).

[50] P. Español, Statistical mechanics of coarse-graining (Springer Berlin Heidelberg, 2004), vol. 640 of Lecture Notes in Physics, pp. 69-115.

[51] R. Bradley and R. Radhakrishnan, Polymers 5, 890 (2013).

[52] S. J. Marrink, A. H. de Vries M., and A. E. Mark, Journal of Physical Chemistry B 108, 750 (2004).

[53] P. de Gennes, Scaling concepts in polymer physics (Cornell University Press, 1979).

[54] J. Kirkwood and F. P. Buff, Journal of Chemical Physics 19, 774 (2013).

[55] M. Born and H. S. Green, Proceedings of the Royal Society of London Series A 188, 10 (1946).

[56] R. Zwanzig, Physical Review 124, 983 (1961).

[57] R. Zwanzig, Non-equilibrium statistical mechanics (Oxford University Press, 2001).

[58] H. Mori, Progress of Theoretical Physics 33, 423 (1965).

[59] S.-K. Ma, Statistical mechanics (World Scientific, Philadelphia, 1985).

[60] W. Tschöp, K. Kremer, J. Batoulis, T. Bürger, and O. Hahn, Acta Polymerica 49, 61 (1998).

[61] D. Reith, M. Pütz, and F. Müller-Plathe, Journal of Computational Chemistry 24,1624 (2003).

[62] D. S. Frenkel and B. Smit, Understanding molecular simulation: from algorithms to applications, Computational science series (Academic Press, San Diego, San Francisco, New York, 2002).

[63] R. L. Henderson, Physics Letters A 49, 197 (1974).

[64] F. Ercolessi and J. B. Adams, Europhysics Letters 26, 583 (1994). 
[65] L. Lu, J. F. Dama, and G. A. Voth, The Journal of Chemical Physics 139, 121906 (2013).

[66] F. Müller-Plathe, European Journal of Chemical Physics and Physical Chemistry 3, 754 (2002).

[67] A. Gorban, N. Kazantzis, Y. Kevrekidis, H. C. Öttinger, and C. Theodoropoulos, eds., Model reduction and coarse-graining approaches for multiscale phenomena (Springer, Berlin-Heidelberg-New York, 2006).

[68] G. A. Voth, ed., Coarse-graining of condensed phase and biomolecular systems (CRC Press / Taylor and Francis Group, Boca Raton, FL, 2009).

[69] I. R. Cooke and M. Deserno, Journal of Chemical Physics 123, 224710 (2005).

[70] F. Schmid, D. Düchs, O. Lenz, and B. West, Computer Physics Communications 177, 168 (2007), proceedings of the conference on computational physics 2006.

[71] S. J. Marrink, H. J. R., S. Yefimov, D. P. Tieleman, and A. H. de Vries, Journal of Physical Chemistry B 111, 7812 (2007).

[72] I. Carmesin and K. Kremer, Macromolecules 21, 2819 (1988).

[73] A. Christiansson, F. A. Kuypers, B. Roelofsen, J. A. O. den Kamp, and L. L. van Deenen, Journal Cell Biology 101, 1455 (1985).

[74] S. M. Gruner, Proceedings of the National Academy of Sciences of the United States of America 82, 3665 (1985).

[75] A. A. Efimova, A. V. Sybachin, and A. A. Yaroslavov, Polymer Science Series C 53, 89 (2011).

[76] N. Fuller and R. P. Rand, Biophysical Journal 81, 243 (2001).

[77] S. Leikin, M. M. Kozlov, N. L. Fuller, and R. P. Rand, Biophysical Journal 71, 2623 (1996).

[78] J. M. Drouffe, A. C. Maggs, and S. Leibler, Science 254, 1353 (1991).

[79] O. Farago, Journal of Chemical Physics 119, 596 (2003).

[80] Z.-J. Wang and D. Frenkel, Journal of Chemical Physics 122, 234711 (2005). 
[81] A. Srivastava and G. A. Voth, Journal of Chemical Theory and Computation 10, 4730 (2014).

[82] M. Hömberg, Ph.D. thesis, Georg-August University School of Science (GAUSS) (2011).

[83] R. B. Bird, C. F. Curtiss, R. C. Armstrong, and O. Hassager, Dynamics of polymeric liquids, kinetic theory (Volume 2) (Wiley-Interscience, 1987), volume 2 ed.

[84] M. Doi, Introduction to polymer physics (Oxford University Press, Oxford and New York, 1996).

[85] M. Rubinstein and R. Colby, Polymer physics (Oxford University Press, Oxford and New York, 2003).

[86] H. B. Callen, Thermodynamics (John Wiley \& Sons, Inc., New York, N.Y., 1960).

[87] M. L. Huggins, Journal of Chemical Physics 9 (1941).

[88] P. J. Flory, Journal of Chemical Physics 10, 51 (1942).

[89] P. de Gennes, Scaling concepts in polymer physics (Cornell University Press, 1979), 1st ed.

[90] P. Tarazona, Molecular Physics 52, 81 (1984).

[91] P. Tarazona, U. M. B. Marconi, and R. Evans, Molecular Physics 60, 573 (1987).

[92] L. Verlet, Physical Review 159, 98 (1967).

[93] M. Tuckerman, B. J. Berne, and G. J. Martyna, Journal of Chemical Physics 97, 1990 (1992).

[94] M. . Winger, D. Trzesniak, R. Baron, and W. F. van Gunsteren, Physical Chemistry Chemical Physics 11, 1934 (2009).

[95] P. H. Hünenberger, Thermostat algorithms for molecular dynamics simulations (Springer Berlin Heidelberg, 2005), vol. 173 of Advances in Polymer Science, pp. 105-149.

[96] C. P. Lowe, Europhysics Letters 47, 145 (1999). 
[97] E. A. Koopman and C. P. Lowe, Journal of Chemical Physics 124, 204103 (2006).

[98] H. C. Andersen, journal of Chemical Physics 72, 2384 (1980).

[99] S. Nosé, Journal of Chemical Physics 81, 511 (1984).

[100] W. G. Hoover, Physical Review A 31, 1695 (1985).

[101] P. J. Hoogerbrugge and J. M. V. A. Koelman, Europhysics Letters 19, 155 (1992).

[102] P. Español and P. Warren, Europhysics Letters 30, 191 (1995).

[103] R. D. Groot and P. B. Warren, Journal of Chemical Physics 107, 4423 (1997).

[104] I. Pagonabarraga, M. H. J. Hagen, and D. Frenkel, Europhysics Letters 42, 377 (1998).

[105] L.-J. Chen, Z.-Y. Lu, H.-J. Qian, Z.-S. Li, and C.-C. Sun, Journal of Chemical Physics 122, 104907 (2005).

[106] J. G. Kirkwood and F. P. Buff, Journal of Chemical Physics 17, 338 (1949).

[107] A. Kolb and B. Dünweg, Journal of Chemical Physics 111, 4453 (1999).

[108] D. P. Landau and K. Binder, A guide to Monte Carlo simulations in statistical physics (Cambridge University Press, 2009), 3rd ed.

[109] H. E. Stanley, Introduction to phase transitions and critical phenomena (international series of monographs on physics) (Oxford University Press, USA, 1987).

[110] K. Binder, ed., Monte Carlo and molecular dynamics simulations in polymer science (Oxford University Press, 1995), 1st ed.

[111] J. de Joannis, Y. Jiang, F. Yin, , and J. T. Kindt, Journal of Physical Chemistry B 110, 25875 (2006).

[112] S. Plimpton and B. Hendrickson, Journal of Computational Chemistry 17, 326 (1996).

[113] D. C. Rapaport, The art of molecular dynamics simulation (Cambridge University Press, 1995), 2nd ed.

[114] P. Canham, Journal of Theoretical Biology 26, 61 (1970). 
[115] W. Helfrich, Zeitschrift für Naturforschung 28, 693 (1973).

[116] C. W. Oseen, Transactions of the Faraday Society 29, 883 (1933).

[117] F. C. Frank, Discussions of the Faraday Society 25, 19 (1958).

[118] R. Lipowsky and S. Grotehans, Biophysical Chemistry 49, 27 (1994).

[119] R. Goetz, G. Gompper, and R. Lipowsky, Physical Review Letters 82, 221 (1999).

[120] E. Lindahl and O. Edholm, Biophysical Journal 79, 426 (2000).

[121] K. Bohinc, V. Kralj-Iglič., and S. May, The Journal of Chemical Physics 119, 7435 (2003).

[122] J.-B. Fournier, Europhysics Letters 43, 725 (1998).

[123] C. Cametti, F. D. Luca, A. D'Ilario, M. Macrì, G. Briganti, and B. Maraviglia, in Trends in Colloid and Interface Science $V$, edited by M. Corti and F. Mallamace (Steinkopff, 1991), vol. 84 of Progress in Colloid E Polymer Science, pp. 465-469.

[124] L. M. Loura and M. Prieto, in Fluorescent Methods to Study Biological Membranes, edited by Y. Mély and G. Duportail (Springer Berlin Heidelberg, 2013), vol. 13 of Springer Series on Fluorescence, pp. 71-113.

[125] H. Muddana, H. Chiang, and P. Butler, Biophysical Journal 102, 489 (2012).

[126] B. Kollmitzer, P. Heftberger, M. Rappolt, and G. Pabst, Soft Matter 9, 10877 (2013).

[127] R. Parthasarathy, C. han Yu, , and J. T. Groves, Langmuir 22, 5095 (2006).

[128] T.-Y. Yoon, C. Jeong, J. H. Kim, M. C. Choi, M. W. Kim, and S.-D. Lee, Applied Surface Science 238, 299 (2004).

[129] D. Diedrich and E. Cota-Robles, Journal of Bacteriology 119, 1006 (1974).

[130] P. Janmey and P. Kinnunen, Trends in Cell Biology 16, 538 (2006).

[131] Leibler, Journal de Physique 47, 507 (1986).

[132] T. Taniguchi, K. Kawasaki, D. Andelman, and T. Kawakatsu, Condensed Matter and Materials Communications 1, 75 (1993). 
[133] D. Andelman, T. Kawakatsu, and K. Kawasaki, Europhysics Letters 19, 57 (1992).

[134] E. W. Kaler, A. K. . Murthy, B. E. Rodriguez, and J. A. Zasadzinski, Science 245, 1371 (1989).

[135] S. A. Safran and F. C. MacKintosh, Physical Review E 47, 1180 (1993).

[136] U. Seifert, Physical Review Letters 70, 1335 (1993).

[137] S. May, Soft Matter 5, 3148 (2009).

[138] O. Edholm and J. F. Nagle, Biophysical Journal 89, 1827 (2005).

[139] E. Lindahl and O. Edholm, Biophysical Journal 79, 426 (2000).

[140] G. Brannigan and F. L. H. Brown, Journal of Chemical Physics 120, 1059 (2004).

[141] S. I. Mukhin and S. Baoukina, Physical Review E 71, 061918 (2005).

[142] J. Gullingsrud and K. Schulten, Biophysical Journal 86, 3496 (2004).

[143] K. Binder, in Theories and mechanism of phase transitions, heterophase polymerizations, homopolymerization, addition polymerization (Springer Berlin Heidelberg, 1994), vol. 112 of Advances in Polymer Science, pp. 181-299.

[144] H. Frielinghaus, D. Schwahn, K. Mortensen, L. Willner, and K. Almdal, Physica B: Condensed Matter 234-236, 260 (1997).

[145] D. Schwahn and L. Willner, Macromolecules 35, 239 (2002).

[146] N. Kučerka, M.-P. Nieh, and J. Katsaras, Biochimica et Biophysica Acta 1808, 2761 (2011).

[147] T. Harroun, W. Heller, T. Weiss, L. Yang, and H. Huang, Biophysical Journal 76, 937 (1999).

[148] M. Kranenburg, J.-P. Nicolas, and B. Smit, Physical Chemistry Chemical Physics 6, 4142 (2004).

[149] S. Leekumjorn and A. K. Sum, Biochimica et Biophysica Acta 1768, 354 (2007).

[150] E. Kurtisovski, N. Taulier, R. Ober, M. Waks, and W. Urbach, Physical Review Letters 98, 258103 (2007). 
[151] W. Helfrich, Zeitschrift für Naturforschung 33A, 305 (1978).

[152] R. Dimova, Advances in Colloid and Interface Science 208, 225 (2014).

[153] R. S. Gracià, N. Bezlyepkina, R. L. Knorr, R. Lipowsky, and R. Dimova, Soft Matter 6, 1472 (2010).

[154] S. Joubaud, A. Petrosyan, S. Ciliberto, and N. B. Garnier, Physical Review Letters 100, 180601 (2008).

[155] S. Leikin, M. Kozlov, N. Fuller, and R. Rand, Biophysical Journal 71, 2623 (1996).

[156] N. Fuller, C. R. Benatti, and R. P. Rand, Biophysical Journal 85, 1667 (2003).

[157] D. Mannock, R. Lewis, R. McElhaney, P. Harper, D. Turner, and S. Gruner, European Biophysics Journal 30, 537 (2001).

[158] J. M. Seddon and R. H. Templer, Polymorphism of Lipid-Water Systems (Elsevier SPC, 1995), pp. 97-160.

[159] C. V. Kulkarni, W. Wachter, G. Iglesias-Salto, S. Engelskirchen, and S. Ahualli, Physical Chemistry Chemical Physics 13, 3004 (2011).

[160] M. M. Kozlov, in Methods in Membrane Lipids, edited by A. M. Dopico (Humana Press, 2007), vol. 400 of Methods in Molecular Biology, pp. 355366.

[161] G. Palazzo, Soft Matter 9, 10668 (2013).

[162] S.-H. Tung, Y.-E. Huang, and S. R. Raghavan, Journal of the American Chemical Society 128, 5751 (2006). 


\section{Curriculum Vitae}

\section{Personal Data}

Name Israel Abraham Barragán Vidal

Date of birth 29.07.1984

Place of birth Distrito Federal, México

Nationality Mexican

Address Weender Landstraße 54, 37075, Göttingen

\section{Education}

10.2001 - 05.2004 Antonio Caso High School (ENP 6), Distrito Federal (México)

08.2005-06.2009 Bachelor of Physics, Faculty of Sciences, National Autonomous University of México, Distrito Federal (México)

08.2009 - 11.2011 Master of Sciences (Physics), Faculty of Sciences, National Autonomous University of México, Distrito Federal (México)

07.2012 - PhD student in the group of Prof. Dr. Marcus Müller, Institute for Theoretical Physics, Georg-August-Universität, Göttingen 